\title{
WestVirginiaUniversity
}

THE RESEARCH REPOSITORY @ WVU

Graduate Theses, Dissertations, and Problem Reports

2009

\section{Hamiltonian line graphs and claw -free graphs}

Huiya Yan

West Virginia University

Follow this and additional works at: https://researchrepository.wvu.edu/etd

\section{Recommended Citation}

Yan, Huiya, "Hamiltonian line graphs and claw -free graphs" (2009). Graduate Theses, Dissertations, and Problem Reports. 2873.

https://researchrepository.wvu.edu/etd/2873

This Dissertation is protected by copyright and/or related rights. It has been brought to you by the The Research Repository @ WVU with permission from the rights-holder(s). You are free to use this Dissertation in any way that is permitted by the copyright and related rights legislation that applies to your use. For other uses you must obtain permission from the rights-holder(s) directly, unless additional rights are indicated by a Creative Commons license in the record and/ or on the work itself. This Dissertation has been accepted for inclusion in WVU Graduate Theses, Dissertations, and Problem Reports collection by an authorized administrator of The Research Repository @ WVU.

For more information, please contact researchrepository@mail.wvu.edu. 


\title{
Hamiltonian Line Graphs and Claw-free Graphs
}

\author{
Huiya Yan \\ Dissertation submitted to the \\ Eberly College of Arts and Sciences \\ at West Virginia University \\ in partial fulfillment of the requirements \\ for the degree of \\ Doctor of Philosophy \\ in \\ Mathematics \\ Hong-Jian Lai, Ph.D., Chair \\ Elaine M Eschen, Ph.D. \\ John Goldwasser, Ph.D. \\ Jerzy Wojciechowski, Ph.D. \\ Cun-Quan Zhang, Ph.D. \\ Department of Mathematics \\ Morgantown, West Virginia \\ 2009
}

Keywords: Line graph, Claw-free graph, Hamiltonicity,

Collapsible, Reduction, Supereulerian graph, 2-factor

Copyright 2009 Huiya Yan 


\section{ABSTRACT \\ Hamiltonian Line Graphs and Claw-free Graphs}

\section{Huiya Yan}

The research of my dissertation was motivated by the conjecture of Thomassen that every 4-connected line graph is hamiltonian and by the conjecture of Matthews and Sumner that every 4-connected claw-free graph is hamiltonian. Towards the hamiltonian line graph problem, we proved that every 3 -connected claw-free $Z_{8}$-free graph is hamiltonian, where $Z_{8}$ is obtained by identifying one end-vertex of $P_{9}$ with one vertex of a triangle; let $h c(G)$ denote the least integer $m$ such that the iterated line graph $L^{m}(G)$ is Hamiltonconnected, we showed that $k-1 \leq h c(G) \leq \max \{\operatorname{diam}(G), k-1\}$, where $k$ is the length of a longest path whose internal vertices, if any, have degree 2 in $G$, and also showed that $\kappa^{3}(G) \leq h c(G) \leq \kappa^{3}(G)+2$ where $\kappa^{3}(G)$ is the least integer $m$ such that $L^{m}(G)$ is 3-connected; moreover, $h c(G) \leq|V(G)|-\Delta(G)+1$.

Chvátal and Erdös showed that the graph $G$ with connectivity $\kappa(G)$ not less than its independence number $\alpha(G)$ (i.e. $\kappa(G) \geq \alpha(G)$ ) is hamiltonian. In this dissertation, we characterized the 2-connected graph $G$ with $\kappa(G) \geq \alpha(G)-1$.

On the other side, we studied the number of components of a 2-factor in a graph to approach its hamiltonicity. In this dissertation, we gave a bound for the number of components of a 2-factor in the line graph of $G$ if $\max \{d(x), d(y)\} \geq \frac{n-\mu}{p}-1$ holds for any $x y \notin E(G)$ and $|U| \neq 2$, where $U=\left\{v: d(v)<\frac{n-\mu}{p}-1\right\}, p$ is a positive integer and $\mu$ is a nonnegative integer.

As an application of graph theory in electrical engineering, we studied the inverse problems on networks at the end of the dissertation and showed that the conductivities in a tree network can be uniquely determined by measurements at the boundary of the voltages generated by imposed currents. 


\section{Acknowledgements}

First and foremost, I am most indebted to my supervisor, Dr. Hong-Jian Lai, for his continued encouragement and support over these last few years. It is a pleasure to work under his supervision. Without him, this paper could not have come about.

I would like to take this opportunity to thank Dr. Liming Xiong at Beijing Institute of Technology, who brought me to the research field of graph theory.

I would also like to thank my other committee members: Dr. Elaine Eschen, Dr. John Goldwasser, Dr. Jerzy Wojciechowski, and Dr. Cun-Quan Zhang, for their help during my studies. Special thanks to Dr. Weifu Fang at Wright State University for introducing Inverse Problems to me.

And finally, I would like to thank the Department of Mathematics and Eberly College of Arts and Sciences at West Virginia University for providing me with an excellent study environment and support during my study as a graduate student. 


\section{DEDICATION}

To

my father Tao Yan, my mother Xueqin Wang

and

my brother Huijun 


\section{Contents}

1 Preliminaries 1

1.1 Notation and Terminology . . . . . . . . . . . . . . . . 1

1.2 Catlin's Reduction Method . . . . . . . . . . . . . . . . . 5

1.3 Main Results . . . . . . . . . . . . . . . . . . . 8

2 Hamiltonian claw-free graphs $\quad 10$

2.1 Closure of claw-free graphs . . . . . . . . . . . . . . . . . . . . . . 10

2.2 Introduction . . . . . . . . . . . . . . . . . . . 11

2.3 Proof of Theorem $2.2 .4 \ldots \ldots \ldots \ldots \ldots$

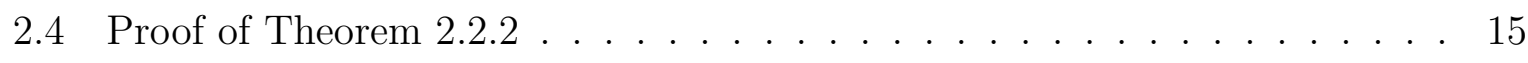

2.5 Concluding remark . . . . . . . . . . . . . . . . . . . . 20

3 Hamilton-connected line graphs $\quad 22$

3.1 Introduction . . . . . . . . . . . . . . . . 22

3.2 Hamilton-connected index and diameter . . . . . . . . . . . . . . 24 
3.3 Hamilton-connected index and connectivity . . . . . . . . . . . . . 32

3.4 Hamilton-connected index and degree . . . . . . . . . . . . . . . . 34

4 The Chvátal-Erdös condition for supereulerian graphs 38

4.1 Introduction . . . . . . . . . . . . . . . . 38

4.2 Proof of Theorem $4.1 .3 \ldots \ldots \ldots \ldots \ldots$. . . . . . . . . . . . . . . 42

4.3 Proof of Theorem $4.1 .6 \ldots \ldots \ldots \ldots$. . . . . . . . . . . . . . 47

$5 \quad$ 2-factors in Line Graphs $\quad 53$

5.1 Introduction . . . . . . . . . . . . . . . 53

5.2 Proof of Theorem $5.1 .7 \ldots \ldots \ldots \ldots \ldots \ldots$

5.3 Corollaries . . . . . . . . . . . . . . . . . . . . . . 58

$\begin{array}{lll}6 & \text { Inverse problems on Graphs } & 61\end{array}$

6.1 Introduction . . . . . . . . . . . . . . . . 61

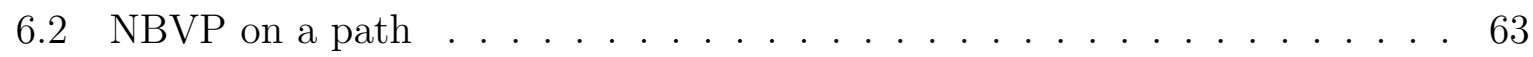

6.3 Inverse problems on electrical networks . . . . . . . . . . . . 65

6.3.1 Network on a complete graph . . . . . . . . . . . . . 65

6.3.2 Network on a tree . . . . . . . . . . . . . . . . 69 


\section{Chapter 1}

\section{Preliminaries}

\subsection{Notation and Terminology}

We use [3] for terminology and notations not defined here. All graphs in this thesis are finite and undirected with no loops or multiple edges. Let $G$ be a graph. We use $V(G)$ and $E(G)$ to denote the set of vertices and the set of edges of $G$, respectively. Two vertices $u, v$ are adjacent if $u v \in E(G)$. If any two vertices are adjacent in $G$, then $G$ is called a complete graph. The independence number $\alpha(G)$ of a graph $G$ is the cardinality of the largest independent vertex subset.

The number of edges incident with a vertex $v \in V(G)$ is called the degree of $v$ in $G$, and is denoted by $d_{G}(v)$ or $d(v)$. We use $\Delta(G)$ and $\delta(G)$ to denote the maximum and minimum degree of $G$, respectively. A graph with at least two vertices is called a nontrivial graph. For an integer $k>0$, a $k$-cycle, denoted by $C_{k}$, is a connected graph with $k$ vertices and in which each vertex has degree 2 ; similarly $P_{k}$ denotes a path with $k$ vertices. For any two vertices $v_{1}, v_{2} \in V(G)$, the distance $d\left(v_{1}, v_{2}\right)$ between $v_{1}$ and $v_{2}$ is defined as the length of the shortest $\left(v_{1}, v_{2}\right)$-path in $G$. The circumference $c(G)$ of a graph $G$ is the length of the longest cycle (a $c(G)$-cycle) in $G$. The girth $g(G)$ of $G$ is the length of the shortest cycle (a $g(G)$-cycle) in $G$. The diameter of $G$ 
is $\operatorname{diam}(G)=\max _{v \in V(G)}\{\max \{d(v, w) \mid w \in V(G)\}\}$. A vertex cut of $G$ is a subset $V^{\prime}$ of $V(G)$ such that $G-V^{\prime}$ is disconnected. A $k$-vertex cut is a vertex cut of $k$ elements. A complete graph has no vertex cut; in fact, the only graphs that do not have vertex cuts are those that contain complete graphs as spanning subgraphs. If $G$ has at least one pair of distinct nonadjacent vertices, the connectivity $\kappa(G)$ of $G$ is the minimum $k$ for which $G$ has a $k$-vertex cut; otherwise, we define $\kappa(G)$ to be $|V(G)|-1$. $G$ is said to be $k$-connected if $\kappa(G) \geq k$. Similarly, we define the edge-connectivity $\kappa^{\prime}(G)$ of $G$ to be the minimum $k$ for which $G$ has a $k$-edge cut. $G$ is said to be $k$-edge-connected if $\kappa^{\prime}(G) \geq k$. A connected graph that has no cut vertices is called a block. Every block with at least three vertices is 2-connected. A block of a graph is a subgraph that is a block and is maximal with respect to this property. An edge $e=u v$ is called a pendant edge of graph $G$ if either $d_{G}(u)=1$ or $d_{G}(v)=1$. For a vertex or an edge subset $X$ of $G, G[X]$ denotes the subgraph of $G$ induced by $X$.

For a graph $G$ and for $v \in V(G)$, the neighborhood $N_{G}(v)$ denotes the set of all vertices adjacent to $v$ in $G$. A vertex $v \in V(G)$ is called a locally connected vertex if $G\left[N_{G}(v)\right]$ is connected. A graph $G$ is locally connected if every vertex of $G$ is locally connected.

For a graph $G$ and each $i=0,1,2, \cdots$, we let $D_{i}(G)=\left\{v \in V(G) \mid d_{G}(v)=i\right\}$. For $H \subseteq G$ and $x \in V(G)$, we let $d_{H}(x)=\left|N_{H}(x)\right|$ and if $A \subseteq V(G)$, we let $G-A=$ $G[V(G)-A]$. When $A=\{v\}$, we use $G-v$ for $G-\{v\}$.

An edge cut $X$ of $G$ is essential if at least two components of $G-X$ are nontrivial. A graph $G$ is essentially $k$-edge-connected if $|E(G)| \geq k+1$ and if for every $E_{0} \subseteq E(G)$ with $\left|E_{0}\right|<k, G-E_{0}$ has exactly one component $H$ with $E(H) \neq \emptyset$.

Let $X \subseteq E(G)$. The contraction $G / X$ is the graph obtained from $G$ by identifying the two ends of each edge in $X$ and then deleting the resulting loops. For convenience, we use $G / e$ for $G /\{e\}$ and $G / \emptyset=G$; and if $H$ is a subgraph of $G$, we write $G / H$ for $G / E(H)$. Note that even if $G$ is a simple graph, contracting some edges of $G$ may result in a graph with multiple edges. If $K$ is a connected subgraph of $G$, and if $v_{K}$ is the vertex in $G / K$ onto which $K$ is contracted, then $K$ is called the pre-image of $v_{K}$, and is denoted by $P I\left(v_{K}\right)$. A vertex $v$ in a contraction of $G$ is a nontrivial vertex if $P I(v)$ has at least 
one edge.

The line graph of a graph $G$, denoted by $L(G)$, has $E(G)$ as its vertex set, where two vertices in $L(G)$ are adjacent if and only if the corresponding two edges in $G$ share one common end-vertex. The iterated line graph is defined recursively by $L^{0}(G)=G$ and $L^{k+1}(G)=L\left(L^{k}(G)\right)(k \in \mathbf{N}$, where $\mathbf{N}$ stands for the set of all natural numbers). Chartrand [14] showed that if $G$ is a connected graph that is not a path, then for some integer $k>0, L^{k}(G)$ is hamiltonian.

Proposition 1.1.1 Let $G$ be a nontrivial simple graph. Then $L(G)$ is complete if and only if $G$ is a $K_{3}$ or a $K_{1, n}$ for an integer $n \geq 1$.

Proof. The line graph $L(G)$ is complete if and only if any two edges in $G$ are incident. If $|E(G)|=1, G=K_{2}=K_{1,1}$; if $|E(G)|=2, G=P_{2}=K_{1,2}$; if $|E(G)|=3, G=K_{3}$ or $G=K_{1,3} ;$ if $|E(G)| \geq 4, G=K_{1, n}$.

A subgraph $H$ of a graph $G$ is dominating if $E(G-V(H))=\emptyset$. Let $O(G)$ denote the set of odd degree vertices of $G$. A graph $G$ is eulerian if $O(G)=\emptyset$ and $G$ is connected. Note that the graph $K_{1}$ is also eulerian. A spanning closed trail of $G$ is also called a spanning eulerian subgraph of $G$. A graph is called supereulerian if it contains a spanning eulerian subgraph. If a closed trail $C$ of $G$ satisfies $E(G-V(C))=\emptyset$, then $C$ is called a dominating eulerian subgraph. A spanning cycle of $G$ is a Hamilton cycle of $G$. If $G$ has a Hamilton cycle, then $G$ is hamiltonian.

From the following theorem we can see that there is a close relationship between dominating eulerian subgraphs in $G$ and Hamilton cycles in its line graph $L(G)$.

Theorem 1.1.2 (Harary and Nash-Williams, [29]) Let $G$ be a graph with $|E(G)| \geq 3$. Then $L(G)$ is hamiltonian if and only if $G$ has a dominating eulerian subgraph.

Let $G$ be a graph such that $\kappa(L(G)) \geq 3$ and $L(G)$ is not complete. The core of the graph $G$, denoted by $G_{0}$, is obtained by contracting all pendant edges and contracting exactly one edge $x y$ or $y z$ for each path $P=x y z$ in $G$ with $d_{G}(y)=2$, where $d(x), d(z)>2$ 
since $\kappa(L(G)) \geq 3$. The remaining edge of $P$ will be referred as a nontrivial edge in the contraction. Shao [44] proved Theorem 1.1.3 (a)-(c). In a similar way as Theorem 1.1.3 (c), one can prove Theorem 1.1.3 (d).

Theorem 1.1.3 (Shao, [44]) Let $G_{0}$ be the core of graph $G$, then each of the following holds:

(a) $G_{0}$ is nontrivial and $\delta\left(G_{0}\right) \geq \kappa^{\prime}\left(G_{0}\right) \geq 3$;

(b) $G_{0}$ is well defined;

(c) If $G_{0}$ has a spanning eulerian subgraph, then $G$ has a dominating eulerian subgraph;

(d) If $G_{0}$ has a dominating eulerian subgraph containing all nontrivial vertices and both endvertices of each nontrivial edges, then $G$ has a dominating eulerian subgraph.

A graph $G$ is Hamilton-connected if for any two vertices $u, v \in V(G)(u \neq v)$, there exists a $(u, v)$-path containing all vertices of $G$.

We view a trail of $G$ as a vertex-edge alternating sequence

$$
v_{0}, e_{1}, v_{1}, e_{2}, \cdots, e_{k}, v_{k}
$$

such that all the $e_{i}$ 's are distinct and such that for each $i=1,2, \cdots, k, e_{i}$ is incident with both $v_{i-1}$ and $v_{i}$. All the vertices in $\left\{v_{1}, v_{2}, \cdots, v_{k-1}\right\}$ are internal vertices of the trail in (1.1). For edges $e^{\prime}, e^{\prime \prime} \in E(G)$, an $\left(e^{\prime}, e^{\prime \prime}\right)$-trail of $G$ is a trail of $G$ whose first edge is $e^{\prime}$ and whose last edge is $e^{\prime \prime}$. (Thus the trail in (1.1) is an $\left(e_{1}, e_{k}\right)$-trail). A dominating $\left(e^{\prime}, e^{\prime \prime}\right)$-trail of $G$ is an $\left(e^{\prime}, e^{\prime \prime}\right)$-trail $T$ of $G$ such that every edge of $G$ is incident with an internal vertex of $T$; and a spanning $\left(e^{\prime}, e^{\prime \prime}\right)$-trail of $G$ is a dominating $\left(e^{\prime}, e^{\prime \prime}\right)$-trail $T$ of $G$ such that $V(T)=V(G)$.

Using a similar argument in the proof of Theorem 1.1.2, one can obtain a theorem for Hamilton-connected line graphs. 
Theorem 1.1.4 Let $G$ be a graph with $|E(G)| \geq 3$. Then $L(G)$ is Hamilton-connected if and only if for any pair of edges $e_{1}, e_{2} \in E(G), G$ has a dominating $\left(e_{1}, e_{2}\right)$-trail.

For a graph $G$, an induced subgraph $H$ isomorphic to $K_{1,3}$ is called a claw of $G$, and the only vertex of degree 3 of $H$ is the center of the claw (see Figure 1.1). A graph $G$ is claw-free if it does not contain a claw as an induced subgraph.

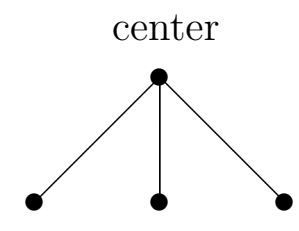

a claw

Figure 1.1

\subsection{Catlin's Reduction Method}

In this section we introduce Catlin's reduction method which is a powerful tool needed in the proofs of a lot of theorems in the dissertation.

A graph $G$ is collapsible if for any even subset $X$ of $V(G), G$ has a spanning connected subgraph $R_{X}$ of $G$ such that $O\left(R_{X}\right)=X$. Catlin [7] showed that every graph $G$ has a unique subgraph $H$ each of whose components is a maximal collapsible subgraph of $G$. The contraction $G / H$ is the reduction of $G$. A graph $G$ is reduced if $G$ has no nontrivial collapsible subgraphs; or equivalently, if $G$ equals the reduction of $G$. We summarize some results on Catlin's reduction method and other related facts below.

Theorem 1.2.1 Let $G$ be a graph and let $H$ be a collapsible subgraph of $G$. Let $v_{H}$ denote the vertex onto which $H$ is contracted in $G / H$. Each of the following holds.

(i) (Catlin, Theorem 3 of [7]) $G$ is collapsible if and only if $G / H$ is collapsible. In particular, $G$ is collapsible if and only if the reduction of $G$ is $K_{1}$. 
(ii) (Catlin, Theorem 8 of [7]) 2-cycles and 3-cycles are collapsible.

(iii) If $G$ is collapsible, then for any pair of vertices $u, v \in V(G), G$ has a spanning $(u, v)$ trail.

(iv) For vertices $u, v \in V(G / H)-\left\{v_{H}\right\}$, if $G / H$ has a spanning $(u, v)$-trail, then $G$ has a spanning $(u, v)$-trail.

(v)(Catlin, Theorem 5 of [7])Any subgraph of a reduced graph is reduced.

(vi) If $G$ is collapsible, and if $e \in E(G)$, then $G / e$ is also collapsible.

Proof (iii) Let $X=\{u, v\}$. Then $|X| \equiv 0(\bmod 2)$, and a spanning connected subgraph $R_{X}=X$ of $G$ with $O\left(R_{X}\right)=\{u, v\}$ is a spanning $(u, v)$-trail.

(iv) Let $\Gamma^{\prime}$ be a spanning $(u, v)$-trail of $G / H$ and let

$$
X=\left\{w \in V(H): w \text { is incident with an odd number of edges in } \Gamma^{\prime}\right\} .
$$

Since $v_{H}$ has even degree in $\Gamma^{\prime},|X| \equiv 0(\bmod 2)$. Let $R_{X}^{\prime}$ be a spanning connected subgraph of $H$ with $O\left(R_{X}^{\prime}\right)=X$. Then $\Gamma=G\left[E\left(\Gamma^{\prime}\right) \cup E\left(R_{X}^{\prime}\right)\right]$ is a spanning $(u, v)$-trail in $G$.

(vi) follows by the definition of collapsible graphs.

Let $\tau(G)$ denote the maximum number of edge-disjoint spanning trees of $G$. Catlin showed the relationship between $\tau(G)$ and the edge-connectivity $\kappa^{\prime}(G)$. Part (ii) of the next theorem is an observation made in [9] and in [11].

Theorem 1.2.2 (Catlin, [7]) Let $G$ be a connected graph and let $H$ be a collapsible subgraph of $G$. Then each of the following holds:

(i) $G$ is reduced if and only if $G$ has no nontrivial collapsible subgraphs;

(ii) $g\left(G^{\prime}\right) \geq 4$ and $\delta\left(G^{\prime}\right) \leq 3$;

(iii) $G$ is supereulerian if and only if $G^{\prime}$ is supereulerian;

(iv) If $G$ is reduced, then $G$ is a simple graph with $\delta(G) \leq 3$ and with either $G \in$ $\left\{K_{1}, K_{2}\right\}$, or $|E(G)| \leq 2|V(G)|-4$. 
Theorem 1.2.3 (Lai, [36]) Let $G$ be a 2-connected graph with $\delta(G) \geq 3$. If every edge of $G$ is in a cycle of length at most 4 , then $G$ is collapsible.

It is known that all complete graphs of order at least 3 are collapsible and any cycle of length at least 4 is not collapsible. If $G$ contains a 4-cycle $C=u v z w u$ with a partition $\pi=\langle\{u, z\},\{v, w\}\rangle$, then we can follow Catlin [6] and define $G / \pi(C)$ to be the graph obtained from $G-E(C)$ by identifying $u$ and $z$ to form a new vertex $x$, identifying $v$ and $w$ to form a new vertex $y$, and adding an edge $e_{\pi}=x y$.

Theorem 1.2.4 (Catlin, [6]) Let $G$ be a graph containing a 4-cycle $C$ and let $G / \pi(C)$ be defined as above. Each of the following holds:

(a) If $G / \pi(C)$ is collapsible, then $G$ is collapsible;

(b) If $G / \pi(C)$ has a spanning eulerian subgraph, then $G$ has a spanning eulerian subgraph, i.e., if $G / \pi(C)$ is supereulerian, then $G$ is supereulerian.

Theorem 1.2.5 Let $G$ be a graph, $H$ be a subgraph of $G$, and $k>0$ be an integer.

(i) (Catlin, [5]) $\kappa^{\prime}(G) \geq 2 k$ if and only if for any edge subset $X \subseteq E(G)$ with $|X| \leq k$, $\tau(G-X) \geq k$.

(ii) If $\tau(H) \geq k$ and if $\tau(G / H) \geq k$, then $\tau(G) \geq k$.

Theorem 1.2.6 (Catlin and Lai, Theorem 4 of [12]) Let $G$ be a graph with $\tau(G) \geq 2$ and let $e^{\prime}, e^{\prime \prime} \in E(G)$. Then $G$ has a spanning $\left(e^{\prime}, e^{\prime \prime}\right)$-trail if and only if $\left\{e^{\prime}, e^{\prime \prime}\right\}$ is not an essential edge cut of $G$.

We define $F(G)$ to be the minimum number of additional edges that must be added to $G$ such that the resulting graph has two edge-disjoint spanning trees.

Theorem 1.2.7 Let $G$ be a graph. 
(i) (Catlin, Han and Lai, Lemma 2.3 of [10]) If for any $H \subset G$ with $|V(H)|<|V(G)|$, $H$ is reduced, and if $|V(G)| \geq 3$, then $F(G)=2|V(G)|-|E(G)|-2$.

(ii) (Catlin, Theorem 7 of [7]) If $F(G) \leq 1$, then $G$ is collapsible if and only if $\kappa^{\prime}(G) \geq 2$.

(iii) (Catlin, Han and Lai, Theorem 1.3 of [10]) Let $G$ be a connected graph and $t$ an integer. If $F(G) \leq 2$, then $G$ is collapsible if and only if $G$ cannot be contracted to a member in $\left\{K_{2}\right\} \cup\left\{K_{2, t}: t \geq 1\right\}$.

\subsection{Main Results}

In the coming several chapters, we will present the following main results.

(1) Every 3-edge-connected graph with the circumference at most 8 is supereulerian, which is then applied to show that a 3 -connected claw-free graph without $Z_{8}$ as an induced subgraph is hamiltonian, where $Z_{8}$ denotes the graph derived from identifying one endvertex of $P_{9}$ (a path with 9 vertices) with one vertex of a triangle. The above two results are both best possible in a sense that the number 8 can not be replaced by 9 and they also extend former results by Brousek, Ryjáček and Favaron in [Discrete Math. 196(1999)2950] and by Łuczak and Pfender in [J. Graph Theory, 47(2004)111-121].

(2) Let $h c(G)$ be the least integer $m$ such that the iterated line graph $L^{m}(G)$ is Hamilton-connected. Let $\operatorname{diam}(G)$ be the diameter of $G$ and $k$ be the length of a longest path whose internal vertices, if any, have degree 2 in $G$. In this dissertation, we show that $k-1 \leq h c(G) \leq \max \{\operatorname{diam}(G), k-1\}$. We also show that $\kappa^{3}(G) \leq h c(G) \leq \kappa^{3}(G)+2$ where $\kappa^{3}(G)$ is the least integer $m$ such that $L^{m}(G)$ is 3-connected. Finally we prove that $h c(G) \leq|V(G)|-\Delta(G)+1$.

(3) A classical result of Chvátal and Erdös says that the graph $G$ with connectivity $\kappa(G)$ not less than its independence number $\alpha(G)$ (i.e. $\kappa(G) \geq \alpha(G)$ ) is hamiltonian. In this thesis, we show that the 2 -connected graph $G$ with $\kappa(G) \geq \alpha(G)-1$ is one of the following: supereulerian, the Petersen graph, the 2-connected graph with three vertices of 
degree two obtained from $K_{2,3}$ by replacing a vertex of degree three with a triangle, one of the 2-connected graphs obtained from $K_{2,3}$ by replacing a vertex of degree two with a complete graph of order at least three and by replacing at most one branch of length two in the resulting graph with a branch of length three, or one of the graphs obtained from $K_{2,3}$ by replacing at most two branches of $K_{2,3}$ with a branch of length three. We also show that the hamiltonian index of the simple 2-connected graph $G$ with $\kappa(G) \geq \alpha(G)-t$ is at most $\left\lfloor\frac{2 t+2}{3}\right\rfloor$ for every nonnegative integer $t$. The upper bound is sharp.

(4) A lot of results on the components of a 2 -factor in $G$ have appeared by studying the conditions on the minimum degree of the graph $G$. In this thesis we avoid studying the minimum degree and get the following: if $\max \{d(x), d(y)\} \geq \frac{n-\mu}{p}-1$ holds for any $x y \notin E(G)$ and $|U| \neq 2$, where $U=\left\{v: d(v)<\frac{n-\mu}{p}-1\right\}, p$ is a positive integer and $\mu$ is a nonnegative integer, then for $n$ sufficiently large relative to $p$ and $\mu, L(G)$ has a 2 -factor with at most $p+1$ components. Moreover, $L(G)$ has a 2 -factor with at most $p$ components if $|U| \leq 1$. Especially, it extends a result of [41] saying that if $\delta(G) \geq \frac{n}{p}-1$, i.e., $U=\emptyset$, then $L(G)$ has a 2-factor with at most $p$ components. We also show the graphs above are $(p+2)$-supereulerian, i.e., have a spanning even subgraph with at most $p+2$ components. All results are best possible.

(5) As an application in electrical engineering, we will show that the conductivities in a tree network can be uniquely determined by measurements at the boundary of the voltages generated by imposed currents. Moreover, the solvability of inverse problem related to NBVP (Neumann Boundary Value Problem) on a path network is proved. The Dirichletto-Neumann matrix of the network on a complete graph is partially characterized. 


\section{Chapter 2}

\section{Hamiltonian claw-free graphs}

\subsection{Closure of claw-free graphs}

Ryjáček [42] introduced the closure of a graph, which works well in the class of claw-free graphs. For $x \in V(G)$, the graph $G_{x}^{\prime}$ obtained from $G$ by adding the edges $\{y z: y, z \in$ $N(x) \& y z \notin E(G)\}$ is called the local completion of $G$ at $x$. The closure of a claw-free graph $G$, denoted by $\operatorname{cl}(G)$, is obtained from $G$ by recursively performing local completions at any locally connected vertex with non-complete neighborhood, as long as it is possible. The following theorem translates claw-free graphs to line graphs when we consider the hamiltonicity of claw-free graphs.

Theorem 2.1.1 (Ryjáček, [42]) Let $G$ be a claw-free graph. Then

(a) $\operatorname{cl}(G)$ is uniquely determined;

(b) $\operatorname{cl}(G)$ is the line graph of a triangle-free graph;

(c) $G$ is hamiltonian if and only if $\operatorname{cl}(G)$ is hamiltonian.

Theorem 2.1.2 (Brousek, Ryjáček and Favaron, [4]) Let $G$ be a claw-free graph. Then 
(a) If $G$ is $Z_{k}$-free, then $\operatorname{cl}(G)$ is also $Z_{k}$-free for any integer $k \geq 1$;

(b) If $G$ is $P_{i}$-free, then $\mathrm{cl}(G)$ is also $P_{i}$-free for any integer $i \geq 3$.

There are two famous conjectures in the fields of Hamiltonian line graphs and Hamiltonian claw-free graphs.

Conjecture 2.1.3 (Thomassen, [45]) Every 4-connected line graph is Hamiltonian.

Conjecture 2.1.4 (Matthews and Sumner, [39]) Every 4-connected claw-free graph is Hamiltonian.

The following theorem unified the two conjectures above.

Theorem 2.1.5 (Ryjáček, [42]) Conjecture 2.1.3 and Conjecture 2.1.4 are equivalent.

\subsection{Introduction}

We use $Z_{k}$ to denote a graph obtained from the disjoint union of a $P_{k+1}$ and a 3-cycle $K_{3}$ by identifying one end-vertex of $P_{k+1}$ with one vertex of $K_{3}$. A graph $G$ is $\left\{H_{1}, H_{2}, \cdots H_{s}\right\}$ free if it contains no induced subgraphs that are isomorphic to a copy of $H_{i}$ for any $i$, where $H_{i}$ is connected for any $i$.

In 1999, Brousek, Ryjáček and Favaron proved the following theorem.

Theorem 2.2.1 (Brousek, Ryjáček and Favaron, [4]) Let $G$ be a 3-connected claw-free graph. If $G$ is $Z_{4}$-free, then $G$ is hamiltonian.

To extend Theorem 2.2.1, we have the following theorem as a main result in this chapter. 
Theorem 2.2.2 Let $G$ be a 3-connected simple claw-free graph. If $G$ is $Z_{8}$-free, then $G$ is hamiltonian.

At the end of Section ??, we will give an example of a 3-connected claw-free nonhamiltonian $Z_{9}$-free graph. In this sense, Theorem 2.2.2 is best possible.

Theorem 2.2.2 also has an immediate consequence: every 3-connected claw-free $P_{10^{-}}$ free graph is hamiltonian since a $P_{10}$-free graph must be $Z_{8}$-free. In fact, it is a slightly weaker case of Theorem 2.2.3. In Section 2.5, we shall reprove Theorem 2.2.3 with the help of our results.

Theorem 2.2.3 (Euczak and Pfender, [38]) Every 3-connected $\left\{K_{1,3}, P_{11}\right\}$-free graph is hamiltonian.

In order to prove Theorem 2.2.2, we need the following associate result.

Theorem 2.2.4 Let $G$ be a 3-edge-connected graph. If $c(G) \leq 8$, then $G$ is supereulerian.

The above result is also best possible since the Petersen graph has circumference 9 but it is not supereulerian. More generally, we let $G$ be a 3-edge-connected graph in which every block is a Petersen graph. Since every cycle of $G$ must be inside a block of $G$, and since the longest cycle in the Petersen graph has length 9 , we conclude that $c(G)=9$. On the other hand, a graph is supereulerian if and only if every block of it is supereulerian. Hence such a graph $G$ is not supereulerian. Since the example permits any finite number of blocks, it shows that the bound 8 in Theorem 2.2.4 is best possible even if we allow for a finite number of exceptions.

Obviously a hamiltonian graph is supereulerian, but the reverse is not true. We also have that the line graph of a supereulerian graph is hamiltonian by Theorem 1.1.2.

A sub-path of a path $P$ is a connected subgraph of $P$. If not otherwise stated subscripts of vertices in a cycle $C$ are taken modulo $|V(C)|$. For a cycle $C=u_{1} u_{2} \cdots u_{m} u_{1}$ 
we use $C\left(u_{i}, u_{j}\right)$ to denote the path $u_{i} u_{i+1} \cdots u_{j}$. We denote by $P(u, v)$ the sub-path of $P$ with the first vertex $u$ and the last vertex $v$. For two sets $S$ and $T$, by $S \triangle T$ we denote the symmetric difference of $S$ and $T$.

Theorem 2.2.5 (Chen, [16]) If $G$ is a 3-edge-connected simple graph with at most 13 vertices, then either $G$ is supereulerian or $G$ is contractible to the Petersen graph.

\subsection{Proof of Theorem 2.2.4}

We argue by contradiction, and assume that $G$ is a counter-example to Theorem 2.2.4 with $|V(G)|$ minimized.

If $G$ is not reduced, then let $G^{\prime}$ be the reduction of $G$. Since $G$ is not reduced and $G^{\prime}$ is a contraction of $G,|V(G)|>\left|V\left(G^{\prime}\right)\right|$ and $\kappa^{\prime}\left(G^{\prime}\right) \geq \kappa^{\prime}(G) \geq 3$. Since any cycle of $G^{\prime}$ can be extended to a cycle of $G, c\left(G^{\prime}\right) \leq c(G) \leq 8$. By the minimality of $|V(G)|, G^{\prime}$ is supereulerian. By Theorem 1.2.2 (d), $G$ is supereulerian, contrary to the choice of $G$.

Hence we may assume that $G$ is reduced. If $G$ has a cut-vertex, then by the minimality of $G$, each block of $G$ is supereulerian, and so $G$ is supereulerian, contrary to the choice of $G$. Therefore, we assume that $\kappa(G) \geq 2$ and $G \neq K_{1}$.

By Theorem 1.2.2, $4 \leq g(G) \leq c(G) \leq 8$. If $g(G)=4$, then we can assume that $H$ is a 4 -cycle $x_{1} x_{2} y_{1} y_{2} x_{1}$ in $G$ with a partition $\pi=\left\langle\left\{x_{1}, y_{1}\right\},\left\{x_{2}, y_{2}\right\}\right\rangle$. We obtain the following three facts to show some good properties of $G$.

Claim 1. $\kappa^{\prime}(G / \pi(H)) \geq 3$.

Proof of Claim 1. By way of contradiction, we assume $\kappa^{\prime}(G / \pi(H)) \leq 2$. It suffices to distinguish the following two cases to obtain our required contradiction.

Case 1.1. $\kappa^{\prime}(G / \pi(H))=1$.

Then $G-E(H)$ has two components $G_{1}$ and $G_{2}$ such that $x_{i}, y_{i} \in V\left(G_{i}\right)$. Let $P\left(x_{i}, y_{i}\right)$ be a longest path between $x_{i}$ and $y_{i}$ in $G_{i}$, then $P\left(x_{i}, y_{i}\right)$ has length at least 
three. Suppose, to the contrary, that $P\left(x_{1}, y_{1}\right)$ has length two, say, $P\left(x_{1}, y_{1}\right)=x_{1} w y_{1}$. Since $\kappa^{\prime}(G) \geq 3, G-\left\{w x_{1}, w y_{1}\right\}$ is still connected. So there exist a pair of paths $P\left(w, x_{1}\right)$ (between $w$ and $\left.x_{1}\right)$ and $P\left(w, y_{1}\right)$ (between $w$ and $\left.y_{1}\right)$ in $G-\left\{w x_{1}, w y_{1}\right\}$ such that either $y_{1} \notin V\left(P\left(w, x_{1}\right)\right)$ or $x_{1} \notin V\left(P\left(w, y_{1}\right)\right)$, say $y_{1} \notin V\left(P\left(w, x_{1}\right)\right)$. Then $y_{1} w P\left(w, x_{1}\right)$ is a longer path than $P\left(x_{1}, y_{1}\right)$ in $G_{1}$, a contradiction. We claim that $P\left(x_{i}, y_{i}\right)$ has length exactly 3 since otherwise $P\left(x_{1}, y_{1}\right) y_{1} x_{2} P\left(x_{2}, y_{2}\right) y_{2} x_{1}$ is a cycle of length at least 9 , a contradiction. Let $P\left(x_{i}, y_{i}\right)=x_{i} u_{i} v_{i} y_{i}$. Since $\delta(G) \geq \kappa^{\prime}(G) \geq 3, d\left(v_{1}\right) \geq 3$ and $d\left(u_{1}\right) \geq 3$, and then there are two vertices $s_{1}$ and $t_{1}$ such that $s_{1} u_{1}, t_{1} v_{1} \in E(G)$. Since $g(G)=4$, $s_{1} \neq t_{1}$. By $\kappa(G) \geq 2, u_{2} v_{2}$ and each of $\left\{u_{1} s_{1}, v_{1} t_{1}\right\}$ must be in a cycle of length at most 8. Hence the assumption that $g(G)=4$ forces that $s_{1} y_{1}, t_{1} x_{1} \in E(G)$. But then $s_{1} u_{1} v_{1} t_{1} x_{1} x_{2} u_{2} v_{2} y_{2} y_{1} s_{1}$ is a 10 -cycle, contrary to the assumption that $c(G) \leq 8$.

Case 1.2. $\kappa^{\prime}(G / \pi(H))=2$.

Then $G-E(H)$ has a cut edge $e=z_{1} z_{2}$ and $G-(E(H) \cup\{e\})$ has two components $G_{1}$ and $G_{2}$ such that $x_{i}, y_{i}, z_{i} \in V\left(G_{i}\right)$. Since $\kappa^{\prime}(G) \geq 3$ and $g(G) \geq 4, G_{i}$ has at least five vertices.

Let $W_{i}=\left\{x_{i}, y_{i}, z_{i}\right\}$ and suppose $u_{i}, v_{i} \in V\left(G_{i}\right) \backslash W_{i}$ for $i=1,2$.

If every element in the vertex set $V\left(G_{i}\right) \backslash W_{i}$ has neighbors which are all in $W_{i}$, then $v_{1} z_{1} z_{2} v_{2} y_{2} u_{2} x_{2} x_{1} u_{1} y_{1} v_{1}$ is a 10 -cycle of $G$ since $\kappa^{\prime}(G) \geq 3$, contrary to the assumption that $c(G) \leq 8$.

Suppose only one of $\left\{G_{1}, G_{2}\right\}$, say $G_{1}$, has the property that all vertices in $V\left(G_{1}\right) \backslash W_{1}$ have neighbors only in $W_{1}$, i.e., there is at least one vertex $u_{2}$ of $V\left(G_{2}\right) \backslash W_{2}$ which has a neighbor $v_{2} \notin W_{2}$. Since $\kappa(G) \geq 2$, there is a cycle $C_{1}$ containing two edges $u_{2} v_{2}$ and $z_{1} z_{2}$. The fact that $z_{1} z_{2}$ is a cut edge of $G-E(H)$ implies that $C_{1}$ contains exactly one element of $W_{i} \backslash\left\{z_{i}\right\}$. By the supposition of $G_{1}$, we can take two vertices $u_{1}, v_{1} \in V\left(G_{1}\right) \backslash W_{1}$ such that they are adjacent to every element of $W_{1}$ and hence there is a cycle of length at least 9 , (for the shortest case) say $z_{1} u_{1} x_{1} y_{2} y_{1} x_{2} u_{2} v_{2} z_{2} z_{1}$, in $G\left[V\left(C_{1}\right) \bigcup V(H) \bigcup\left\{u_{1}, v_{1}, u_{2}, v_{2}\right\}\right]$, contrary to the assumption that $c(G) \leq 8$.

It remains the case when there are two edges $u_{1} v_{1}$ and $u_{2} v_{2}$ in $G_{1}$ and $G_{2}$ respectively such that they are not incident with any element of $W_{1} \bigcup W_{2}$. Since $\kappa(G) \geq 2$, there is a 
cycle $C_{1}$ containing two edges $u_{1} v_{1}, u_{2} v_{2}$. Hence $\left|E\left(C_{1}\right)\right|=8$ by $g(G) \leq 8$. Note that $C_{1}$ contains exactly two elements of $W_{i}$. If $z_{1} z_{2} \in E\left(C_{1}\right)$, then $G\left[E\left(C_{1}\right) \triangle E\left(x_{1} x_{2} y_{1} y_{2}\right)\right]$ is a cycle of length at least 9 , a contradiction. Now suppose that $z_{1} z_{2} \notin E\left(C_{1}\right)$. Then there is a cycle $C_{2}$ containing $z_{1} z_{2}$ and $u_{1} v_{1}$. Hence $G\left[V\left(C_{1}\right) \cup V\left(C_{2}\right)\right]$ contains a cycle of length at least 9 , contrary to the assumption that $c(G) \leq 8$. This completes the proof of Claim 1 .

Claim 2. $g(G) \geq 5$.

Proof of Claim 2. Assume, in contrast, that $G$ has a 4-cycle $C$, then by Claim 1, $\kappa^{\prime}(G / \pi(C)) \geq 3$. By the definition of $G / \pi(C)$, any cycle of $G / \pi(C)$ can be (possibly trivially) extended to a cycle of $G$, and so $c(G / \pi(C)) \leq c(G) \leq 8$. By the minimality of $|V(G)|, G / \pi(C)$ is supereulerian. Then by Theorem 1.2.4 (b), $G$ is also supereulerian, contrary to the choice of $G$. This completes the proof of Claim 2.

By Claims 1 and 2, we can assume that $g(G) \geq 5, \kappa^{\prime}(G) \geq 3$ and $\kappa(G) \geq 2$. Hence $\delta(G) \geq \kappa^{\prime}(G) \geq 3$.

Now take a longest path $x_{1} x_{2} \ldots x_{l}$ in $G$ and note that the end vertices have neighbors only on the path. As $\delta(G) \geq 3, x_{1}$ has at least three such neighbors. As $g(G) \geq 5$, $N\left(x_{1}\right)=\left\{x_{2}, x_{5}, x_{8}\right\}$, otherwise there is a cycle of length at least 9 , a contradiction. Using the alternative longest path $x_{4} x_{3} x_{2} x_{1} x_{5} x_{6} \ldots x_{l}$, we get $x_{4} x_{8} \in E(G)$ by the same argument, yielding a $C_{4}=x_{1} x_{5} x_{4} x_{8} x_{1}$, contrary to Claim 2. This completes the proof of Theorem 2.2.4.

\subsection{Proof of Theorem 2.2.2}

By Theorems 2.1.1 and 2.1.2, Theorem 2.2.2 can be equivalently expressed as: If $G$ is a 3-connected $Z_{8}$-free line graph, then $G$ is hamiltonian.

A $Y_{m}$ in $G$ is any subgraph of $G$ isomorphic to the (unique) tree $Y$ on $m+2$ vertices with exactly 3 leaves such that the unique vertex of degree 3 in $Y_{m}$ is adjacent to two of the three leaves, where a leaf of a tree is defined to be a vertex of degree one. Note that 
the tree is shaped like the letter $Y$. Then $L(G)$ is $Z_{8}$-free if and only if $G$ is a connected simple graph without subgraphs isomorphic to $Y_{10}$. To describe a $Y_{m}$, we only list its edges.

Therefore, to show Theorem 2.2.2, it suffices to prove the following theorem.

Theorem 2.4.1 Let $G$ be a connected simple graph without subgraphs isomorphic to $Y_{10}$. If $\kappa(L(G)) \geq 3$, then $L(G)$ is hamiltonian.

Before presenting the proof of Theorem 2.4.1, we need the following two lemmas, which will also be used to reprove Theorem 2.2.3 in the last section.

Lemma 2.4.2 Let $G$ be a reduced graph with $\kappa^{\prime}(G) \geq 3, g(G) \geq 4$ and $c(G) \geq 9$, and let $C=u_{1} u_{2} \cdots u_{c(G)}$ be a longest cycle of $G$. If $G$ contains no subgraphs isomorphic to $Y_{10}$ and $P_{12}$, then any vertex in $V(G) \backslash V(C)$ has at most one neighbor not on $C$ and hence has at least two neighbors on $C$ since $\kappa^{\prime}(G) \geq 3$.

Proof of Lemma 2.4.2. Suppose there is a vertex $w$ in $V(G) \backslash V(C)$ which has at least two neighbors not on $C$. Let $P\left(w, u_{i}\right)$ be a shortest path between $w$ and $C$ with $u_{i} \in V(C)$. Then we can take two neighbors, $x, y$ (say), of $w$, such that $x$ and $y$ are not in $V\left(P\left(w, u_{i}\right)\right) \cup V(C)$ since $d(w) \geq \kappa^{\prime}\left(G_{0}\right) \geq 3$. Hence

$$
\{x w, y w\} \cup E\left(P\left(w, u_{i}\right)\right) \cup E\left(C\left(u_{i}, u_{i+9}\right)\right) \text { is a } Y_{9+\left|E\left(P\left(w, u_{i}\right)\right)\right|} \text {. }
$$

Let $P(x, y, w)$ be a longest path containing each of $\{x, y, w\}$ such that it contains exactly one vertex of $C$ which is the last vertex of this path. Then $P(x, y, w)$ has length at least three by $g(G) \geq 4$. Without loss of generality, we assume $u_{1} \in V(P(x, y, w)) \cap V(C)$. Hence

$$
P(x, y, w) C\left(u_{1}, u_{9}\right) \text { is } P_{9+|E(P(x, y, w))|} \cdot
$$

(2.1) and (2.2) contradict the hypothesis of Lemma 2.4.2. This completes the proof of Lemma 2.4.2. 
In the proof of Lemma 2.4.3, we use the following additional notation. For the reduction $G^{\prime}$ of a connected graph $G$, let

$\Lambda\left(G^{\prime}\right)=\left\{v \in V\left(G^{\prime}\right): v\right.$ is nontrivial or an end-vertex of a nontrivial edge of $\left.G^{\prime}\right\}$.

Lemma 2.4.3 Let $G$ be a connected simple graph without subgraphs isomorphic to $Y_{10}$ or $P_{12}$, and $G_{0}$ be the core of $G$. If $G_{0}$ is the reduced graph with $\kappa^{\prime}\left(G_{0}\right) \geq 3, g\left(G_{0}\right) \geq 4$ and $c\left(G_{0}\right)=9$, then $G_{0}$ has a dominating closed trail $H$ such that $\Lambda\left(G_{0}\right) \subseteq V(H)$.

Proof of Lemma 2.4.3. We argue by contradiction, and assume that $G_{0}$ is a counterexample to Lemma 2.4.3.

Let $C=u_{1} u_{2} \cdots u_{9} u_{1}$ be a 9-cycle that contains as many vertices of $\Lambda\left(G_{0}\right)$ as possible.

We distinguish the following two cases to obtain our desired contradiction.

Case 1. $C$ is not dominating.

We can take an edge $x y \in E\left(G_{0}\right)$ such that $x, y \notin V(C)$. By Lemma 2.4.2 and $d_{G_{0}}(x), d_{G_{0}}(y) \geq 3$, both $x$ and $y$ have at least two neighbors on $C$. Noticing the fact that $4 \leq g\left(G_{0}\right) \leq c\left(G_{0}\right)=9$, we claim that $d_{C}(s, t) \geq 3$ for any pair of vertices $s \in$ $N_{G_{0}}(x) \cap V(C)$ and $t \in N_{G_{0}}(y) \cap V(C)$, where $d_{C}(s, t)$ means the length of the shortest path on $C$ between $s$ and $t$. By this claim and the fact that both $x$ and $y$ have at least two neighbors on $C$ we obtain that there is either a triangle or a cycle of length at least ten, a contradiction.

Case 2. $C$ is a dominating closed trail but it does not contain all elements of $\Lambda\left(G_{0}\right)$.

There exists at least one vertex in $\Lambda\left(G_{0}\right) \backslash V(C)$. Since $C$ is dominating in $G_{0}$, we only need to count the number of vertices not in $C$.

Suppose $\left|V\left(G_{0}\right)\right| \leq 13$, i.e., $\left|V\left(G_{0}\right)-V(C)\right| \leq 4$. Hence by Theorem $2.2 .5, G_{0}$ is supereulerian or the Petersen graph. If $G_{0}$ is supereulerian, then obviously Lemma 2.4.3 holds. So we only need to consider the case when $G_{0}$ is the Petersen graph, then all the ten vertices in $G_{0}$ must be in $\Lambda\left(G_{0}\right)$ since otherwise there is a dominating cycle of length 9 containing all such kind of vertices. Let $x_{1} x_{2} \cdots x_{10}$ be a longest path of $G_{0}$. 
For each $i \in\{1,2, \cdots, 10\}$, if $x_{i}$ is nontrivial, then $x_{i}$ has at least a neighbor $v_{i}$ in the original graph $G$; if $x_{i}$ is one end vertex of a nontrivial edge of $G_{0}$, then there is a path of length two whose internal vertex $v_{i}$ has degree two in the original graph $G$. Then $\left\{x_{2} v_{2}, x_{10} v_{10}\right\} \bigcup E\left(x_{1} x_{2} \cdots x_{10}\right)$ is a $Y_{10}$ and $v_{1} x_{1} x_{2} \cdots x_{10} v_{10}$ is a $P_{12}$ in $G$, which is a contradiction.

It remains the case that $\left|V\left(G_{0}\right)\right| \geq 14$, i.e., there are at least 5 vertices not on $C$. Let $w \in \Lambda\left(G_{0}\right) \backslash V(C)$. Let $w^{\prime}$ be the neighbor of $w$ in the original graph $G$. Since $C$ is dominating in $G_{0}$ and $\kappa^{\prime}\left(G_{0}\right) \geq 3$, there must be a neighbor $u_{1}$ (say) of $w$ on $C$ such that there is a vertex $u_{8}$ (say) of $C$ with distance two from $u_{1}$ on $C$ and $u_{8}$ has a neighbor $v$ (say) not on $C$. Let $w^{\prime}$ be the neighbor of $w$ in the original graph $G$. Then

$$
\left\{v u_{8}, w w^{\prime}, w u_{1}\right\} \bigcup E\left(C\left(u_{1}, u_{9}\right)\right) \text { is a } Y_{10} \text { in } G .
$$

Similarly, there must be a neighbor $u_{1}$ (say) of $w$ on $C$ such that there is a vertex $u_{9}$ (say) of $C$ with distance one from $u_{1}$ on $C$ and $u_{9}$ has a neighbor $v_{9}$ (say) not on $C$. Then

$$
w^{\prime} w u_{1} C\left(u_{1}, u_{9}\right) v_{9} \text { is a } P_{12} .
$$

(2.3) and (2.4) contradict the hypothesis of Lemma 2.4.3, which completes the proof of Lemma 2.4.3.

Now we present the proof of Theorem 2.4.1.

Proof of Theorem 2.4.1. Let $G_{0}$ be the core of $G$. To show $L(G)$ is hamiltonian, by Theorems 1.1.3 and 1.1.2, it suffices to show the following claim.

Claim 3. $G_{0}$ has a dominating closed trail $H$ such that $\Lambda\left(G_{0}\right) \subseteq V(H)$.

Proof of Claim 3. We argue by contradiction, and assume that $G_{0}$ is a counter-example to Claim 3 with $\left|V\left(G_{0}\right)\right|$ minimized.

If $G_{0}$ is not reduced, then let $G_{0}^{\prime}$ be the reduction of $G_{0}$. Hence $\left|V\left(G_{0}\right)\right|>\left|V\left(G_{0}^{\prime}\right)\right|$. By the minimality of $\left|V\left(G_{0}\right)\right|, G_{0}^{\prime}$ has a dominating closed trail $H^{\prime}$ such that $V\left(H^{\prime}\right)$ contains all nontrivial vertices and end-vertices of all nontrivial edges of $G_{0}^{\prime}$. Let $W_{1}$ be the set of all nontrivial vertices obtained by contracting $G_{0}$ to $G_{0}^{\prime}$ and $W_{2}$ be the set of all nontrivial vertices and end-vertices of all nontrivial edges obtained by contracting $G$ to $G_{0}$. Then 
$W_{2}=\Lambda\left(G_{0}\right)$. Note that if $w \in W_{2}$ then there is either a neighbor of $w$ in the original graph $G$ if $w$ is a nontrivial vertex, or a vertex with degree two such that it is in a path containing $w$ if $w$ is an end vertex of a nontrivial edge of $G_{0}$.

For any vertex $v^{\prime}$ in $W_{1}$, we have that $P I\left(v^{\prime}\right)$ is collapsible. Let

$$
S=\left\{z \in V\left(P I\left(v^{\prime}\right)\right): z \text { is incident with an odd number of edges in } E\left(H^{\prime}\right)\right\} .
$$

Then $|S| \equiv 0(\bmod 2)$ since $H^{\prime}$ is eulerian. Hence $\left|S \triangle O\left(P I\left(v^{\prime}\right)\right)\right|$ is even. Since $P I\left(v^{\prime}\right)$ is collapsible, there exists a spanning connected subgraph $T_{v^{\prime}} \subseteq P I\left(v^{\prime}\right)$ such that $O\left(T_{v^{\prime}}\right)=$ $S \triangle O\left(P I\left(v^{\prime}\right)\right)$. Let $H=G\left[E\left(H^{\prime}\right) \bigcup\left\{\bigcup E\left(T_{v^{\prime}}\right): v^{\prime} \in W_{1}\right\}\right]$. Then $H$ is a dominating closed trail of $G_{0}$ that contains every element of $W_{2}$, a contradiction.

Hence we suppose that $G_{0}$ is reduced. By Theorem 1.1.3, we have $\delta\left(G_{0}\right) \geq \kappa^{\prime}\left(G_{0}\right) \geq 3$. By Lemma 2.4.3, it suffices to consider the case that $c\left(G_{0}\right) \geq 10$ since otherwise $G_{0}$ itself is supereulerian by Theorem 2.2.4 which contradicts our assumption.

Let $C=u_{1} u_{2} \cdots u_{c\left(G_{0}\right)} u_{1}$ be a longest cycle of $G_{0}$. If $c\left(G_{0}\right) \geq 11$, then $V\left(G_{0}\right) \backslash$ $V(C) \neq \emptyset$ since otherwise $C$ is a spanning closed trail of $G_{0}$, a contradiction. Hence we can take a vertex $u \in V\left(G_{0}\right) \backslash V(C)$ such that $u$ is adjacent to one vertex of $C$, say $u_{1}$, then $\left\{u_{1} u, u_{1} u_{c\left(G_{0}\right)}\right\} \bigcup E\left(C\left(u_{1}, u_{10}\right)\right)$ is a $Y_{10}$, which contradicts the assumption of Theorem 2.4.1.

The only case left is $c\left(G_{0}\right)=10$. Theorem 2.2 .5 implies $\left|V\left(G_{0}\right)\right| \geq 14$, i.e., $\mid V\left(G_{0}\right) \backslash$ $V(C) \mid \geq 4$. If there are two vertices $x, y$ of $V\left(G_{0}\right) \backslash V(C)$ such that they have the same neighbor $u_{1}$ (say) on $C$, then $\left\{x u_{1}, y u_{1}\right\} \cup E\left(u_{1}, u_{10}\right)$ is a $Y_{10}$, a contradiction; in the case that every vertex of $V\left(G_{0}\right) \backslash V(C)$ has a different neighbor on $C$ than others, by the fact $\left|V\left(G_{0}\right) \backslash V(C)\right| \geq 4$ and Lemma 2.4.2, we can find two vertices $x, y$ of $V\left(G_{0}\right) \backslash V(C)$ such that they have neighbors $u_{2}$ and $u_{10}$ (say) with distance two on $C$ respectively since $\delta\left(G_{0}\right) \geq \kappa^{\prime}\left(G_{0}\right) \geq 3$ and $G_{0}$ is triangle-free. Then $\left\{x u_{2}, y u_{10}\right\} \cup E\left(C\left(u_{1}, u_{10}\right)\right)$ is a $Y_{10}$, a contradiction.

This completes the proof of Claim 3 and (hence) of Theorem 2.4.1.

To show the sharpness of Theorem 2.2.2, we let $H$ be the graph obtained from the Petersen graph $P$ by adding exactly one pendant edge to every vertex of $P$. Then $L(H)$ 
is a 3 -connected $\left\{K_{1,3}, Z_{9}\right\}$-free graph. However, $L(H)$ is non-hamiltonian.

\subsection{Concluding remark}

In the last paragraph of Section 2.4 we presented an extremal graph to show the sharpness of Theorem 2.4.1. However it has only 20 vertices. We believe it is the unique nonhamiltonian 3-connected $\left\{K_{1,3}, Z_{9}\right\}$-free graph. Hence we propose the following conjecture which implies Theorem 2.2.3 since a $P_{11}$-free graph must be $Z_{9}$-free and since the line graph $L(H)$ of a graph $H$ defined in Conjecture 2.5.1 is $P_{11}$-free.

Conjecture 2.5.1 If $G$ is 3-connected and $\left\{K_{1,3}, Z_{9}\right\}$-free, then $G$ is hamiltonian unless $G$ is the line graph of $H$ defined in the last paragraph of Section 2.4.

To show the sharpness of Conjecture 2.5.1, we let $F$ be a graph by adding some pendant edges to every vertex of the Petersen graph where at least one vertex of the Petersen graph is incident to at least 2 pendant edges. Then $\kappa(L(F)) \geq 3$ and $L(F)$ is claw-free and $Z_{10}$-free. Moreover, $L(F)$ has a $Z_{9}$ and $L(F)$ is non-hamiltonian.

Although we can not prove Conjecture 2.5.1 at this moment, we will prove Theorem 2.2.3 in a similar way to the proof of Theorem 2.2.2. Note that $L(G)$ is $P_{11}$-free if and only if $G$ has no subgraph isomorphic to $P_{12}$. Hence in order to prove Theorem 2.2.3, it suffices to prove the following result by Theorems 2.1.1 and 2.1.2.

Theorem 2.5.2 Let $G$ be a connected simple graph without subgraphs isomorphic to $P_{12}$. If $\kappa(L(G)) \geq 3$, then $L(G)$ is hamiltonian.

Proof of Theorem 2.5.2. Let $G_{0}$ be the core of $G$. To show $L(G)$ is hamiltonian, by Theorems 1.1.3 and 1.1.2, it suffices to show the following claim.

Claim 4. $G_{0}$ has a dominating closed trail $H$ such that $V(H)$ contains all nontrivial vertices and end-vertices of all nontrivial edges of $G_{0}$. 
We argue by contradiction, and assume that $G_{0}$ is a counter-example to Claim 4 with $\left|V\left(G_{0}\right)\right|$ minimized.

From the proof of Theorem 2.4.1, we can suppose that $G_{0}$ is reduced, $\kappa^{\prime}\left(G_{0}\right) \geq 3$ and $c\left(G_{0}\right) \geq 9$. It suffices to consider the case that $c\left(G_{0}\right) \geq 10$ by Lemma 2.4.3. Note that $G_{0}$ has no spanning closed trails by its choice.

If $c\left(G_{0}\right) \geq 11$, then $G_{0}$ has a path $P_{12}$ since $G_{0}$ has at least one vertex not on the longest cycle.

So we can suppose that $c\left(G_{0}\right)=10$. Then every cycle of length 10 is dominating since otherwise $G_{0}$ has a $P_{12}$ obtained by the longest cycle and a path containing an un-dominated edge. Hence there is a vertex $w$ which is either a nontrivial vertex or one end-vertex of a nontrivial edge of $G_{0}$. If $w$ is a nontrivial vertex, then $w$ has at least a neighbor $w^{\prime}$ in the original graph $G$; if $w$ is one end-vertex of a nontrivial edge of $G_{0}$, then there is a path of length 2 whose internal vertex $w^{\prime}$ has degree two in the original graph $G$. In either case we obtain a path $P_{12}$ in the original graph $G$, a contradiction. This completes the proof of Claim 4 and (hence) of Theorem 2.5.2, which implies that every 3 -connected $\left\{K_{1,3}, P_{11}\right\}$-free graph is hamiltonian. Hence Theorem 2.2.3 is proved.

The graph $F$ also shows the sharpness of Theorem 2.5.2. 


\section{Chapter 3}

\section{Hamilton-connected line graphs}

\subsection{Introduction}

A lane in $G$ is a nontrivial trail whose ends are not in $D_{2}(G)$ and whose internal vertices, if any, have degree 2 in $G$ (and thus are in $D_{2}(G)$ ). Note that a lane may be a cycle. If the lane has length 1 , then it has no internal vertices. The length of a lane is defined to be the number of its edges.

Corollary 3.1.1 Let $G$ be a connected graph that is not a cycle. For any $m \geq 0$ if $L^{m}(G)$ is Hamilton-connected, then $L^{n}(G)$ is Hamilton-connected for all $n \geq m$.

We say that an edge $e \in E(G)$ is subdivided when it is replaced by a path of length 2 whose internal vertex, denote $v(e)$, has degree 2 in the resulting graph. The resulting new two edges are denoted by $e^{\prime}$ and $e^{\prime \prime}$. The process of taking an edge $e$ and replacing it by the path of length 2 is called subdividing $e$. For a graph $G$ and edges $e_{1}, e_{2} \in E(G)$, let $G\left(e_{1}\right)$ denote the graph obtained from $G$ by subdividing $e_{1}$, and let $G\left(e_{1}, e_{2}\right)$ denote the graph obtained from $G$ by subdividing both $e_{1}$ and $e_{2}$. Thus

$$
V\left(G\left(e_{1}, e_{2}\right)\right)-V(G)=\left\{v\left(e_{1}\right), v\left(e_{2}\right)\right\} .
$$

From the definitions, one immediately has the following observation. 
Proposition 3.1.2 For a graph $G$ and two edges $e_{1}, e_{2} \in E(G)$, if $G\left(e_{1}, e_{2}\right)$ has a spanning $\left(v\left(e_{1}\right), v\left(e_{2}\right)\right)$-trail, then $G$ has a spanning $\left(e_{1}, e_{2}\right)$-trail.

In 1983, Clark and Wormald [22] introduced the concept of Hamilton-connected index. Let $G$ be an undirected graph that is neither a path nor a cycle. The hamiltonian index $h(G)$ (Hamilton-connected index $h c(G)$, respectively) is the least nonnegative integer $k$ such that $L^{k}(G)$ is hamiltonian (Hamilton-connected, respectively).

Theorem 3.1.3 (Combining Catlin, Janakiraman and Srinivasan, [13], and Lai, [34]) Let $G$ be a connected graph that is neither a path nor $C_{2}$. Let $k$ be the length of the longest lane in $G$. Then $h(G) \leq \min \{\operatorname{diam}(G), k+1\}$.

In this chapter, we consider the Hamilton-connected index of a graph. In Section 3.2, we get some results of Hamilton-connected index associated with diameter. In Section 3.3, we present the relationship between the Hamilton-connected index and the connectivity of a graph. In Section 3.4, we give some relations between the Hamilton-connected index and the minimum and maximum degrees of a graph.

Lemma 3.1.4 If $G$ is collapsible, then for any pair of vertices $u, v \in V(G), G$ has a spanning $(u, v)$-trail.

Proof. See (iii) of Theorem 1.2.1.

Lemma 3.1.5 Let $H$ be a collapsible subgraph of a graph $G$ and $H^{\prime}=G / H$. Let $u, v \in$ $V(G)$ and $u^{\prime}, v^{\prime} \in V\left(H^{\prime}\right)$ such that $u^{\prime}, v^{\prime}$ are the images of $u, v$ respectively. Then $G$ has a spanning $(u, v)$-trail if and only if $H^{\prime}$ has a spanning $\left(u^{\prime}, v^{\prime}\right)$-trail.

Proof. It is clear that $H^{\prime}$ has a spanning $\left(u^{\prime}, v^{\prime}\right)$-trail if $G$ has a spanning $(u, v)$-trail. So we only need to prove that $G$ has a spanning $(u, v)$-trail if $H^{\prime}$ has a spanning $\left(u^{\prime}, v^{\prime}\right)$-trail.

Suppose that $\Gamma^{\prime}$ is a spanning $\left(u^{\prime}, v^{\prime}\right)$-trail in $H^{\prime}$. Take one vertex $w_{0} \notin V(G)$ and let $\Gamma^{\prime \prime}$ be a trail in $H^{\prime}$ with $V\left(\Gamma^{\prime \prime}\right)=V\left(\Gamma^{\prime}\right) \cup\left\{w_{0}\right\}$ and $E\left(\Gamma^{\prime \prime}\right)=E\left(\Gamma^{\prime}\right) \cup\left\{u^{\prime} w_{0}, v^{\prime} w_{0}\right\}$ 
if $u^{\prime} \neq v^{\prime}$, and let $\Gamma=\left\{\begin{array}{ll}\Gamma^{\prime}, & \text { if } u^{\prime}=v^{\prime} \\ \Gamma^{\prime \prime}, & \text { if } u^{\prime} \neq v^{\prime}\end{array}\right.$. Then $\Gamma$ is eulerian. Let $S=\{w \in V(H)$ : $w$ is incident with an odd number of edges in $E(\Gamma)\}$. Then $|S|$ is even and $S \triangle O(H)$ is even too. Note that $H$ is collapsible. Then there exists $L \subseteq H$ such that $L$ is a connected, spanning subgraph in $H$ such that $O(L)=S \triangle O(H)$. Thus $\Gamma \cup L$ is a spanning eulerian subgraph in $G+w_{0}$. Therefore $G$ has a spanning $(u, v)$-trail.

\subsection{Hamilton-connected index and diameter}

Let $G$ be a graph. Denote $E^{\prime}=E^{\prime}(G)=\{e \in E(G): e$ is in a cycle of $G$ of length at most 3$\}$ and $E^{\prime \prime}=E(G)-E^{\prime}(G)$.

Let $H$ be an induced subgraph of $G$. The subgraph induced by the vertex set $E(H)$ in $L(G)$, denoted by $I_{1}(H)$, is called the 1 -line-image of $H$, and $H$, denoted by $I_{1}^{-1}\left(I_{1}(H)\right)$, is called the 1-line-pre-image of $I_{1}(H)$. The subgraph induced by the vertex set $E\left(I_{1}(H)\right)$ in $L^{2}(G)$, denoted by $I_{2}(H)$, is called the 2-line-image of $H$, and $H$, denoted by $I_{2}^{-1}\left(I_{2}(H)\right)$, is called the 2-line-pre-image of $I_{2}(H)$. Generally, the subgraph induced by the vertex set $E\left(I_{k}(H)\right)$ in $L^{k+1}(G)$, denoted by $I_{k+1}(H)$, is called the $(k+1)$-line-image of $H$. Conversely, $H$, denoted by $I_{k+1}^{-1}\left(I_{k+1}(H)\right)$, is called the $(k+1)$-line-pre-image of $I_{k+1}(H)$. We adopt $I_{k+1}^{-1}(e)$ when $I_{k+1}(H)$ is a path induced by an edge $e$.

Lemma 3.2.1 Let $P$ be a lane in $G$ with length $d$. Then $I_{k}(P)(k \leq d)$ is a lane in $L^{k}(G)$ with length $(d-k)$. Particularly, $I_{d-1}(P) \in E^{\prime \prime}\left(L^{d-1}(G)\right)$.

Lemma 3.2.2 Let $e \in E^{\prime \prime}\left(I^{d-1}(G)\right)$. Then $I_{d-1}^{-1}(e)$ is a lane in $G$ with length $d$.

Theorem 3.2.3 Let $G$ be a connected graph that is neither a path nor $C_{n}$. If the length of a longest lane is $k$, then $k-1 \leq h c(G) \leq \max \{\operatorname{diam}(G), k-1\}$.

Proof. Since a longest lane of length $k$ in $G$ becomes a lane of length 2 in $L^{k-2}(G)$, and so $L^{k-2}(G)$ is not Hamilton-connected, $h c(G) \geq k-1$. 
The proof of the second inequality remains. If $\operatorname{diam}(G)=1$, then $G$ is spanned by $K_{n}$. Thus $h c(G)=0 \leq \max \{\operatorname{diam}(G), k-1\}$. Next we prove that the theorem holds for $d=\max \{\operatorname{diam}(G), k-1\} \geq 2$ by contradiction.

Let $f_{1}=u_{1} v_{1}, f_{2}=u_{2} v_{2} \in E\left(L^{d-1}(G)\right)$ and $H$ be the reduction of $L^{d-1}(G)\left(f_{1}, f_{2}\right)$. By Lemma 3.1.4, Proposition 3.1.2 and Theorem 1.1.4, $H \neq K_{1}$. Note that

$$
d_{L^{d-1}(G)\left(f_{1}, f_{2}\right)}\left(v\left(f_{i}\right)\right)=2(i=1,2) .
$$

Then either $\left\{f_{i}^{\prime}, f_{i}^{\prime \prime}\right\} \cap E(H)=\emptyset$ or $\left\{f_{i}^{\prime}, f_{i}^{\prime \prime}\right\} \subseteq E(H)(i=1,2)$. Let $H^{\prime}=H /(E(H) \cap$ $\left.\left\{f_{1}^{\prime \prime}, f_{2}^{\prime \prime}\right\}\right)$. Then $H^{\prime} \neq K_{2}$.

Claim 1. $E\left(H^{\prime}\right) \subseteq E\left(L^{d-1}(G)\right)$.

Proof. If $\left\{f_{1}^{\prime}, f_{1}^{\prime \prime}, f_{2}^{\prime}, f_{2}^{\prime \prime}\right\} \cap E(H)=\emptyset$, then $H^{\prime}=H$. Thus $E\left(H^{\prime}\right) \subseteq E\left(L^{d-1}(G)\right)$. If $\left\{f_{1}^{\prime}, f_{1}^{\prime \prime}, f_{2}^{\prime}, f_{2}^{\prime \prime}\right\} \cap E(H) \neq \emptyset$, without loss of generality, we assume $\left\{f_{1}^{\prime}, f_{1}^{\prime \prime}\right\} \subseteq E(H)$. Note that $H^{\prime}=H /\left(E(H) \cap\left\{f_{1}^{\prime \prime}, f_{2}^{\prime \prime}\right\}\right)$. Then $f_{1}=f_{1}^{\prime} \in E\left(H^{\prime}\right)$. Thus Claim 1 holds.

By the definition of $E^{\prime}\left(H^{\prime}\right)$ and Theorem 1.2.2(ii), we have the following claim.

Claim 2. Let $e \in E^{\prime}\left(H^{\prime}\right)$. If $e$ is in some 3-cycle of $H^{\prime}$, then this cycle contains $f_{1}^{\prime}$ or $f_{2}^{\prime}$. If $e$ is in a 2-cycle, then the two edges of the 2-cycle are $f_{1}^{\prime}$ and $f_{2}^{\prime}$.

Claim 3. $E\left(H^{\prime}\right)-E^{\prime}\left(L^{d-1}(G)\right) \neq \emptyset$.

Proof. By contradiction. Suppose that $E\left(H^{\prime}\right) \subseteq E^{\prime}\left(L^{d-1}(G)\right)$. Let $e \in E\left(H^{\prime}\right)$. As $E^{\prime \prime}\left(H^{\prime}\right) \subseteq E^{\prime \prime}(H) \subseteq E^{\prime \prime}\left(L^{d-1}(G)\right)$, we have $e \in E^{\prime}\left(H^{\prime}\right)$. We consider three cases.

Case 1. $e$ is in a 2-cycle of $H^{\prime}$.

Note that an $m$-cycle with $m \leq 3$ is collapsible. By Claim 2 and the assumption that $E\left(H^{\prime}\right) \subseteq E^{\prime}\left(L^{d-1}(G)\right), H^{\prime}$ must be the graph shown in Figure 1. Thus, there exists a spanning $\left(v\left(f_{1}\right), v\left(f_{2}\right)\right)$-trail in $L^{d-1}(G)\left(f_{1}, f_{2}\right)$ by Lemma 3.1.5, a contradiction. 


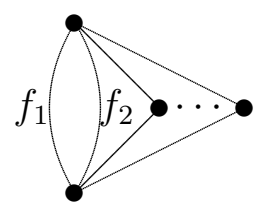

Figure 1

Case 2. $e$ is in a 3-cycle of $H^{\prime}$ containing exactly one of $f_{1}^{\prime}$ and $f_{2}^{\prime}$.

Without loss of generality, we assume that this cycle contains $f_{1}^{\prime}$ only. Note again that an $m$-cycle with $m \leq 3$ is collapsible. By the assumption that $E\left(H^{\prime}\right) \subseteq E^{\prime}\left(L^{d-1}(G)\right)$, the graph $H^{\prime}$ must be the graph shown in Figure 2, where $v\left(f_{2}\right)$ is in the pre-image of some vertex. Thus, there exists a spanning $\left(v\left(f_{1}\right), v\left(f_{2}\right)\right)$-trail in $L^{d-1}(G)\left(f_{1}, f_{2}\right)$ by Lemma 3.1.5, a contradiction.

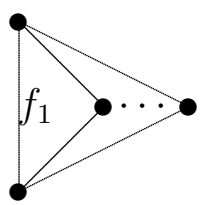

Figure 2

Case 3. $e$ is in a 3-cycle of $H^{\prime}$ containing both $f_{1}^{\prime}$ and $f_{2}^{\prime}$.

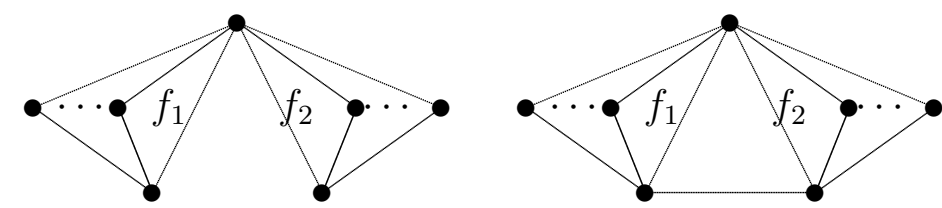

Figure 3

Suppose that $f_{1}$ and $f_{2}$ are incident. Then the graph $H^{\prime}$ must be one of the graphs in Figure 3. Thus, there exists a spanning $\left(v\left(f_{1}\right), v\left(f_{2}\right)\right)$-trail in $L^{d-1}(G)\left(f_{1}, f_{2}\right)$ by Lemma 3.1.5, a contradiction. Now suppose that $f_{1}$ and $f_{2}$ are not incident. Then the graph $H^{\prime}$ must be one of the graphs in Figure 4. Again, there exists a spanning $\left(v\left(f_{1}\right), v\left(f_{2}\right)\right)$-trail in $L^{d-1}(G)\left(f_{1}, f_{2}\right)$ by Lemma 3.1.5, a contradiction. So Claim 3 holds.
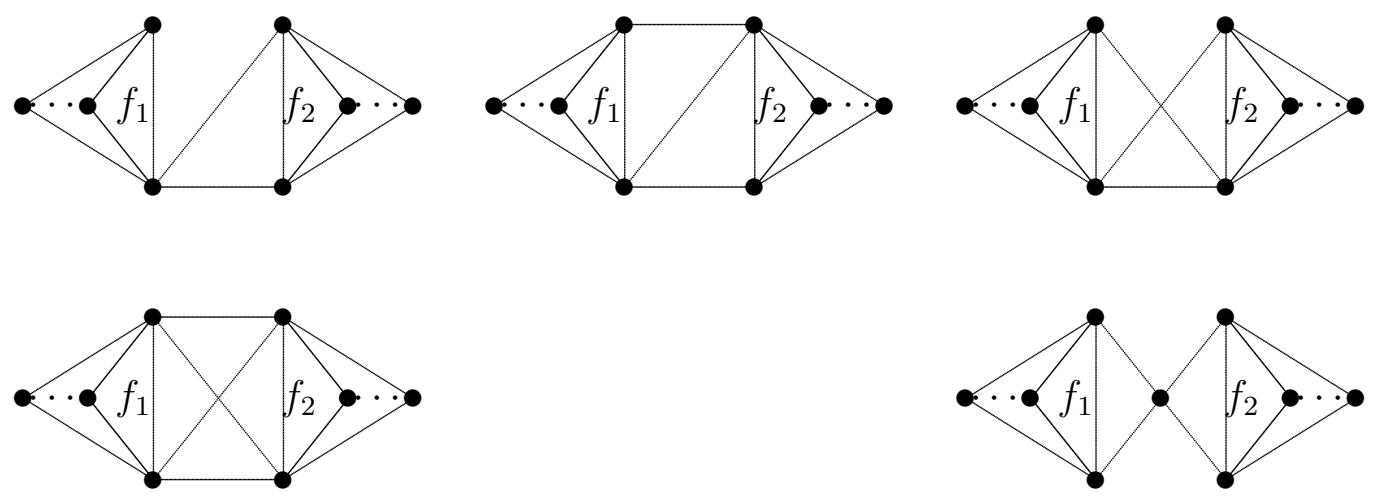

Figure 4 
By Claim 3, let $e \in E\left(H^{\prime}\right)-E^{\prime}\left(L^{d-1}(G)\right)$. Then $I_{d-1}^{-1}(e)$ is a lane in $G$ with length at least $d$. Let $P_{1}=(u, v)$-path be the maximal lane in $G$ containing $I_{d-1}^{-1}(e)$. Suppose first $P_{1}$ is $(u, v)$-path (for the case that $P_{1}$ is a cycle, we can argue similarly). Then $d_{G}(u), d_{G}(v) \geq 3$, and at least there exists another $(v, u)$-path $P_{2}$ in $G-E\left(P_{1}\right)$ (otherwise, let $u_{1} \in N_{G}(u)-V\left(P_{1}\right)$ and $v_{1} \in N_{G}(v)-V\left(P_{1}\right)$, then $\operatorname{dist}\left(u_{1}, v_{1}\right)=d+2$, a contradiction). Let $P_{2}, \cdots, P_{m}$ be all minimal $(u, v)$-paths in $G-E\left(P_{1}\right)$ with $E\left(P_{i}\right) \cap E\left(P_{j}\right)=\emptyset$ (when $i \neq j)$.

Claim 4. There exists a $(u, v)$-path $P_{i}(i \neq 1)$ that contains a lane with length at most $d-1$.

Proof. By contradiction. Suppose that each $P_{i}$ contains a lane with length at least d. Then $1 \leq\left|L^{d-1}\left(P_{i}\right) \cap E^{\prime \prime}\left(L^{d-1}(G)\right)\right| \leq 2$ and $\sum_{i=1}^{m}\left|L^{d-1}\left(P_{i}\right) \cap E^{\prime \prime}\left(L^{d-1}(G)\right)\right| \leq m+1$ since $d \geq \operatorname{diam}(G)$. If $m=2$, noting that $d_{G}(u) \geq 3, d_{G}(v) \geq 3$, so there always exist some $x \in N_{G}(u)$ and $y \in N_{G}(v)$ such that $\operatorname{dist}_{G}(x, y) \geq d+1>\operatorname{diam}(G)$, a contradiction. So $m \geq 3$. If $f_{i} \notin \bigcup_{i=1}^{m} E\left(L^{d-1}\left(P_{i}\right)\right)$, then $e \notin E\left(H^{\prime}\right)$ since $m \geq 3$. So $\left\{f_{1}, f_{2}\right\} \subseteq \bigcup_{i=1}^{m} E\left(L^{d-1}\left(P_{i}\right)\right)$, and $H=C_{4}$ or $K_{2,3}$. If $H=K_{2,3}$, then there is a spanning $\left(v\left(f_{1}\right), v\left(f_{2}\right)\right)$-trail in $H$. If $H=C_{4}$, then one of vertices in $H$ is trivial. Thus there is a dominating $\left(v\left(f_{1}\right), v\left(f_{2}\right)\right)$-trail in $H$. In either case, $L^{d}(G)$ is Hamilton-connected, a contradiction. So Claim 4 holds.

By Claim 4, suppose that $P_{2}$ contains a lane with length at most $d-1$. Note that $P_{1} \cup P_{2}$ is a cycle in $G$. Then $L^{d-1}\left(P_{1} \cup P_{2}\right)$ is a still cycle in $L^{d-1}(G), e \in L^{d-1}\left(P_{1} \cup P_{2}\right)$ and at most two edges in $L^{d-1}\left(P_{1} \cup P_{2}\right)$ are not in $E^{\prime}\left(L^{d-1}(G)\right)$. If $m \geq 3$, then $H=C_{4}$, $v\left(f_{1}\right), v\left(f_{2}\right) \in V\left(C_{4}\right)$ and one of the vertices of $V(H)$ is trivial. Thus $L^{d}(G)$ is Hamiltonconnected. So we assume that $m=2$.

Claim 5. $\left|E\left(L^{d-1}\left(P_{1}\right)\right)\right|=2$.

Proof. By contradiction. Suppose that $E\left(L^{d-1}\left(P_{1}\right)\right)=\{e\}$. If $e \in\left\{f_{1}, f_{2}\right\}$, without loss of generality, we assume that $e=f_{1}$. Then $H$ is the graph $K_{2,3}$ (see Figure 5). Thus $L^{d-1}(G)$ has a spanning $\left(v\left(f_{1}\right), v\left(f_{2}\right)\right)$-trail by Lemma 3.1.5. Hence $L^{d}(G)$ is Hamiltonconnected, a contradiction. Thus $e \notin\left\{f_{1}, f_{2}\right\}$. 


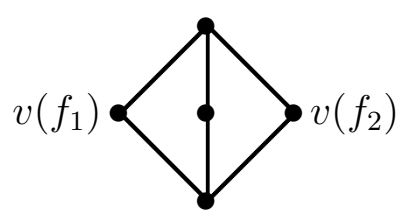

Figure 5

Since each edge except $e$ in $L^{d-1}\left(P_{1} \cup P_{2}\right)$ is in $E^{\prime}\left(L^{d-1}(G)\right)$ and $e \notin E^{\prime}\left(L^{d-1}(G)\right)$, we have $f_{1}, f_{2} \in E\left(L^{d-1}\left(P_{1} \cup P_{2}\right)\right)$. If $f_{1}, f_{2}$ are in the same triangle, then $L^{d-1}\left(P_{1} \cup P_{2}\right)$ is collapsible, thus $e \notin E(H)$, a contradiction. Thus $f_{1}, f_{2}$ are not in the same triangle. Hence $H$ is the graph shown in Figure 6 . Thus $L^{d-1}(G)$ has a spanning $\left(v\left(f_{1}\right), v\left(f_{2}\right)\right)$-trail by Lemma 3.1.5. Hence $L^{d}(G)$ is Hamilton-connected, a contradiction. So Claim 5 holds.

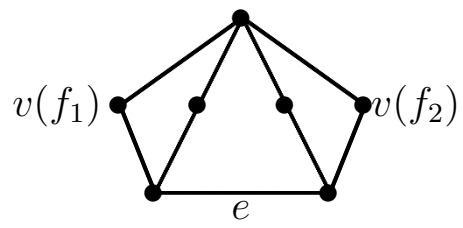

Figure 6

By Claim 5, $\left|E\left(L^{d-1}\left(P_{1}\right)\right)\right|=2$. Since each edge in $L^{d-1}\left(P_{1} \cup P_{2}\right)$ except $L^{d-1}\left(P_{1}\right)$ is in $E^{\prime}\left(L^{d-1}(G)\right)$ and $L^{d-1}\left(P_{1}\right) \cap E^{\prime}\left(L^{d-1}(G)\right)=\emptyset$, we have $\left|\left\{f_{1}, f_{2}\right\} \cap E\left(L^{d-1}\left(P_{1} \cup P_{2}\right)\right)\right| \geq 1$. If $\left|\left\{f_{1}, f_{2}\right\} \cap E\left(L^{d-1}\left(P_{1} \cup P_{2}\right)\right)\right|=1$, without loss of generality, we assume that $f_{1} \in$ $E\left(L^{d-1}\left(P_{1} \cup P_{2}\right)\right)$. Then $v\left(f_{2}\right)$ is contracted. Thus $f_{1} \notin E\left(L^{d-1}\left(P_{1}\right)\right)$. (Otherwise $H$ is collapsible and $H=K_{1}$.) Moreover $H=K_{2,3}$ and $x$ is trivial in $H$ (see Figure 7 ). Thus the pre-image of a vertex in $V(H)-\left\{x, v\left(f_{1}\right)\right\}$ contains $v\left(f_{2}\right)$. It is easy to check that $L^{d-1}(G)$ contains a dominating $\left(v\left(f_{1}\right), v\left(f_{2}\right)\right)$-trail by Lemma 3.1.5. Hence $L^{d}(G)$ is Hamiltonconnected. This contradicts the assumption. Thus $\left|\left\{f_{1}, f_{2}\right\} \cap E\left(L^{d-1}\left(P_{1} \cup P_{2}\right)\right)\right|=2$. 


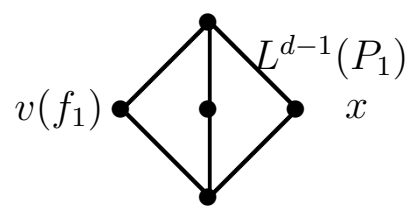

Figure 7

We break into three cases to finish the proof.

Case 1. $\left|\left\{f_{1}, f_{2}\right\} \cap E\left(L^{d-1}\left(P_{1}\right)\right)\right|=0$.

If $f_{1}, f_{2}$ are in the same triangle in $H^{\prime}$, then $H$ is the graph shown in Figure 8.

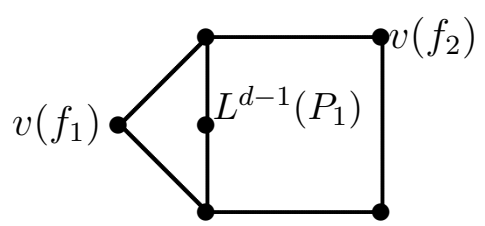

Figure 8

If $f_{1}, f_{2}$ are in two edge-disjoint triangles in $H^{\prime}$, then $H$ is one of the graphs in Figure 9. (In this and following figures, the two $\mathbf{s}$ stand for the vertices $v\left(f_{1}\right)$ and $v\left(f_{2}\right)$, respectively).
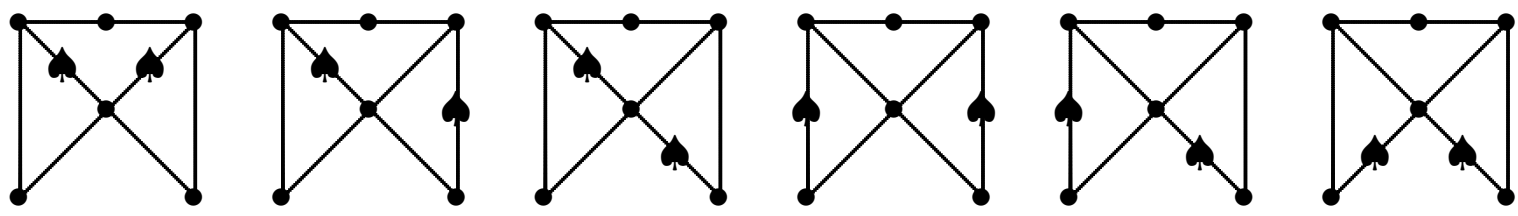

Figure 9

If $f_{1}, f_{2}$ are in two triangles sharing an edge in $H^{\prime}$, then $H$ is one of the graphs shown in Figure 10. 

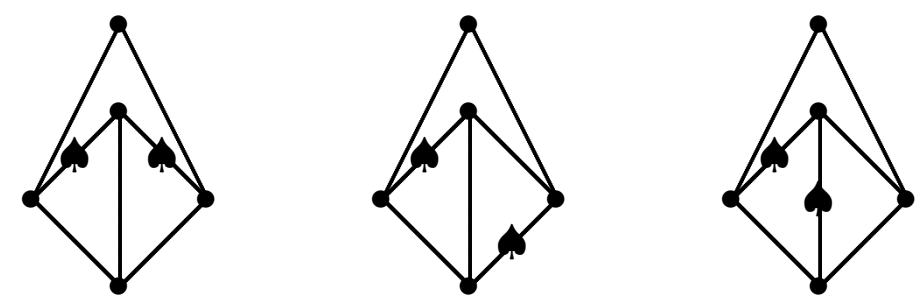

Figure 10

In either graph, $H$ contains a spanning $\left(v\left(f_{1}\right), v\left(f_{2}\right)\right)$-trail. Thus $L^{d}(G)$ is Hamiltonconnected, a contradiction.

Case 2. $\left|\left\{f_{1}, f_{2}\right\} \cap E\left(L^{d-1}\left(P_{1}\right)\right)\right|=1$.

In this case, $H$ is one of the graphs shown in Figure 11. Note that $L^{d-1}\left(P_{1}\right)$ is a lane. Then the vertex $x$ (see the graph) is trivial in $H$. Thus $L^{d-1}(G)$ contains a spanning $\left(v\left(f_{1}\right), v\left(f_{2}\right)\right.$-trail by Lemma 3.1.5. Hence $L^{d}(G)$ is Hamilton-connected, a contradiction.
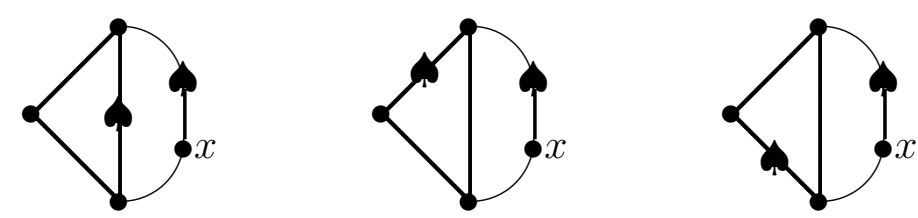

Figure 11

Case 3. $\left|\left\{f_{1}, f_{2}\right\} \cap E\left(L^{d-1}\left(P_{1}\right)\right)\right|=2$.

Then $H$ is the graph shown in Figure 12, and $x$ is trivial in $H$. Thus $L^{d}(G)$ is Hamilton-connected, a contradiction.

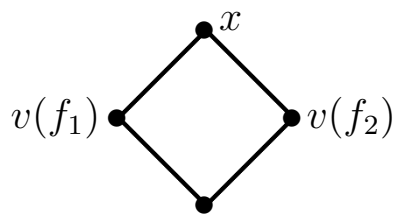

Figure 12

Having exhausted the cases, we have completed the proof of Theorem 3.2.3. 
An obvious corollary is the following.

Corollary 3.2.4 Let $G$ be a connected graph that is neither a path nor $C_{n}$. If the length of a longest lane is $k$ with $k \geq \operatorname{diam}(G)+1$, then $h c(G)=k-1$.

Noting that $k \leq 2 \operatorname{diam}(G)-1$, we have the following corollary.

Corollary 3.2.5 Let $G$ be a connected graph that is neither a path nor $C_{n}$. Then $h c(G) \leq$ $2(\operatorname{diam}(G)-1)$.

Let $C$ be a cycle of length $2 d(d>1)$ and $K$ be a complete graph of order $m>2$. $G$ is a graph obtained by combining $C$ and $K$ so that $C$ and $K$ share exactly one edge. Then $L^{2 d-3}(G)$ has a 2-cut, so $L^{2 d-3}(G)$ is not Hamilton-connected. On the other hand, $L^{2 d-2}(G)$ is Hamilton-connected. Therefore Corollary 3.2.5 is best possible.

Theorem 3.2.6 Let $d=\operatorname{diam}(G) \geq 3$. Then one of the following holds.

(i) $L^{d}(G)$ is Hamilton-connected;

(ii) $L^{d-1}(G)$ has a collapsible subgraph $H$ such that $L^{d-1}(G) / H$ is a cycle of length at least 3 .

Proof. Suppose (i) does not hold. It suffices to show (ii) holds. Since $L^{d}(G)$ is not Hamilton-connected, there exists a lane $L$ in $G$ with length $k \geq d+2$ by Theorem 3.2.3. Suppose $u$ and $v$ are the two end-vertices of $L$ (possibly $u=v$ if $L$ is a cycle), then $d(u) \geq 3$ and $d(v) \geq 3$ (otherwise $d>k$ ). Moreover, $G-L$ is connected. $L^{d-1}(L)$ is still a lane with length at least 3 and we assume $L^{\prime}=L^{d-1}(L)$ with two end-vertices $u^{\prime}$ and $v^{\prime}$ in $L^{d-1}(G)$. Let $H=\left(L^{d-1}(G)-L^{\prime}\right) \cup\left\{u^{\prime}, v^{\prime}\right\}$. We are going to show that $H$ is collapsible. Let $H^{\prime}$ be the reduction of $H$ and we only need to show $H^{\prime}=K_{1}$. For a contradiction, suppose there exists at least one edge $x y$ in $H^{\prime}$. Since $H^{\prime}$ is reduced, $x y$ can not be in a cycle of length at most 3. Correspondingly, there exists at least one edge $x^{\prime} y^{\prime}$ in the pre-image of $x y$ in $H$ that is not contained in a cycle of length at most 
3. By Lemma 3.2.2, the pre-image of $x^{\prime} y^{\prime}$ in $G$ must be a lane with length at least $d$ and suppose the lane is $Q$. Take the midpoint $w$ of $L$ and the midpoint $z$ of $Q$, then $\operatorname{dist}(w, z) \geq k / 2+d / 2 \geq d+1$, a contradiction. Note that $L^{d-1}(G) / H$ is a cycle obtained by identifying the two end-vertices of $L^{\prime}$ and has length at least three. So we are done.

Then we have the following corollary by the above theorem:

Corollary 3.2.7 Let $d=\operatorname{diam}(G) \geq 3$. Then $L^{d}(G)$ is Hamilton-connected if and only if $\kappa\left(L^{d}(G)\right) \geq 3$.

Proof. Necessity. This direction is trivial.

Sufficiency. For a contradiction, suppose that $\kappa\left(L^{d}(G)\right) \geq 3$ and $L^{d}(G)$ is not Hamilton-connected, then $L^{d-1}(G)$ is essentially 3-edge-connected. By (ii) of Theorem 3.2.6, $L^{d-1}(G)$ has an essential 2-edge-cut, a contradiction.

\subsection{Hamilton-connected index and connectivity}

Let $\kappa^{3}(G)=\min \left\{m \mid L^{m}(G)\right.$ is 3-connected $\}$. The following result shows that the Hamiltonconnected index of $G$ is not far from $\kappa^{3}(G)$.

Theorem 3.3.1 Let $G$ be a graph which is neither a path nor a cycle. Then $\kappa^{3}(G) \leq$ $h c(G) \leq \kappa^{3}(G)+2$.

Proof. Let $c=\kappa^{3}(G)$. Noticing that a Hamilton-connected graph should be 3-connected, we have $h c(G) \geq c$.

It suffices to prove that $h c(G) \leq c+2$. According to the definition of $c$, we know that $L^{c}(G)$ is 3 -connected. So $\delta\left(L^{c}(G)\right) \geq 3$. Then $L^{c+1}(G)$ is the union of edge-disjoint complete subgraphs and $\delta\left(L^{c+1}(G)\right) \geq 4, \kappa\left(L^{c+1}(G)\right) \geq 3$. Hence each edge of $L^{c+1}(G)$ is in a triangle and $L^{c+1}(G)$ is collapsible by Theorem 1.2.3. Let $H=L^{c+1}(G)$. For any two edges $e$ and $f$ in $H$, we distinguish the following two cases. 
Case 1. $e$ and $f$ are not in the same complete subgraph and, say, they are in two different complete subgraphs $K_{s}$ and $K_{t}$ of $H$ respectively.

If both $s$ and $t$ are at least 4 , then $H(e, f)$ is still collapsible since $K_{s}(e)$ and $K_{t}(f)$ have at least one triangle and exactly a $C_{4}$. Hence by Lemma 3.1.4, $H(e, f)$ has a spanning $(v(e), v(f))$-trail and so $L(H)=L^{c+2}(G)$ is Hamilton-connected. In the case that at least one of $\{s, t\}$ is 3 , say $s=3$, since $\kappa\left(L^{c+1}(G)\right) \geq 3$, the two end-vertices of $e$ are connected by a path $P$ with $E(P) \bigcap E\left(K_{s}\right)=\emptyset$ in which each edge is in a complete subgraph of order at least 3 , so the reduction of $H(e, f)$ is $K_{1}$, i.e., $H(e, f)$ is collapsible and so $L(H)=L^{c+2}(G)$ is Hamilton-connected.

Case 2. $e$ and $f$ are in the same complete subgraph $K_{t}$ of $H$.

If $t \geq 5$, then $H(e, f)$ is still collapsible since $K_{t}(e, f)$ has at least a triangle. Hence by Lemma 3.1.4 $H(e, f)$ has a spanning $(v(e), v(f))$-trail and so $L(H)=L^{c+2}(G)$ is Hamilton-connected. If $t=3$, since $\kappa\left(L^{c+1}(G)\right) \geq 3$, at least one of $\{e, f\}$, say $e$, has two end-vertices that are connected by a path $Q$ with $E(Q) \bigcap E\left(K_{t}\right)=\emptyset$ in which every edge is in a complete subgraph of order at least 3 , so the reduction of $H(e, f)$ is $K_{1}$, i.e., $H(e, f)$ is collapsible and hence $L(H)=L^{c+2}(G)$ is Hamilton-connected. In the remaining case that $t=4$, if $e$ and $f$ are incident, then the reduction of $H(e, f)$ is $K_{1}$ since $K_{t}(e, f)$ becomes a complete graph after contracting the unique triangle in it. Otherwise, if it is not collapsible, then the reduction of $H(e, f)$ must be the graph shown in Figure 13. Then it has a spanning $(v(e), v(f))$-trail, so $L(H)=L^{c+2}(G)$ is Hamilton-connected.

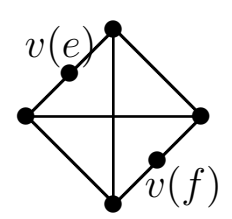

Figure 13

In either case, there is a spanning $(v(e), v(f))$-trail in $H(e, f)$. Hence $h c(G) \leq c+2$. 
To show the sharpness of Theorem 3.3.1, we present an infinite family of graphs $G$ with $h c(G)=\kappa^{3}(G)+2$. Let $P_{10}$ denote the Petersen graph and let $s \geq 1$ be an integer. Let $G(s)$ be obtained from $P_{10}$ by first replacing every edge of $P_{10}$ by a path of $s+1$ edges, and then adding a pendant edge at each vertex of $P_{10}$.

Then $l(G(s))=s+1$ and $k^{3}(G(s))=s$. However, $L^{s}(G(s))$ can be contracted to a $P_{10}$, each of whose vertices has a pre-image with at least 4 edges. Let $H_{1}$ be the pre-image of a vertex of $P_{10}$ viewed as a contraction image of $L^{s}(G(s))$ (in fact, $P_{10}$ is the reduction of $L^{s}(G(s))$ and we can just take $H_{1}$ as the pre-image of any vertex in the reduction). Take two edges $e_{1}, e_{2}$ of $L^{s}(G(s))$ such that $e_{1}, e_{2} \in V\left(H_{1}\right)$. Let $T$ be an $\left(e_{1}, e_{2}\right)$-trail of $L^{s}(G(s))$. In the process when $L^{s}(G(s))$ is contracted to $P_{10}, T$ is also contracted to an even subgraph $T^{\prime}$ (and so a cycle) of $P_{10}$, by the choices of $e_{1}$ and $e_{2}$. It follows that $T^{\prime}$ must miss at least one vertex of $P_{10}$, and so $T$ cannot be an dominating $\left(e_{1}, e_{2}\right)$ trail of $L^{s}(G(s))$. This proves that $L^{s+1}(G(s))$ cannot be hamiltonian connected, and so $h c(G(s))>\kappa^{3}(G(s))+1$. By Theorem 3.3.1, $h c(G(s))=\kappa^{3}(G(s))+2$.

\subsection{Hamilton-connected index and degree}

We start with some results on the hamiltonian index.

Theorem 3.4.1 (Chartrand and Wall, [15]) Let $G$ be a connected graph with minimum degree at least 3. Then $h(G) \leq 2$.

Theorem 3.4.2 (Saražin, [43]) Let $G$ be a connected graph that is not a path. Then $h(G) \leq|V(G)|-\Delta(G)$.

Accordingly, we have the following two theorems:

Theorem 3.4.3 Let $G$ be a connected graph with minimum degree at least 3. Then $h c(G) \leq 3$. 
Proof. Let $d, f \in E\left(L^{2}(G)\right)$ and $H$ be the reduction of $L^{2}(G)(e, f)$. Since $\delta(G) \geq 3$, $\delta(L(G)) \geq 4$. Thus every edge in $L^{2}(G)$ lies in some $K_{t}(t \geq 4)$. According to the proof of Theorem 3.3.1, $K_{t}(e)$ and $K_{t}(f)$ are collapsible. If $H$ is not collapsible, then it must be as Figure 13. Thus $L^{3}(G)$ is Hamilton-connected. This completes the proof of Theorem 3.4.3.

In the graph shown in Figure 14, each cycle stands for complete graphs $K_{t}(t \geq 4)$. Then $\delta(G) \geq 3$. On the other hand, $L^{2}(G)$ is not Hamilton-connected since $L^{2}(G)$ is not 3-connected, but $L^{3}(G)$ is Hamilton-connected. So Theorem 3.4.3 is best possible.

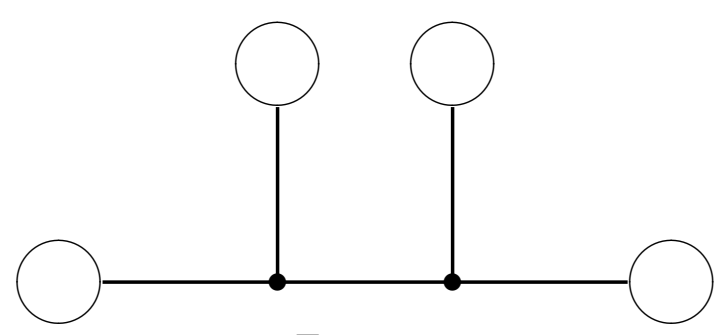

Figure 14

Theorem 3.4.4 Let $G$ be a connected graph that is neither a path nor a cycle. Then $h c(G) \leq|V(G)|-\Delta(G)+1$.

Proof. Since $G$ is a connected graph that is neither a path nor a cycle, $\Delta(G) \geq 3$. Let $u \in V(G)$ with $d_{G}(u)=\Delta(G)$, and $L$ be a longest lane with length $k$. Then

$$
\begin{gathered}
\left|\left(N_{G}(u) \cup\{u\}\right) \cap V(L)\right| \leq\left\{\begin{array}{ll}
2, & \text { if } L \text { is a path } \\
3, & \text { if } L \text { is a cycle }
\end{array},\right. \\
|V(L)|= \begin{cases}k+1, & \text { if } L \text { is a path } \\
k, & \text { if } L \text { is a cycle }\end{cases}
\end{gathered}
$$


and $\left|N_{G}(u) \cup\{u\}\right|=\Delta(G)+1$. Thus

$$
\begin{aligned}
\left|\left(N_{G}(u) \cup\{u\}\right) \cup V(L)\right| & =\left|N_{G}(u) \cup\{u\}\right|+|V(L)|-\left|\left(N_{G}(u) \cup\{u\}\right) \cap V(L)\right| \\
& \geq \begin{cases}(\Delta+1)+(k+1)-2, & \text { if } L \text { is a path } \\
(\Delta+1)+k-3, & \text { if } L \text { is a cycle }\end{cases} \\
& \geq \Delta+k-2 .
\end{aligned}
$$

Therefore $n \geq \Delta(G)+k-2$. If $\operatorname{diam}(G) \leq k-1$, then $h c(G) \leq k-1 \leq n-\Delta(G)+1$ by Theorem 3.2.3. Next we assume that $\operatorname{diam}(G) \geq k$. Then $h c(G) \leq \operatorname{diam}(G)$.

Let $Q$ be a $\left(v_{1}, v_{2}\right)$-path satisfying $d_{G}\left(v_{1}, v_{2}\right)=\operatorname{diam}(G)$. Let $\left|\left(N_{G}(u) \cup\{u\}\right) \cap V(Q)\right|=$ t. Then $\left|\left(N_{G}(u) \cup\{u\}\right) \cup V(Q)\right|=\left|N_{G}(u) \cup\{u\}\right|+|V(Q)|-\left|\left(N_{G}(u) \cup\{u\}\right) \cap V(Q)\right|=$ $(\Delta+1)+(\operatorname{diam}(G)+1)-t=\Delta+\operatorname{diam}(G)+2-t$. If $t \leq 2$, then $h c(G) \leq \operatorname{diam}(G) \leq$ $n-\Delta(G)-2+t \leq n-\Delta$. Thus we assume $t \geq 3$. Now we only need to discuss two cases.

Case 1. $u \notin V(Q)$

Then $\left|N_{G}(u) \cap V(Q)\right|=t$, so we can assume $\left\{u_{1}, u_{2}, \cdots, u_{t}\right\}=N_{G}(u) \cap V(Q)$ such that $u_{1}, u_{2}, \cdots, u_{t}$ occur on $V(Q)$ in the order of the indices. Note that $Q$ is a path satisfying $d_{G}\left(v_{1}, v_{2}\right)=\operatorname{diam}(G)$. By the choice of $Q$, we have $u_{i} u_{i+1} \in E(Q)(1 \leq i \leq t-1)$ and $t$ must be 3 . (Otherwise, if $t \geq 4$, then $d_{G}\left(v_{1}, v_{2}\right)$ can be shortened by discarding vertices $u_{2}, u_{3}, \cdots, u_{t-1}$ and adding $u$ to $Q$.) Thus $Q^{\prime}=Q-u_{2}+u$ is still a $\left(v_{1}, v_{2}\right)$ path satisfying $d_{G}\left(v_{1}, v_{2}\right)=\operatorname{diam}(G)$. Note that $n \geq\left|\left(N_{G}(u) \cup\{u\}\right) \cup V\left(Q^{\prime}\right)\right|$. Then $n \geq \Delta(G)+\operatorname{diam}(G)+2-t=\Delta(G)+\operatorname{diam}(G)-1$. Thus $h c(G) \leq n-\Delta(G)+1$.

Case 2. $u \in V(Q)$

Then $\left|N_{G}(u) \cap V(Q)\right|=t-1$, so we can assume $\left\{u_{1}, u_{2}, \cdots, u_{t-1}\right\}=N_{G}(u) \cap V(Q)$ such that $u_{1}, u_{2}, \cdots, u_{t-1}$ occur on $V(Q)$ in the order of the indices. Note that $Q$ is a path satisfying $d_{G}\left(v_{1}, v_{2}\right)=\operatorname{diam}(G)$ and $u$ is also on the path. It forces $t$ to be 3 and $u_{1} u, u u_{2} \in E(Q)$. Note that $n \geq\left|\left(N_{G}(u) \cup\{u\}\right) \cup V(Q)\right|$. Then $n \geq \Delta(G)+\operatorname{diam}(G)+$ $2-t=\Delta(G)+\operatorname{diam}(G)-1$. Thus $h c(G) \leq n-\Delta(G)+1$.

Let $G_{0}$ be the (unique) tree on $n \geq 5$ vertices with exactly 3 leaves such that there are two leaves of $G_{0}$ having distances $n-2$ from the third leaf. We have that $n=\left|V\left(G_{0}\right)\right|$, $\Delta(G)=3$ and $L^{n-\Delta\left(G_{0}\right)}\left(G_{0}\right)$ is not 3-connected (hence not Hamilton-connected) and 
$L^{n-\Delta\left(G_{0}\right)+1}\left(G_{0}\right)$ is Hamilton-connected. For the case that $n=5$, see Figure 15 . So Theorem 3.4.4 is best possible.

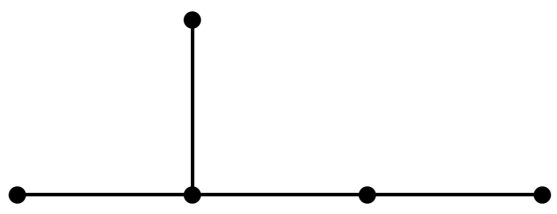

Figure 15 


\section{Chapter 4}

\section{The Chvátal-Erdös condition for supereulerian graphs}

\subsection{Introduction}

We only consider simple graphs $G$ with $\kappa(G) \geq 2$ in the whole chapter. Chvátal and Erdös gave the following well-known sufficient condition for a graph to be hamiltonian.

Theorem 4.1.1 (Chvátal and Erdös, [21]) If $\kappa(G) \geq \alpha(G)$, then $G$ is hamiltonian.

There are many extensions of Theorem 4.1.1, one of which aims at showing what properties a graph has when it satisfies a stronger Chvátal-Erdös condition.

Theorem 4.1.2 (Häggkvist and Thomassen, [28]) Let $t$ be a nonnegative integer. If $\kappa(G) \geq \alpha(G)+t$, then any system of disjoint paths of total length at most $t$ is contained in a hamiltonian cycle of $G$.

An immediate consequence of Theorem 4.1.2 is that $G$ is Hamilton-connected if $\kappa(G) \geq$ $\alpha(G)+1$. 
The first aim of this chapter is to consider whether a graph with a slightly weaker Chvátal-Erdös condition is supereulerian.

Theorem 4.1.3 If $\kappa(G) \geq \alpha(G)-1$, then exactly one of the following holds.

(a) $G$ is supereulerian;

(b) $G \in\left\{\right.$ the Petersen graph, $\left.K_{2,3}, K_{2,3}(1), K_{2,3}(2), K_{2,3}^{\prime}\right\}$, see Figures 4.1 and 4.2;

(c) $G$ is one of the two 2-connected graphs obtained from $K_{2,3}$ and $K_{2,3}(1)$ by replacing a vertex whose neighbors have degree three in $K_{2,3}$ and $K_{2,3}(1)$ with a complete graph of order at least three.

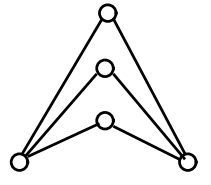

$K_{2,3}$

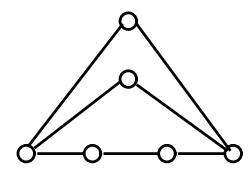

$\mathrm{K}_{2,3}(1)$

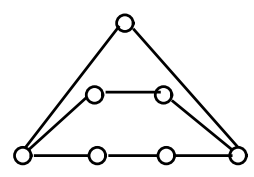

$\mathrm{K}_{2,3}(2)$

Figure 4.1: Three graphs $K_{2,3}, K_{2,3}(1), K_{2,3}(2)$

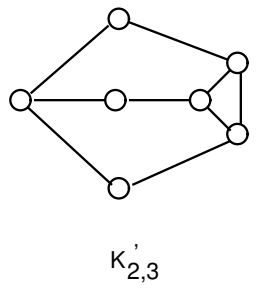

Figure 4.2: The graph $K_{2,3}^{\prime}$

The following known result is a consequence of Theorem 4.1.3.

Theorem 4.1.4 (Benhocine and Fouquet, [2]) If $\kappa(G) \geq \alpha(G)-1$, then $L(G)$ is hamiltonian, i.e., $h(G) \leq 1$. 
The following result extends Theorem 4.1.1 for triangle-free graphs, showing that a graph with a weaker Chvátal-Erdös condition has a weaker result.

Theorem 4.1.5 (Enomoto, Kaneko, Saito and Wei, [24]) If $G$ is a 2-connected trianglefree graph of order $n$ with $\kappa(G) \geq \frac{\alpha(G)+2}{2}$, then every longest cycle $C$ in $G$ is dominating (i.e., every edge of $G$ has at least one end vertex in $C$ ), and $G$ has a cycle of length at least $\min \{n-\alpha(G)+\kappa(G), n\}$.

There are many other ways to extend Theorem 4.1.1 (see the survey paper [31] and updated papers [1], [30], [33] and [46]). Our second aim in this chapter is to extend Theorems 4.1 .1 and 4.1 .4 by presenting an upper bound for $h(G)$.

Theorem 4.1.6 Let $t$ be a nonnegative integer. If $\kappa(G) \geq \alpha(G)-t$, then $h(G) \leq\left\lfloor\frac{2 t+2}{3}\right\rfloor$.

To show our main results, we need the following.

Theorem 4.1.7 (Jaeger, [32]) If $G$ is a 4-edge-connected graph, then $G$ is supereulerian.

Theorem 4.1.8 (Chen, [18]) If $G$ is a reduced graph and $\alpha(G) \geq 4$, then we have $(\delta(G) \alpha(G)+4) / 2 \leq|V(G)| \leq 4 \alpha(G)-5$.

Theorem 4.1.9 (Chen, [17]) Let $G$ be the graph of order $n \leq 11$. If $\kappa^{\prime}(G) \geq 3$, then $G$ is either collapsible or the Petersen graph.

The following theorem is obvious.

Theorem 4.1.10 Let $G^{\prime}$ be the reduction of $G$. Then, each of the following holds.

(a) $\alpha\left(G^{\prime}\right) \leq \alpha(G)$;

(b) If $G^{\prime}$ is nontrivial, then $\kappa^{\prime}\left(G^{\prime}\right) \geq \kappa^{\prime}(G)$. 
For any two subgraphs $H_{1}$ and $H_{2}$ of $G$, the distance $d_{G}\left(H_{1}, H_{2}\right)$ between $H_{1}$ and $H_{2}$ is defined to be the minimum of the distances $d_{G}\left(v_{1}, v_{2}\right)$ over all pairs with $v_{1} \in V\left(H_{1}\right)$ and $v_{2} \in V\left(H_{2}\right)$. If $d_{G}(e, H)=0$ for an edge $e$ of $G$, then we say that $H$ dominates $e$.

By Theorem 1.1.2, all supereulerian graphs and the Petersen graph have hamiltonian line graphs, and those graphs obtained from $K_{2,3}$ described in Theorem 4.1.3 have hamiltonian line graphs, which shows that Theorem 4.1.4 is a consequence of Theorem 4.1.3.

In this chapter, a branch is the same as a lane we defined previously. We denote by $\mathcal{B}(G)$ the set of branches of $G$ and by $\mathcal{B}_{1}(G)$ the subset of $\mathcal{B}(G)$ in which every branch has an end vertex in $V_{1}(G)$. For any subgraph $H$ of $G$, we denote by $\mathcal{B}_{H}(G)$ the set of branches of $G$ whose edges are all in $H$.

The following theorem can be considered as an analogue of Theorem 1.1.2 for $L^{k}(G)$.

Theorem 4.1.11 (Xiong and Liu, [47]) Let $G$ be a connected graph that is not a path, and let $k \geq 2$ be an integer. Then, $h(G) \leq k$ if and only if $E U_{k}(G) \neq \emptyset$ where $E U_{k}(G)$ denotes the set of those subgraphs $H$ of $G$ which satisfy the following five conditions:

(I) $H$ is an even subgraph;

(II) $V_{0}(H) \subseteq \bigcup_{i=3}^{\Delta(G)} V_{i}(G) \subseteq V(H)$

(III) $d_{G}\left(H_{1}, H-H_{1}\right) \leq k-1$ for every induced subgraph $H_{1}$ of $H$ with $\emptyset \neq V\left(H_{1}\right) \subset$ $V(H)$

$(I V)|E(B)| \leq k+1$ for every branch $B \in \mathcal{B}(G) \backslash \mathcal{B}_{H}(G)$;

$(V)|E(B)| \leq k$ for every branch $B$ in $\mathcal{B}_{1}(G)$.

Note that, if we only consider 2-connected graphs, then Condition (V) in the definition of $E U_{k}(G)$ is superfluous. 


\subsection{Proof of Theorem 4.1.3}

Before presenting our proof, we start by proving three useful lemmas.

Lemma 4.2.1 Let $G^{\prime}$ be a reduced graph such that $\kappa^{\prime}\left(G^{\prime}\right) \geq 2$ and $\alpha\left(G^{\prime}\right) \leq 3$, then $\kappa\left(G^{\prime}\right) \geq 2$.

Proof of Lemma 4.2.1. Assume, in contrast, that $v$ is a cut vertex of $G^{\prime}$, then $d_{G^{\prime}}(v) \geq 4$ by $\kappa^{\prime}\left(G^{\prime}\right) \geq 2$. Since $G^{\prime}$ is triangle-free, $v$ has at least four neighbors in $G^{\prime}$ which form an independent set of $G^{\prime}$, a contradiction.

Lemma 4.2.2 Let $G^{\prime}$ be a reduced graph such that $\kappa\left(G^{\prime}\right)=2$ and $\alpha\left(G^{\prime}\right)=3$. Then, exactly one of the following holds.

(a) $G^{\prime}$ is supereulerian;

(b) $G^{\prime}$ is isomorphic to one of the three graphs depicted in Figure 4.1.

Proof of Lemma 4.2.2. Let $k=\kappa\left(G^{\prime}\right)$, and $C=u_{1} u_{2} \cdots u_{1}$ be a closed trail with a maximal number of vertices in $C$ (i.e., $\left|V\left(G^{\prime}\right) \backslash V(C)\right|$ is minimal). Then, $C$ is a cycle (otherwise there is a vertex $v$ of $C$ with $d_{C}(v) \geq 4$ and hence the neighbors of $v$ consist of an independent set of at least four vertices of $G^{\prime}$ by Theorem 1.2.2, contradicting that $\left.\alpha\left(G^{\prime}\right)=3\right)$. We denote by $u^{+}\left(u^{-}\right.$, respectively) the successor (the predecessor, respectively) of $u$ on $C$, and let $u^{++}=\left(u^{+}\right)^{+}, u^{--}=\left(u^{-}\right)^{-}$and so on; a portion of $C$ from $u$ to $v$ means $u u^{+} u^{++} \ldots v^{-} v$. Since $G^{\prime}$ is triangle-free, we obtain that $u^{-} u^{+} \notin E(G)$ for any vertex $u$ in $C$. Since $\delta\left(G^{\prime}\right) \geq \kappa\left(G^{\prime}\right)$, and every graph with $\delta\left(G^{\prime}\right) \geq 2$ has a cycle of length at least $\delta\left(G^{\prime}\right)+1, C$ has at least $k+1$ vertices.

If $C$ is not a spanning cycle of $G^{\prime}$, then there is a component $H$ of $G^{\prime}-V(C)$. We will prove that $G^{\prime}$ is isomorphic to one of those graphs depicted in Figure 4.1.

Since $\kappa\left(G^{\prime}\right)=2, C$ has at least two vertices having neighbors in $V(H)$. Let $x, y$ be any two vertices of $C$ having neighbors in $V(H)$. 
We claim that none of $\left\{x^{-}, x^{+}, y^{-}, y^{+}\right\}$has a neighbor in $H$; otherwise, say, $x^{+}$has a neighbor in $H$, then we can obtain a cycle from $C$ by adding an $x, x^{+}$-path through $H$ and by deleting the edge $x x^{+}$, which contains more vertices than $C$, contradicting the maximality of $|V(C)|$. We have the following two facts.

Claim 1. $x^{+} y^{+} \notin E\left(G^{\prime}\right)$ and $x^{-} y^{-} \notin E\left(G^{\prime}\right)$.

Proof of Claim 1. By symmetry, we only need to prove that $x^{+} y^{+} \notin E\left(G^{\prime}\right)$. Since $C$ is a cycle and $x \neq y^{+}, x^{+} y^{+} \notin E(C)$. Suppose $x^{+} y^{+} \in E\left(G^{\prime}\right) \backslash E(C)$, then we can obtain a cycle that contains more vertices than $C$ by including $x^{+} y^{+}$, the portions of $C$ from $x^{+}$to $y$ and from $y^{+}$to $x$, and an $x, y$-path through $H$, contradicting the maximality of $|V(C)|$.

Claim 2. Either $x^{+}=y^{-}$or $x^{+} y^{-} \in E(C)$ and either $x^{-}=y^{+}$or $x^{-} y^{+} \in E(C)$.

Proof of Claim 2. By symmetry, we only need to prove the second part of the claim. We will prove this by contradiction. If possible, suppose that neither $x^{-}=y^{+}$ nor $x^{-} y^{+} \in E(C)$, then there are at least five vertices in the portion of $C$ from $y$ to $x$. Suppose first that $x^{-} y^{+} \notin E\left(G^{\prime}\right)$; then, by Claim $1, S=\left\{x^{+}, x^{-}, y^{+}\right\}$is an independent set such that none of $S$ is adjacent to $H$, hence we can get an independent set of four vertices by adding one vertex of $H$ to $S$, contradicting $\alpha\left(G^{\prime}\right)=3$. Now, suppose that $x^{-} y^{+} \in E\left(G^{\prime}\right)$; then, by the fact that $G^{\prime}$ is triangle-free and by the assumption that $x^{-} y^{+} \notin E(C), x^{--} y^{+} \notin E\left(G^{\prime}\right)$ and there are at least six vertices in the portion of $C$ from $y$ to $x$. Note that $x^{--}$is not adjacent to any vertex of $H$; otherwise we can obtain a cycle that contains more vertices than $C$ by including $x^{-} y^{+}$, the portions of $C$ from $y^{+}$to $x^{--}$and from $x^{-}$to $y$, and an $x^{--}, y$-path through $H$, contradicting $\alpha\left(G^{\prime}\right)=3$. Hence, $x^{--} x^{+} \in E\left(G^{\prime}\right)$; otherwise $\left\{x^{+}, x^{--}, y^{+}, h\right\}$ is an independent set of four vertices, where $h \in V(H)$, contradicting $\alpha\left(G^{\prime}\right)=3$. Note that $x^{--} x^{+} \notin E(C)$. Hence the cycle obtained from $C$ by deleting three edges $x x^{+}, x^{--} x^{-}, y y^{+}$, and by adding two edges $x^{+} x^{--}, y^{+} x^{-}$ and an $x, y$-path through $H$, contains more vertices than $C$, contradicting the maximality of $|V(C)|$. This proves Claim 2.

By Claim 2, $C$ is a cycle of length at most six and any vertex in $V(C) \backslash\{x, y\}$ is not adjacent to $H$. This implies that $\{x, y\}$ is a 2-vertex cut-set of $G^{\prime}$. By the choice of $C$, 
$H$ has at most two vertices; otherwise, suppose $H$ has at least three vertices, then $x$ and $y$ must have different neighbors $h_{x}$ and $h_{y}$ in $H$, respectively, since $\kappa\left(G^{\prime}\right)=2$. No matter whether $h_{x}$ and $h_{y}$ are adjacent or not, there must be a path $P\left(h_{x}, h_{y}\right)$ of $H$ between $h_{x}$ and $h_{x}$ with length at least two, since $\kappa\left(G^{\prime}\right)=2$. Hence, we will obtain a longer cycle than $C$ by including the path $P\left(h_{x}, h_{y}\right), x h_{x}, y h_{y}$ and a longest portion of $C$ between $x$ and $y$, contradicting the maximality of $|V(C)|$. So $|V(H)| \leq 2$.

Suppose first that $|V(C)|=6$; then by Claim 2, we may assume $C=x x^{+} y^{-} y y^{+} x^{-} x$. Then $|V(H)|=1$; otherwise, since $\kappa\left(G^{\prime}\right)=2,|V(H)|=2$ and $G^{\prime}$ is triangle-free, we may assume that $V(H)=\left\{h_{x}, h_{y}\right\}$ with $x h_{x}, y h_{y} \in E\left(G^{\prime}\right)$ and $x h_{y} \notin E\left(G^{\prime}\right)$; and hence $\left\{x, y^{-}, y^{+}, h_{y}\right\}$ is an independent set of $G^{\prime}$, contradicting $\alpha\left(G^{\prime}\right)=3$. Hence, $x$ and $y$ share the same neighbor in $H$. It follows that $x y \notin E\left(G^{\prime}\right)$ since $G^{\prime}$ is triangle-free. So, $C$ is an induced cycle of $G^{\prime}$, by the fact that $G^{\prime}$ is triangle-free and by Claims 1 and 2 . Hence, $G^{\prime}$ is the graph $K_{2,3}(2)$ depicted in Figure 4.1. Next, suppose that $|V(C)|=4$ or 5 ; then $|V(H)|=1$ (otherwise, since $\kappa\left(G^{\prime}\right)=2$ and $|V(H)|=2$, we may assume that $V(H)=\left\{h_{x}, h_{y}\right\}$ with $x h_{x}, y h_{y}, h_{x} h_{y} \in E\left(G^{\prime}\right)$ and obtain a longer cycle than $C$ by including the path $x h_{x} h_{y} y$ and a longest portion of $C$ between $x$ and $y$, contradicting the maximality of $|V(C)|)$. Since $G^{\prime}$ is triangle-free, $x y \notin E\left(G^{\prime}\right)$. It follows that $C$ is an induced cycle of $G^{\prime}$, by Claims 1 and 2. Note that the vertex of $H$ is not adjacent to any vertex of $V(C) \backslash\{x, y\}$. Hence, if $|V(C)|=5$, then $G^{\prime}$ is the graph $K_{2,3}(1)$ depicted in Figure 4.1; if $|V(C)|=4$, then $G^{\prime}=K_{2,3}$. Above all, $G^{\prime}$ is isomorphic to one of those graphs depicted in Figure 4.1. This completes the proof of Lemma 4.2.2.

Lemma 4.2.3 Let $G^{\prime}$ be the reduction of a simple graph $G$. Then, the following statements hold.

(a) If $v$ is a nontrivial vertex of $G^{\prime}$ with $d_{G^{\prime}}(v)=2$, then there exists at least one vertex $v^{\prime}$ of $R_{G^{\prime}}^{-}(v)$ such that $v^{\prime}$ is not adjacent to any vertex of $\bigcup_{s \in V\left(G^{\prime}\right) \backslash\{v\}} R_{G^{\prime}}^{-}(s)$;

(b) If $u$ is a nontrivial vertex of $G^{\prime}$ with $d_{G^{\prime}}(u)=3$, then for any pair of vertices $\{x, y\} \subseteq N_{G^{\prime}}(u)$, there exists a least one vertex $u^{\prime}$ of $R_{G^{\prime}}^{-}(u)$ such that $u^{\prime}$ is not adjacent to any vertex of $R_{G^{\prime}}^{-}(x) \cup R_{G^{\prime}}^{-}(y)$. 
Proof of Lemma 4.2.3. Note that, by the definition of contraction, $R_{G^{\prime}}^{-}(s)$ is collapsible for any nontrivial vertex $s$ of $V\left(G^{\prime}\right)$; hence, since $G$ is simple, $R_{G^{\prime}}^{-}(s)$ has at least three vertices. Therefore, since $d_{G^{\prime}}(v)=2$, there are exactly two vertices $v^{\prime \prime}, v^{\prime \prime \prime}$ of $R_{G^{\prime}}(v)$ that are adjacent to some vertex of $\bigcup_{s \in N_{G^{\prime}} \backslash\{v\}} R_{G^{\prime}}^{-}(s)$ by the definition of contraction. Hence, there exists at least one vertex $v^{\prime} \in R_{G^{\prime}}(v) \backslash\left\{u^{\prime \prime}, u^{\prime \prime \prime}\right\}$ that is not adjacent to any vertex of $\bigcup_{s \in V\left(G^{\prime}\right) \backslash\{v\}} R_{G^{\prime}}^{-}(s)$. This proves the first statement. For a nontrivial vertex $u$ of $G^{\prime}$ with $d_{G^{\prime}}(u)=3$, there is exactly one vertex of $R_{G^{\prime}}^{-}(u)$ that is adjacent to some vertex of $R_{G^{\prime}}^{-}(s)$ for any $s \in N_{G^{\prime}}(u)$ by the definition of contraction. Therefore, since $\left|V\left(R_{G^{\prime}}^{-}(s)\right)\right| \geq 3$ for any nontrivial vertex $s$ of $G^{\prime}$, the second statement holds. This completes the proof of Lemma 4.2.3.

Now we finish the proof of Theorem 4.1.3.

Proof of Theorem 4.1.3. Let $G^{\prime}$ be the reduction of $G$. If $G^{\prime}=K_{1}$, then $G$ is supereulerian by Theorem 1.2.2. So, we only need to consider the case when $G^{\prime}$ is not $K_{1}$.

If $\kappa(G) \geq 4$, then $G$ is supereulerian by Theorem 4.1.7.

If $\kappa(G)=3$ then, by hypothesis, we have that $\alpha(G) \leq 4$. We let $\alpha(G)=4$ since, otherwise, $G$ is hamiltonian by Theorem 4.1.1. Theorem 4.1.10 gives that $\alpha\left(G^{\prime}\right) \leq 4$ and $\kappa^{\prime}\left(G^{\prime}\right) \geq \kappa^{\prime}(G) \geq \kappa(G) \geq 3$. First, suppose that $\alpha\left(G^{\prime}\right)=4$, then from Theorem 4.1.8 we deduce that $\left|V\left(G^{\prime}\right)\right| \leq 11$. Hence, $G^{\prime}$ is either collapsible or the Petersen graph by Theorem 4.1.9. If $G^{\prime}$ is collapsible, then $G^{\prime}$ is supereulerian and $G$ is hence supereulerian by Theorem 1.2.2. If $G^{\prime}$ is the Petersen graph, then we claim that every vertex of $G^{\prime}$ is trivial. Assume, in contrast, that there exists a nontrivial vertex $w$ of $G^{\prime}$. Let $N_{G^{\prime}}(w)=$ $\{x, y, z\}$. By Lemma 4.2.3 (b), there exists at least one vertex $w^{\prime} \in R_{G^{\prime}}^{-}(w)$ such that $w^{\prime}$ is not adjacent to any vertex of $\cup_{v \in\{x, y\}} R_{G^{\prime}}^{-}(v)$. Let $N_{G^{\prime}}(z) \backslash\{w\}=\{u, v\}$. Taking $x^{\prime} \in R_{G^{\prime}}^{-}(x), y^{\prime} \in R_{G^{\prime}}^{-}(y), u^{\prime} \in R_{G^{\prime}}^{-}(u)$ and $v^{\prime} \in R_{G^{\prime}}^{-}(v)$, we obtain an independent set $\left\{w^{\prime}, x^{\prime}, y^{\prime}, u^{\prime}, v^{\prime}\right\}$ of $G$, contradicting that $\alpha(G)=4$. This shows that $G^{\prime}$ has no nontrivial vertex. Hence, $G=G^{\prime}$, i.e., $G$ is the Petersen graph. Now, suppose $\alpha\left(G^{\prime}\right) \leq 3$. Since $\kappa^{\prime}\left(G^{\prime}\right) \geq 3, \kappa\left(G^{\prime}\right) \geq 2$ by Lemma 4.2.1. In the case when $\kappa\left(G^{\prime}\right) \geq \alpha\left(G^{\prime}\right)$, we have that $G^{\prime}$ is hamiltonian (hence supereulerian) by Theorems 4.1.1. So $G$ is supereulerian by Theorem 1.2.2. It remains the case when $\kappa\left(G^{\prime}\right)=2$ and $\alpha\left(G^{\prime}\right)=3$. By Lemma 4.2.2, $G^{\prime}$ is isomorphic to one of the three graphs in Figure 4.1, which contradicts that $\kappa^{\prime}\left(G^{\prime}\right) \geq 3$. 
It remains to consider the case that $\kappa(G)=2$ and $\alpha(G)=3$. Theorem 4.1 .10 gives that $\alpha\left(G^{\prime}\right) \leq 3$ and $\kappa^{\prime}\left(G^{\prime}\right) \geq \kappa^{\prime}(G) \geq \kappa(G)=2$. Hence, $\kappa\left(G^{\prime}\right) \geq 2$ by Lemma 4.2.1. We only need to consider the case that $\kappa\left(G^{\prime}\right)=2$ and $\alpha\left(G^{\prime}\right)=3$ since, in all other cases, $G^{\prime}$ is hamiltonian and $G$ is hence supereulerian. By Lemma 4.2.2, either $G^{\prime}$ is supereulerian or $G^{\prime}$ is isomorphic to one of $\left\{K_{2,3}, K_{2,3}(1), K_{2,3}(2)\right\}$. If $G^{\prime}$ is supereulerian, then so is $G$ by Theorem 1.2.2.

Now, suppose that $G^{\prime}$ is isomorphic to one of $\left\{K_{2,3}, K_{2,3}(1), K_{2,3}(2)\right\}$. Then, we claim that $G^{\prime}$ has at most one nontrivial vertex. Assume, in contrast, that $G^{\prime}$ has two nontrivial vertices $u$ and $v$. Note that $G$ is a simple graph. Firstly, suppose $d_{G^{\prime}}(u)=d_{G^{\prime}}(v)=2$. Then, by Lemma 4.2.3 (a), there exist at least two vertices $u^{\prime}$ and $v^{\prime}$ in $R_{G^{\prime}}^{-}(u)$ and $R_{G^{\prime}}^{-}(v)$, respectively, such that they are not adjacent to any vertex of $\bigcup_{x \in V\left(G^{\prime}\right) \backslash\{u, v\}} R_{G^{\prime}}^{-}(x)$. Taking any pair of vertices $y^{\prime}$ in $R_{G^{\prime}}^{-}(y)$ and $z^{\prime}$ in $R_{G^{\prime}}^{-}(z)$ for the two vertices $y$ and $z$ of degree 3 in $G^{\prime}$, we obtain that $\left\{u^{\prime}, v^{\prime}, x^{\prime}, y^{\prime}\right\}$ is an independent set of $G$, contradicting $\alpha(G)=3$. Secondly, suppose $d_{G^{\prime}}(u)=d_{G^{\prime}}(v)=3$, and $x, y$ are vertices with degree two in $G^{\prime}$ and $x y \notin E\left(G^{\prime}\right)$. Then, by Lemma 4.2 .3 (b), there exist at least two vertices $x^{\prime}$ and $y^{\prime}$ in $R_{G^{\prime}}^{-}(x)$ and $R_{G^{\prime}}^{-}(y)$, respectively, such that they are not adjacent to any of $R_{G^{\prime}}^{-}(u) \cup R_{G^{\prime}}^{-}(v)$. It follows that $\left\{x^{\prime}, y^{\prime}, u^{\prime}, v^{\prime}\right\}$ is an independent set of $G$ (where $u^{\prime} \in R_{G^{\prime}}^{-}(u$ ) and $\left.v^{\prime} \in R_{G^{\prime}}^{-}(v)\right)$, contradicting $\alpha(G)=3$. Finally, suppose that $d_{G^{\prime}}(u) \neq d_{G^{\prime}}(v)$, say, $d_{G^{\prime}}(u)=3, d_{G^{\prime}}(v)=2$. Take a pair of vertices $\{x, y\} \subseteq V_{2}\left(G^{\prime}\right) \backslash\{v\}$ such that $x y \notin E\left(G^{\prime}\right)$. Hence, by Lemma $4.2 .3(\mathrm{~b})$, there exists at least one vertex $u^{\prime}$ (say) of $R_{G^{\prime}}^{-}(u)$ that is not adjacent to any vertex of $R_{G^{\prime}}^{-}(x) \cup R_{G^{\prime}}^{-}(y)$, and there is a vertex $v^{\prime}$ of $R_{G^{\prime}}^{-}(v)$ that is not adjacent to any vertex of $\bigcup_{s \in V\left(G^{\prime}\right) \backslash\{v\}} R_{G^{\prime}}^{-}(s)$. Taking $x^{\prime} \in R_{G^{\prime}}^{-}(x)$ and $y^{\prime} \in R_{G^{\prime}}^{-}(y)$, we obtain an independent set $\left\{x^{\prime}, y^{\prime}, v^{\prime}, u^{\prime}\right\}$ of $G$, contradicting $\alpha(G)=3$. This proves that $G^{\prime}$ has at most one nontrivial vertex if $G^{\prime} \in\left\{K_{2,3}, K_{2,3}(1), K_{2,3}(2)\right\}$.

If $G^{\prime}$ has no nontrivial vertices, then $G=G^{\prime}$ and $G$ is isomorphic to one of those graphs in Figure 4.1. Now, suppose that $G^{\prime}$ has a nontrivial vertex $u$. Note that $G$ is a simple graph. Hence, if $d_{G^{\prime}}(u)=2$, then, by Lemma 4.2.3 (a), there is at least one vertex $u^{\prime}$ of $R_{G^{\prime}}^{-}(u)$ such that $u^{\prime}$ is not adjacent to any vertex in $\bigcup_{x \in V\left(G^{\prime}\right) \backslash\{u\}} R_{G^{\prime}}^{-}(x)=V\left(G^{\prime}\right) \backslash\{u\}$; if $d_{G^{\prime}}(u)=3$ then, by Lemma $4.2 .3(\mathrm{~b})$, for any pair of vertices in $N_{G^{\prime}}(u)$, say $s$ and $t$, there exists a vertex in $R_{G^{\prime}}^{-}(u)$ which is adjacent to neither $s$ nor $t$, since $\left|V\left(R_{G^{\prime}}^{-}(u)\right)\right| \geq 3$. Hence, $G^{\prime}=K_{2,3}(2)$ has no nontrivial vertices. For $G^{\prime} \in\left\{K_{2,3}, K_{2,3}(1)\right\}, R_{G^{\prime}}^{-}(u)$ must be 
a complete graph. Suppose, in contrast, that there exist two nonadjacent vertices $s, t$ in $R_{G^{\prime}}^{-}(u)$. If $d_{G^{\prime}}(u)=2$, then the vertices $s, t$ and the other two trivial vertices (that are not adjacent to $u$ in $G^{\prime}$ ) of degree two in $G^{\prime}$ induce an independent set in $G$, contradicting $\alpha(G)=3$. If $d_{G^{\prime}}(u)=3$, then there exists one vertex in $R_{G^{\prime}}^{-}(u)$ that is not adjacent to any one of the three independent trivial vertices of degree two in $G^{\prime}$, contradicting $\alpha(G)=3$. Hence, $R_{G^{\prime}}^{-}(u)$ is a complete graph. If $G^{\prime}=K_{2,3}$ and $d_{G^{\prime}}(u)=3$, then $R_{G^{\prime}}^{-}(u)$ is a triangle and the original graph $G$ is isomorphic to $K_{2,3}^{\prime}$ (otherwise, in the way similar to the proof of Lemma 4.2.3 (b), we can prove that there exists a vertex in $R_{G^{\prime}}^{-}(u)$ that is not adjacent to any one of the three nonadjacent vertices of degree two in $G^{\prime}$, contradicting $\alpha(G)=3$ ). If $G^{\prime}=K_{2,3}(1)$, then $d_{G^{\prime}}(u)=2$ (otherwise, by Lemma 4.2 .3 (b) and by the fact that there exists a vertex of degree two that is not adjacent to $u$, there exists a vertex in $R_{G^{\prime}}^{-}(u)$ that is not adjacent to any one of the three independent vertices of degree two in $G^{\prime}$, contradicting $\alpha(G)=3)$. Note that there exists a vertex $w$ of $R_{G^{\prime}}^{-}(u)$ such that it is not adjacent to any vertex of $V\left(G^{\prime}\right) \backslash\{u\}\left(=\bigcup_{z \in V\left(G^{\prime}\right) \backslash\{u\}} R_{G^{\prime}}^{-}(z)\right)$. We have that any vertex of $N_{G^{\prime}}(u)$ has degree three in $G^{\prime}$ (otherwise $\{w\} \bigcup\left(V_{2}\left(G^{\prime}\right) \backslash\{u\}\right)=\{w\} \bigcup\left(\bigcup_{z \in V_{2}\left(G^{\prime}\right) \backslash\{u\}} R_{G^{\prime}}^{-}(z)\right)$ is an independent set of four vertices in $G$, contradicting $\alpha(G)=3$ ). This completes the proof of Theorem 4.1.3.

\subsection{Proof of Theorem 4.1.6}

Before presenting our proof of Theorem 4.1.6, we need some additional terminology and notation. A subset $S$ of $\mathcal{B}(G)$ is called a branch cut if the deletion of all edges and all inner vertices (of degree two) in any branch of $S$ will induce more components than $G$ has. A minimal branch cut is called a branch-bond. It is easily shown that, for a connected graph $G$, a subset $S$ of $\mathcal{B}(G)$ is a branch-bond if and only if the deletion of all edges and all inner vertices (of degree two) in any branch of $S$ will induce exactly two components. We denote by $\mathcal{B B}(G)$ the set of branch-bonds of $G$. A branch-bond $S \in \mathcal{B B}(G)$ is called odd if $S$ consists of an odd number of branches. The length of a branch-bond $S \in \mathcal{B B}(G)$, denoted by $l(S)$, is the length of a shortest branch in it. Define $\mathcal{B B}_{3}(G)=\{S \in \mathcal{B B}(G):|S| \geq 3$ and $S$ is odd $\}$. Define $h_{3}(G)=\max \{l(S): S \in$ $\left.\mathcal{B B}_{3}(G)\right\}$ if $\mathcal{B B}_{3}(G)$ is not empty; 0 , otherwise. Note that, if $H$ is an even graph of $G$ and 
$C$ is a cycle of $G$, then $G[E(H) \triangle E(C)]$ is also an even graph of $G$, but $G[E(H) \triangle E(C)]$ may have more components than $H$.

From a result of [48], one can easily obtain the following.

Theorem 4.3.1 (Xiong and Wu, [49]) Let $G$ be a 2-connected graph. Then,

$$
h_{3}(G)-1 \leq h(G) \leq h_{3}(G)+1
$$

The following characterization of eulerian graphs involves branch-bonds.

Theorem 4.3.2 (Xiong, Broersma, Li and Li, [48]) A connected graph is eulerian if and only if each branch-bond contains an even number of branches.

Now, we present the proof of our second main result.

Proof of Theorem 4.1.6. By Theorems 4.1.1 and 4.1.4, we only need to consider the case when $t \geq 2$. Let $m=\left\lfloor\frac{2 t+2}{3}\right\rfloor$. Then, $m \geq 2$. Since $h(G) \leq 2$ for the graph $G$ with $\kappa(G) \geq 3$ by Theorem 4.1.11, we can assume that $\kappa(G)=2$. If $h_{3}(G) \leq m-1$, then $h(G) \leq m$ by Theorem 4.3.1. So, it remains to consider the case when $h_{3}(G) \geq m$. Let $\mathcal{B}_{0}$ be a branch-bond in $\mathcal{B B}_{3}(G)$ such that $h_{3}(G)=\min \left\{|E(B)|: B \in \mathcal{B}_{0}\right\}$. Note that there are at least $\left\lfloor\frac{3 h_{3}(G)}{2}\right\rfloor$ vertices in $G\left[\cup_{B \in \mathcal{B}_{0}} V(B)\right]$ consisting of an independent set of $G$, which contains at most one end vertex of $\mathcal{B}_{0}$, and hence $\alpha(G) \geq\left\lfloor\frac{3 h_{3}(G)}{2}\right\rfloor$. We claim that $h_{3}(G) \leq m+1$ since otherwise $\alpha(G) \geq\left\lfloor\frac{3 h_{3}(G)}{2}\right\rfloor \geq \frac{3 h_{3}(G)-1}{2} \geq \frac{3 m+5}{2}$, which contradicts the fact that

$$
\alpha(G) \leq \kappa(G)+t=2+t<2+\frac{3 m+3-2}{2}=\frac{3 m+5}{2} .
$$

We will prove that there is a subgraph $H \in E U_{m}(G)$ that implies that $h(G) \leq m=\left\lfloor\frac{2 t+2}{3}\right\rfloor$ by Theorem 4.1.11.

Since the edgeless graph with vertex set $\bigcup_{i=3}^{\Delta(G)} V_{i}(G)$ satisfies (I) and (II), we may let $H$ be a subgraph such that

(1) $H$ satisfies (I) and (II); 
(2) subject to (1), $H$ contains a maximum number of branches of length at least $m+2$;

(3) subject to (1) and (2), $\max _{\emptyset \neq V\left(H_{1}\right) \subset V(H)} d_{G}\left(H_{1}, H-H_{1}\right)$ is minimized;

(4) subject to the above, $H$ contains a minimum number of induced subgraphs $F$ for which $d_{G}(F, H-F)=\max _{\emptyset \neq V\left(H_{1}\right) \subset V(H)} d_{G}\left(H_{1}, H-H_{1}\right)$.

We claim that $H \in E U_{m}(G)$. It suffices to prove that $H$ satisfies (III) and (IV) since $G$ is 2-connected. It suffices to prove the following two claims.

Claim 3. $d_{G}\left(H_{1}, H-H_{1}\right) \leq m-1$ for any subgraph $H_{1}$ of $H$.

Proof of Claim 3. Assume, in contrast, that there is an induced subgraph $F$ of $H$ with $d_{G}(F, H-F) \geq m \geq 2$, then any shortest path between $F$ and $H-F$ is a branch of $G$. Since $G$ is 2-connected, there are at least two branches $P_{1}, P_{2}$ between $F$ and $H-F$ and we may hence choose a cycle $C$ containing both $P_{1}$ and $P_{2}$ such that the length of a longest branch in $\mathcal{B}_{C}(G) \cap \mathcal{B}_{H}(G)$ is as small as possible. Hence, $\left|E\left(P_{i}\right)\right| \geq m$. By the choice of $C$, we have the fact that if $B$ is the longest branch in $\mathcal{B}_{C}(G) \cap \mathcal{B}_{H}(G)$, then there exists at least one branch $B^{\prime} \in \mathcal{B}_{H}(G) \backslash \mathcal{B}_{C}(G)$ of length at least $|E(B)|$ such that the end vertices of both $B$ and $B^{\prime}$ are all in the same one of $\{H, H-F\}$. We claim that either there are at least two branches of length at least $m+2$ such that all of their end vertices belong to the same one of $\{F, H-F\}$, or there are at least two branches $B_{1}, B_{2}$ of length $m$ or $m+1$ that are in $\mathcal{B}(G) \backslash\left\{P_{1}, P_{2}\right\}$ such that $E\left(B_{i}\right) \in E(C)$ and all end vertices of both $B_{1}$ and $B_{2}$ are in the same one of $\{F, H-F\}$ (otherwise the subgraph $H^{\prime}$ obtained from $G[E(H) \triangle E(C)]$ by adding the remaining vertices of $\bigcup_{i=3}^{\Delta(G)} V_{i}(G)$ as isolated vertices of $H^{\prime}$ is a subgraph satisfying (1) and (2) such that either $\max _{\mathfrak{\emptyset} \neq V\left(H_{1}\right) \subset V\left(H^{\prime}\right)} d_{G}\left(H_{1}, H^{\prime}-H_{1}\right)$ is less than $\max _{\mathfrak{\emptyset} \neq V\left(H_{1}\right) \subset V(H)} d_{G}\left(H_{1}, H-H_{1}\right)$ or $H^{\prime}$ has less induced subgraphs $F$ for which $d_{G}\left(F, H^{\prime}-F\right)=\max _{\mathscr{Q} \neq V\left(H_{1}\right) \subset V\left(H^{\prime}\right)} d_{G}\left(H_{1}, H^{\prime}-H_{1}\right)$ than $H$ (in this case, $H^{\prime}$ satisfies (3)), which shows that $H$ does not satisfy (3) or (4), a contradiction). Since $G$ is 2-connected, every component of $H$ has at least one vertex which is not adjacent to any inner vertex of branches of length at least $m$ in $\mathcal{B}_{C}(G)$. Hence, in the first case, there exist at least $\left\lfloor\frac{m+2}{2}\right\rfloor+2\left\lfloor\frac{m}{2}\right\rfloor$ inner vertices in the four branches consisting of an independent set and hence $\alpha(G) \geq 2\left\lfloor\frac{m+2}{2}\right\rfloor+2\left\lfloor\frac{m}{2}\right\rfloor+1 \geq \frac{3 m+5}{2}$, which contradicts (4.1). In the second case, by the definition of odd branch-bonds, there are at least two branches of length $m$ or $m+1$ 
in $\mathcal{B}(G) \backslash \mathcal{B}_{0}$. Hence, there are at least $2\left\lfloor\frac{m}{2}\right\rfloor+1 \geq 3$ vertices consisting of an independent set, any element of which is not adjacent to any inner vertex of any branch of $\mathcal{B}_{0}$, and so $\alpha(G) \geq\left\lfloor\frac{3 h_{3}(G)}{2}\right\rfloor+3 \geq \frac{3 m-1}{2}+3 \geq \frac{3 m+5}{2}$, which contradicts (4.1). This proves Claim 3 .

Claim 4. $|E(B)| \leq m+1$ for any branch $B \in \mathcal{B}(G) \backslash \mathcal{B}_{H}(G)$.

Proof of Claim 4. Assume, in contrast, that there is a branch $B_{0} \in \mathcal{B}(G) \backslash \mathcal{B}_{H}(G)$ with $\left|E\left(B_{0}\right)\right| \geq m+2$. Let $u$ and $v$ be two end vertices of $B_{0}$ and $S\left(u, B_{0}\right)$ be a branchbond containing $B_{0}$ such that any branch of $S\left(u, B_{0}\right)$ has $u$ as an end vertex. Since $G$ is 2-connected, $\left|S\left(u, B_{0}\right)\right| \geq 2$.

By the following algorithm, we first find a cycle of $G$ that contains $B_{0}$ and then obtain a contradiction.

\section{Algorithm $B_{0}$.}

1. If $\left|S\left(u, B_{0}\right)\right|$ is even, then select a branch $B_{1} \in S\left(u, B_{0}\right) \backslash\left(\mathcal{B}_{H}(G) \cup\left\{B_{0}\right\}\right)$ by Theorem 4.3.2. Otherwise, since $\left|E\left(B_{0}\right)\right| \geq m+2$, (we can) select a branch $B_{1} \in S\left(u, B_{0}\right)$ with

$$
\left|E\left(B_{1}\right)\right|=l\left(S\left(u, B_{0}\right)\right) \leq h_{3}(G) \leq m+1
$$

(obviously $\left.B_{1} \neq B_{0}\right)$ and let $u_{1}(\neq u)$ be the other end vertex of $B_{1}$. If $u_{1}=v$, then set $t:=1$ and stop. Otherwise $i:=1$.

2. Select a branch-bond $S\left(u, u_{i}, B_{0}\right)$ in $G$ which contains $B_{0}$ but not any of $\left\{B_{1}, B_{2}, \cdots, B_{i}\right\}$ such that any branch in $S\left(u, u_{i}, B_{0}\right)$ has exactly one end vertex in $\left\{u, u_{1}, u_{2}, \cdots, u_{i}\right\}$. If $\left|S\left(u, u_{i}, B_{0}\right)\right|$ is even, then, by Theorem 4.3 .2 , (we can) select a branch

$$
B_{i+1} \in S\left(u, u_{i}, B_{0}\right) \backslash\left(\mathcal{B}_{H}(G) \cup\left\{B_{0}\right\}\right) .
$$

Otherwise, since $\left|E\left(B_{0}\right)\right| \geq h_{3}(G)+1$, (we can) select a branch $B_{i+1} \in S\left(u, u_{i}, B_{0}\right)$ such that

$$
\left|E\left(B_{i+1}\right)\right|=l\left(S\left(u, u_{i}, B_{0}\right)\right) \leq h_{3}(G)
$$

(obviously $B_{i+1} \neq B_{0}$ ), and let $u_{i+1}$ be the end vertex of $B_{i+1}$ that is not in $\left\{u, u_{1}, u_{2}, \cdots, u_{i}\right\}$.

3. If $u_{i+1}=v$, then set $t:=i+1$ and stop. Otherwise replace $i$ by $i+1$ and return to step 2 . 
Note that $|\mathcal{B}(G)|$ is finite, and $d_{G}(v) \geq 2$ implies that Algorithm $B_{0}$ will stop after a finite number of steps. Note that $G\left[\bigcup_{i=0}^{t} E\left(B_{i}\right)\right]$ is connected. Furthermore, since $u_{t}=v$ and $\left|S\left(u, u_{i}, B_{0}\right)\right| \geq 2, G\left[\bigcup_{i=0}^{t} E\left(B_{i}\right)\right]$ has a cycle $C_{0}$ of $G$ which contains $B_{0}$. Let $H^{\prime}$ be the subgraph of $G$ obtained from $G\left[E(H) \triangle E\left(C_{0}\right)\right]$ by adding the remaining vertices of $\bigcup_{i=3}^{\Delta(G)} V_{i}(G)$ as isolated vertices in $H^{\prime}$. By the choice of $B_{i},|E(B)| \leq h_{3}(G) \leq$ $m+1$ for $B \in \mathcal{B}_{H}(G) \cap\left\{B_{1}, B_{2}, \cdots, B_{t}\right\}$. Hence, since $B_{0}$ is in $\mathcal{B}_{C_{0}}(G), H^{\prime}$ is a subgraph with the conditions (I) and (II) that has less branches of length $m+2$ than $H$, which shows that $H$ does not satisfy (2), a contradiction. This proves Claim 4.

Thus we, in fact, have proved that $G$ has a subgraph in $E U_{m}(G)$ by Claims 3 and 4. Hence, $h(G) \leq m=\left\lfloor\frac{2 t+2}{3}\right\rfloor$ by Theorem 4.1.11. This also completes the proof of Theorem 4.1.6.

\section{Sharpness}

In this part, we discuss sharpness. We show that Theorem 4.1.6 is sharp by describing a graph satisfying the conditions of Theorem 4.1.6 and having the hamiltonian index of exactly $\left\lfloor\frac{2 t+2}{3}\right\rfloor$. By Theorems 4.1.1, 4.1.3 and 1.1.2, we only need to show the sharpness in the case when $t \geq 2$.

Let $H_{1}, H_{2}$ be two vertex-disjoint complete graphs of order at least four and $P_{1}, P_{2}, P_{3}$ be three vertex-disjoint paths with length $m+1 \geq 3$. Now, obtain the graph $G_{0}$ by identifying three end vertices of $P_{1}, P_{2}, P_{3}$ with three vertices of $H_{1}$, and identifying the other end vertex of $P_{i}$ with three vertices of $H_{2}$, respectively, see Figure 4.3.

Let $C$ be a longest cycle of $G_{0}$. Then, $C \in E U_{m}\left(G_{0}\right)$. So $h\left(G_{0}\right) \leq m$ by Theorem 4.1.11, and $h\left(G_{0}\right) \geq h_{3}\left(G_{0}\right)-1=m$ by Theorem 4.3.1. Hence, $h\left(G_{0}\right)=m$. Note that if $m$ is even, then $t=\alpha\left(G_{0}\right)-2=\left\lfloor\frac{3 m}{2}\right\rfloor$ and $h\left(G_{0}\right)=m=\left\lfloor\frac{2 t+2}{3}\right\rfloor$. For the case when $m$ is odd, consider the reduction $G_{0}^{\prime}$ of $G_{0}$, which is obtained from $G_{0}$ by contracting $H_{1}$ and $H_{2}$, respectively. Then $t=\alpha\left(G_{0}^{\prime}\right)-2=\left\lfloor\frac{3 m}{2}\right\rfloor$ and $h\left(G_{0}^{\prime}\right)=m=\left\lfloor\frac{2 t+2}{3}\right\rfloor$ for $m$ is odd. 
CHAPTER 4. THE CHVÁTAL-ERDÖS CONDITION FOR SUPEREULERIAN GRAPHS52

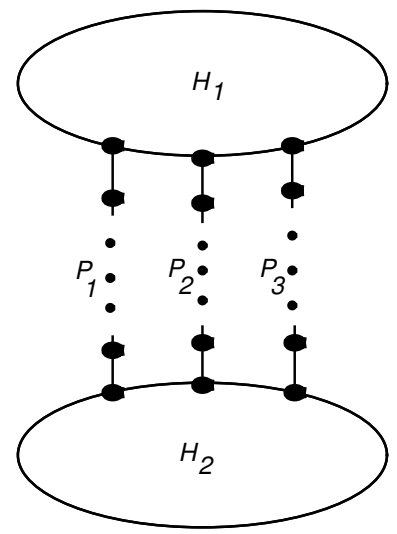

Figure 4.3: The graph $G_{0}$ 


\section{Chapter 5}

\section{2-factors in Line Graphs}

\subsection{Introduction}

A 2-factor is a 2-regular spanning subgraph of $G$. A hamiltonian cycle is then a 2-factor, and in one sense, it is the simplest 2-factor as it is composed of a single cycle. A circuit is a connected graph with at least three vertices in which every vertex has even degree. Let $F$ be a vertex subset of $V(G)$. An eulerian subgraph $H$ of $G$ is called $F$-eulerian subgraph if $F \subseteq V(H)$.

A star is the complete bipartite graph $K_{1, m}$. For a given graph $G$, we say that $G$ has a $k$-system that dominates if there is a family $\mathcal{S}$ of edge-disjoint circuits and stars with at least three edges in $G$ such that every edge of $G$ is either in one of the circuits or stars, or is incident with a circuit in $\mathcal{S}$, where $k=|\mathcal{S}|$. The following result gives a characterization of the graph $G$ such that its line graph $L(G)$ contains a 2-factor with exactly $k$ components.

Theorem 5.1.1 (Gould and Hynds, [27]) Let $G$ be a graph without isolated vertices. The line graph $L(G)$ contains a 2-factor with $k(k \geq 1)$ components if and only if $G$ has a $k$-system that dominates. 
Some recent results on 2-factors are in the following.

Theorem 5.1.2 (Chen et al., [19]) Let $G$ be a 2-connected, claw-free graph of order $n \geq 51$ with $\delta(G) \geq(n-2) / 3$, then for each $k$ with $1 \leq k \leq(n-24) / 3$, G has a 2-factor with exactly $k$ cycles.

Theorem 5.1.3 (Faudree et al., [25]) If $G$ is a 1-tough graph of sufficiently large order $n$ with $\delta(G) \geq(n-t) / 2,(0 \leq t \leq 4)$, then $G$ contains a 2-factor with $k$ cycles where $1 \leq k \leq n / 4-t$.

Theorem 5.1.4 (Niu and Xiong, [41]) Let $G$ be a connected simple graph of order $n$ and $k$ a positive integer such that $\delta(G) \geq\lfloor n / k\rfloor-1$. If $n$ is sufficiently large relative to $k$, then $G$ has an even factor with at most $k$ components, and then $L(G)$ has a 2-factor with at most $k$ components.

Conjecture 5.1.5 (Fujisawa et al., [26]) Let $G$ be a simple graph with $\delta(G) \geq 3$. Then $L(G)$ has a 2-factor with at most $\frac{n(2 \delta(G)-3)}{2\left(\delta(G)^{2}-\delta(G)-1\right)}$ components.

Catlin [8] showed the following:

Theorem 5.1.6 (Catlin, [8]) Let $G$ be a connected simple graph of order $n$, and let $p \geq 2$ be an integer. If $d(u)+d(v)>\frac{2 n}{p}-2$ whenever $u v \notin E(G)$, and if $n \geq 4 p^{2}$, then exactly one of the following conclusions holds:

(1) G has a spanning eulerian subgraph;

(2) $G$ is contractible to a graph $G_{1}$ of order less than $p$ and containing no spanning eulerian subgraph;

(3) $p=2$, and $G-x=K_{n-1}$ for some $x \in V(G)$ with $d(x)=1$.

Motivated by the above theorems, in this chapter, we are going to show the following main result, of which Theorem 5.1.4 is a special case. 
Theorem 5.1.7 Let $G$ be a connected simple graph of order $n$, and let $p$ be a positive integer, $\mu$ a nonnegative integer, $U=\left\{v: d(v)<\frac{n-\mu}{p}-1\right\}$. If $n>p^{3}+6 p^{2}+6 p+\mu p+\mu$, and if $\max \{d(x), d(y)\} \geq \frac{n-\mu}{p}-1$ holds for any $x y \notin E(G)$, then

(1) if $|U| \leq 1$, then $L(G)$ has a 2-factor with at most $p$ components;

(2) if $|U| \neq 2$, then $L(G)$ has a 2-factor with at most $p+1$ components.

Especially, if $\delta(G) \geq \frac{n}{p}-1$, i.e, $U=\emptyset$, then $L(G)$ has a 2-factor with at most $p$ components.

We will prove Theorem 5.1.7 in Section 5.2. Section 5.3 is devoted to some corollaries, and the sharpness of Theorem 5.1.7 is presented in the last section.

\subsection{Proof of Theorem 5.1.7}

Define

$$
J_{p}(G)=\left\{v \in V(G): d(v) \geq \frac{n-\mu}{p}-1\right\} .
$$

Let $G^{\prime}$ be the reduction of $G$ and $n^{\prime}=\left|V\left(G^{\prime}\right)\right|$. Let $p$ be a positive integer, and consider the condition that for any $x y \notin E(G)$,

$$
\max \{d(x), d(y)\} \geq \frac{n-\mu}{p}-1 .
$$

We shall assume that (5.1) holds and that $n$ is sufficiently large (say $n>p^{3}+6 p^{2}+6 p+$ $\mu p+\mu)$. Let $U=\left\{v: d(v)<\frac{n-\mu}{p}-1\right\}$. Then obviously we must have that $G[U]$ is a complete subgraph of $G$. Moreover, $d(v) \geq \frac{n-\mu}{p}-1$ for any $v \in V(G) \backslash U$.

Let $c=p+5$ and let

$$
W=\left\{v \in V\left(G^{\prime}\right): d_{G^{\prime}}(v) \leq c\right\} \text { and } W^{\prime}=\{v \in W: \quad v \text { is nontrivial }\} .
$$

We shall prove several claims to help us establish the conclusion of our main result. 
Claim $1\left|W \backslash W^{\prime}\right| \leq 1$.

Proof: Since every vertex $v$ of $W \backslash W^{\prime}$ is trivial, $d_{G}(v)=d_{G^{\prime}}(v) \leq c<\frac{n-\mu}{p}-1$ when $n>p^{2}+6 p+\mu$. Hence $W \backslash W^{\prime} \subseteq U$. Note that $|U| \neq 2$ and $G[U]$ is a complete subgraph of $G$. Thus $U \subseteq W^{\prime}$ if $|U| \geq 3$. This proves Claim 1 .

Claim 2 For any $v \in W^{\prime}$, if $H_{v}$ denotes the pre-image of $v$ in $G$ and either $|U| \leq 1$ or $G[U] \nsubseteq H_{v}$, then

$$
\left|V\left(H_{v}\right)\right| \geq \frac{n-\mu}{p}-d_{G^{\prime}}(v)
$$

Proof: Let $O u t\left(H_{v}\right)=\left\{x \in V\left(H_{v}\right): N_{G}(x) \neq N_{H_{v}}(x)\right\}$ and $\operatorname{In}\left(H_{v}\right)=V\left(H_{v}\right)-O u t\left(H_{v}\right)$. If there is an edge $x y \in E\left(H_{v}\right)$ such that $d(x) \geq d(y)$ and such that $x \in \operatorname{In}\left(H_{v}\right)$, since either $|U| \leq 1$ or $G[U] \nsubseteq H_{v},\left|V\left(H_{v}\right)\right| \geq d(x)+1 \geq \frac{n-\mu}{p}$, and so Claim 2 holds. Therefore, we assume that for any edge $x y \in E\left(H_{v}\right)$ with $d(x) \geq d(y)$, we always have $x \in \operatorname{Out}\left(H_{v}\right)$. Thus $\left|\operatorname{Out}\left(H_{v}\right)\right| \geq 1$. Since either $|U| \leq 1$ or $G[U] \nsubseteq H_{v}$, there must exist an $x \in \operatorname{Out}\left(H_{v}\right) \backslash U$, it follows that

$$
\left|V\left(H_{v}\right)\right|=\left|\operatorname{In}\left(H_{v}\right)\right|+\left|O u t\left(H_{v}\right)\right| \geq 1+d_{G}(x)-d_{G^{\prime}}(v) \geq \frac{n-\mu}{p}-d_{G^{\prime}}(v) .
$$

This proves Claim 2.

Claim 3 If $|U| \leq 1$, then $\left|W^{\prime}\right| \leq p$; if $|U| \geq 3$, then $\left|W^{\prime}\right| \leq p+1$.

Proof: Suppose first that $|U| \leq 1$. For any $v \in W^{\prime}$, by Claim 2 we have $\left|V\left(H_{v}\right)\right| \geq$ $\frac{n-\mu}{p}-d_{G^{\prime}}(v)$, so $n \geq\left|W^{\prime}\right|\left(\frac{n-\mu}{p}-c\right)$. This is equivalent to $\left|W^{\prime}\right| \leq \frac{n p}{n-\mu-p c}$. Since $\left|W^{\prime}\right|$ is an integer, we have $\left|W^{\prime}\right| \leq p$ when $n>p^{3}+6 p^{2}+5 p+\mu p+\mu$.

Suppose next that $|U| \geq 3$. So there must be exactly one vertex $u \in W^{\prime}$ such that $G[U] \subseteq H_{u}$. By Claim $2, n-1 \geq\left(\left|W^{\prime}\right|-1\right)\left(\frac{n-\mu}{p}-c\right)$. This is equivalent to $\left|W^{\prime}\right| \leq$ $\frac{(n-1) p}{n-\mu-p c}+1$. Since $\left|W^{\prime}\right|$ is an integer, we have $\left|W^{\prime}\right| \leq p+1$ when $n>p^{3}+6 p^{2}+4 p+\mu p+\mu$. 
Claim $4 V\left(G^{\prime}\right)=W$.

Proof: By contradiction, we assume that $V\left(G^{\prime}\right) \backslash W \neq \emptyset$. Note that every vertex in $V\left(G^{\prime}\right) \backslash W$ has degree at least $c+1$ in $G^{\prime}$. Since $G^{\prime}$ is simple $\left(G^{\prime}\right.$ is reduced), this means

$$
n^{\prime} \geq c+2
$$

Count the incidences to get $c\left|V\left(G^{\prime}\right) \backslash W\right| \leq 2\left|E\left(G^{\prime}\right)\right| \leq 4 n^{\prime}-8$, which means $\left|V\left(G^{\prime}\right) \backslash W\right| \leq$ $\frac{4 n^{\prime}-8}{c}$. It follows that

$$
|W|=n^{\prime}-\left|V\left(G^{\prime}\right) \backslash W\right| \geq\left(1-\frac{4}{c}\right) n^{\prime}+\frac{8}{c}
$$

By Claims 1 and 3, $|W| \leq\left|W^{\prime}\right|+1 \leq p+2$. Hence by (5.3) and (5.4),

$$
\left(1-\frac{4}{c}\right)(c+2)+\frac{8}{c} \leq\left(1-\frac{4}{c}\right) n^{\prime}+\frac{8}{c} \leq p+2
$$

It follows that $p+5=c \leq p+4$, a contradiction. Therefore, we must have $V\left(G^{\prime}\right)=W$.

Claim 5 Every vertex in $J_{p}(G)$ is contained in the pre-image of some vertex in $W^{\prime}$.

Proof: Since $n>p^{2}+6 p+\mu$, the degree of vertices in $J_{p}(G)$ will exceed $c$, and so Claim 5 follows from Claim 4.

Note that by Claim 5 and by Catlin's result, if $G^{\prime}$ has a $W^{\prime}$-eulerian subgraph, then $G$ has a $J_{p}(G)$-eulerian subgraph. Applying the discussion above, either $G^{\prime}$ has a $W^{\prime}$ eulerian subgraph, thereby $G$ has a $J_{p}(G)$-eulerian subgraph which is also a dominating eulerian subgraph and so $L(G)$ is hamiltonian, i.e., $L(G)$ has a 2-factor with only one component; or $G^{\prime}$ has no $W^{\prime}$-eulerian subgraph, since all vertices in $W^{\prime}$ are nontrivial, we can suppose that $W^{\prime}=\left\{v_{1}, v_{2}, \cdots, v_{m}\right\}$. For any $v_{i} \in W^{\prime}$, the pre-image of $v_{i}$ in $G$ denoted by $H_{i}$ is collapsible and hence has a spanning eulerian subgraph by Theorem 1.2.2. Moreover, $\left|V\left(H_{i}\right)\right| \geq 3$ since every vertex in $W^{\prime}$ is nontrivial. By Claim 1 , we divide 
$G^{\prime}$ into $m$ parts $P_{1}, P_{2}, \cdots, P_{m}$, where each $P_{i}$ is the union of $v_{i}$ and its neighbors from $W \backslash W^{\prime}$. Therefore, $G$ is divided into $m$ parts which are the corresponding pre-images of $P_{1}, P_{2}, \cdots, P_{m}$ and each part has a dominating eulerian subgraph $H_{i}$, and thus by $|U| \neq 2, \bigcup_{i=1}^{m} P_{i}$ is an $m$-system of $G$ that dominates. By Theorem 5.1.1, $L(G)$ has a 2 -factor with $m$ components. Since $m=\left|W^{\prime}\right| \leq p+1$ by Claim 3, it follows that $L(G)$ has a 2 -factor with at most $p+1$ components. Moreover, if $|U| \leq 1$, then $m=\left|W^{\prime}\right| \leq p$ by Claim 3, so $L(G)$ has a 2 -factor with at most $p$ components. Therefore, the proof of Theorem 5.1.7 is completed.

\subsection{Corollaries}

A graph is called $k$-supereulerian, if $G$ has a spanning even subgraph with at most $k$ components. The following result was proved recently.

Theorem 5.3.1 (Niu, Lai and Xiong, [40]) Let $G$ be a connected graph and $G^{\prime}$ be the reduction of $G$. Then $G$ is $k$-supereulerian if and only if $G^{\prime}$ is k-supereulerian.

By Theorem 5.3.1 and by the proof of Theorem 5.1.7, we obtain the following result.

Corollary 5.3.2 Let $G$ be a connected simple graph of order $n$, and let $p$ be a positive integer and $\mu$ a nonnegative integer. If $\max \{d(x), d(y)\} \geq \frac{n-\mu}{p}-1$ holds for any $x y \notin E(G)$, then for $n>p^{3}+6 p^{2}+6 p+\mu p+\mu, G$ is $(p+2)$-supereulerian, and $G$ is $(p+1)$-supereulerian if $|U| \leq 1$ where $U=\left\{v: d(v)<\frac{n-\mu}{p}-1\right\}$. And moreover $G$ is p-supereulerian if $U=\emptyset$.

It is not hard to get the following corollary by Theorem 2.1 of Lai [37].

Corollary 5.3.3 Let $G$ be a simple graph with $\kappa^{\prime}\left(G-D_{1}(G)\right) \geq 2$ and with $n$ vertices. If $\max \{d(x), d(y)\} \geq n / 5-1$ holds for every edge $x y \in E(G)$, then for $n$ large, $L(G)$ has a 2-factor with at most 3 components. 
Proof. Let $K_{2,3}, K_{2,5}, W_{3}^{\prime}, W_{4}^{\prime}, L_{1}, L_{2}$ and $L_{3}$ be the graphs defined in Figures 1,2 and 3 , and let $F=\left\{K_{2,3}, K_{2,5}, W_{3}^{\prime}, W_{4}^{\prime}, L_{1}, L_{2}, L_{3}\right\}$. Using the labels in Figures 1, 2 and 3, for each $L \in F$, we define $B(L)$, the bad set of $L$, to be the vertex subset of $V(L)$ that are labelled with the $b_{i}$ 's. By Theorem 2.1 of Lai [37], one of the following must hold:

(i) $L(G)$ is hamiltonian, or

(ii) $G$ can be contracted to a member $L \in F$ such that the pre-image of every vertex in $B(L)$ intersects $J$.

Note that $V(G)-J_{5}(G)$ is an independent set of $G$. Hence any eulerian subgraph $H$ of $G$ with $J \subseteq V(H)$ must be a dominating eulerian subgraph of $G$. Observing all the graphs in the following figures, we can see that there are at most 3 components of dominating eulerian subgraphs in $G$. So $L(G)$ has a 2-factor with at most 3 components.
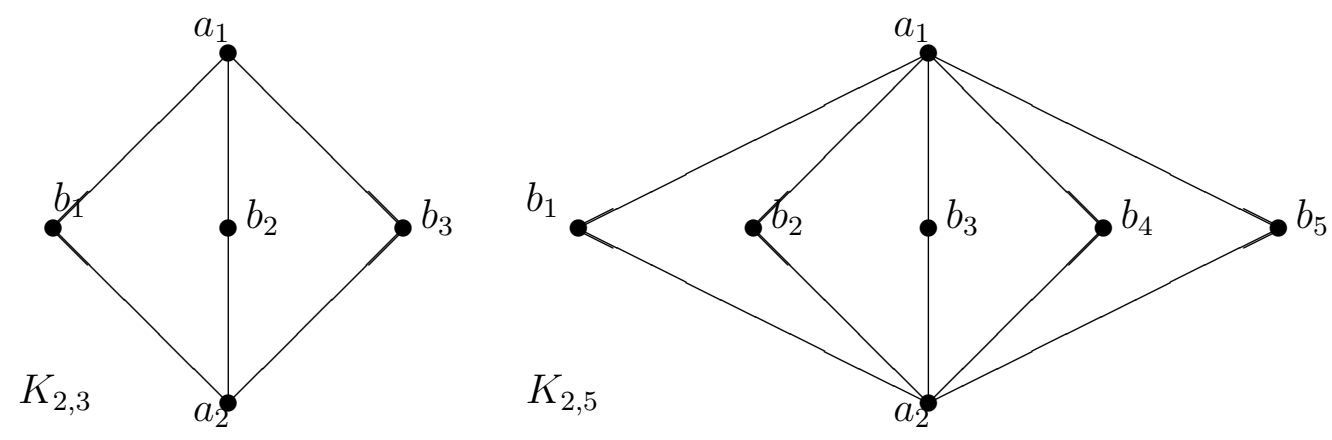

Figure 1: The graphs $K_{2,3}$ and $K_{2,5}$
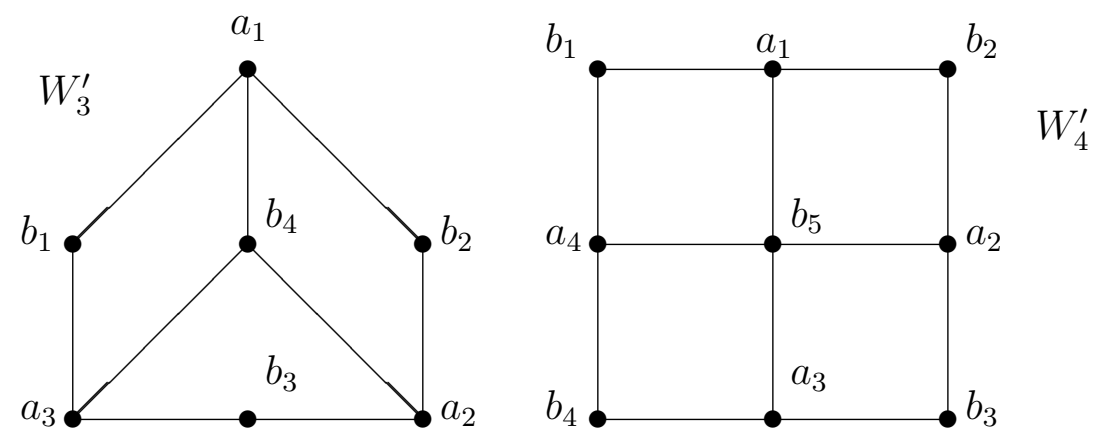

Figure 2: The graphs $W_{3}^{\prime}$ and $W_{4}^{\prime}$ 

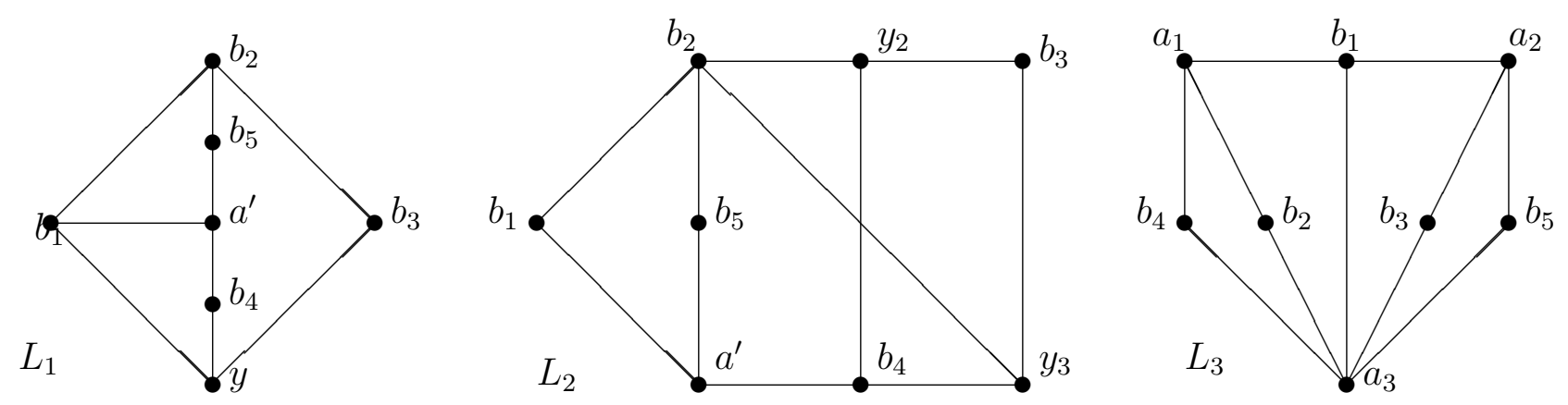

Figure 3: The graphs $L_{1}, L_{2}$ and $L_{3}$

\section{Sharpness}

In this part, we shall give an example to show the sharpness of Theorem 5.1.7 and Corollary 5.3.2. Let $G_{1}, G_{2}, \cdots, G_{p}$ be $p$ vertex-disjoint complete graphs of order $\frac{n-\mu}{p}$ and $G_{\mu}$ an additional graph of order $\mu$. Obtain $G$ by joining exactly one vertex of $G_{i}$ to exactly one vertex of $G_{\mu}$ for $i=1,2, \cdots, p$. Note that $\max \{d(x), d(y)\} \geq \frac{n-\mu}{p}-1$ for each $x y \notin E(G)$ (where $\mu=\left|\left\{v: d(v)<\frac{n-\mu}{p}-1\right\}\right|$ ) and the equation can be achieved for some pairs of nonadjacent vertices $x, y$. If $\mu>2$ and $n$ is sufficiently large, then $L(G)$ has a 2-factor with $p+1$ components; if $\mu=1$, then $L(G)$ has a 2-factor with $p$ components; if $\mu=2$, then $L(G)$ has no 2-factor at all. For $\mu=0$, let $G^{\prime}$ be the graph of order $n$ obtained from $G_{1}, G_{2}, \cdots, G_{p}$ such that $G /\left\{G_{1}, G_{2}, \cdots, G_{p}\right\}$ is a tree. Then $G^{\prime}$ satisfies the condition of Theorem 5.1.7 and $L\left(G^{\prime}\right)$ has a 2-factor with $p$ components. This shows Theorem 5.1.7 is best possible.

If $\mu=2, G$ is $(p+2)$-supereulerian but not $(p+1)$-supereulerian; if $\mu \neq 2$, then $G$ is $(p+1)$-supereulerian but not $p$-supereulerian; if $\mu=0$ then $G^{\prime}$ is $p$-supereulerian but not $(p-1)$-supereulerian. This shows that Corollary 5.3.2 is best possible. 


\section{Chapter 6}

\section{Inverse problems on Graphs}

\subsection{Introduction}

In some applications involving a network, it is needed to recover the inner structure of the network from measurements collected at the boundary of the network, analogous to problems arising from tomography. We study a particular type of such problems, which can be considered as a discrete version of the well-known inverse conductivity problem in continuous media. In this setting, the network is represented by a connected graph with a weight distribution on the edges (a weighted graph), and a subset of nodes is designated as the "boundary" of the network. Then there is a function defined on the graph, representing a physical variable such as potentials on the nodes, and it is assumed that this function satisfies an equation on the graph that is analogous to the Laplace equation that governs the potential on continuous media. The inverse problem is the recovery of the network structure from certain knowledge of the solution to the Laplace equation. In order to understand the difficulty and aspects of the problem that are unique in the discrete setting, we will focus on the recovery of the weight distribution on some specific graph structures.

Let $G(V, E)$ be an undirected graph. A weight function (distribution) $\omega(x, y)$ is defined as a map $\omega: V \times V \rightarrow[0, \infty)$ that satisfies 
(i) $\omega(x, x)=0$ for $x \in V$;

(ii) $\omega(x, y)=\omega(y, x)$ if $x \sim y(x$ and $y$ are connected by an edge in $E)$;

(iii) $\omega(x, y)=0$ if and only if $x \not y$.

A graph $G(V, E)$ with a weight function $\omega$ is called a weighted graph, denoted by $G(V, E ; \omega)$. The degree of a vertex $x$ in $G(V, E ; \omega)$ is defined as $d_{\omega} x=\sum_{y \in V} \omega(x, y)$. For a subgraph $S$ of $G=G(V, E ; \omega)$, the boundary $\partial S$ of $S$ is the set of all vertices $z \in V$ not in $S$ but adjacent to some vertex in $S$, i.e., $\partial S=\{z \in V-S \mid z \sim y$ for some $y \in S\}$. Let $f$ be a real-valued function defined on $V$. An operator on $f$ defined by

$$
\Delta_{\omega} f(x)=\sum_{y \in V}[f(y)-f(x)] \cdot \frac{\omega(x, y)}{d_{\omega} x}, \quad \forall x \in V
$$

is called the $\omega$-Laplacian. The $\omega$-Laplacian equation refers to the equation $\Delta_{\omega} f(x)=0$ for $x \in V$, and solutions to this equation are called $\omega$-harmonic functions on $V$. On $\partial S$, define the (outward) normal derivative of $f$ as

$$
\frac{\partial f}{\partial_{\omega} n}(z)=\sum_{y \in S}[f(z)-f(y)] \cdot \frac{\omega(z, y)}{d_{\omega}^{\prime} z}, \quad \forall z \in \partial S,
$$

where $d_{\omega}^{\prime} z=\sum_{y \in S} \omega(z, y)$.

Given $G(V, E ; \omega), S \subset V$ and a function $\psi$ on $\partial S$, the Neumann Boundary Value Problem (NBVP) is to find a function $f$ on $V$ that satisfies

$$
\left\{\begin{array}{rlrl}
\Delta_{\omega} f(x) & =0, & & x \in S \\
\frac{\partial f}{\partial_{\omega} n}(z)=\psi(z), & z \in \partial S
\end{array}\right.
$$

The inverse problem related to (6.1) that we intend to study is as follows. If we are given the values of $f$ and $\frac{\partial f}{\partial \omega n}$ on $\partial S$, is it possible to recover the weight function $\omega$ on $S$, provided that $G(V, E)$ is also known? In 2005, Chung and Berenstein showed the following global uniqueness result for the inverse conductivity problem in a network. 
Theorem 6.1.1 (Chung and Berenstein, [20]) Let $\omega_{1}$ and $\omega_{2}$ be weights with $\omega_{1} \leq \omega_{2}$ on $\bar{S} \times \bar{S}$ and $f_{1}, f_{2}: \bar{S} \rightarrow \mathbb{R}$ be functions satisfying, for $j=1,2$,

$$
\left\{\begin{array}{l}
\Delta_{\omega_{j}} f_{j}(x)=0, \quad x \in S \\
\frac{\partial f}{\partial_{\omega_{j}} n}(z)=\psi(z), \quad z \in \partial S \\
\int_{S} f_{j} d_{\omega_{j}}=K
\end{array}\right.
$$

for a given function $\psi: \partial S \rightarrow \mathbb{R}$ with $\int_{\partial S}=0$ and a given constant $K>0$. If we assume that

(i) $\omega_{1}(z, y)=\omega_{2}(z, y)$ on $\partial S \times S$,

(ii) $f_{1}\left|\partial S=f_{2}\right| \partial S$,

then we have $f_{1}=f_{2}$ on $\bar{S}$ and $\omega_{1}=\omega_{2}$ on $\bar{S} \times \bar{S}$.

To relax the monotonicity condition for $\omega$, we study the case when $S$ is the vertex set of a path (a path is a simple tree) with $n$ vertices in the following section.

\subsection{NBVP on a path}

We consider the NBVP

$$
(*) \quad(N B V P)\left\{\begin{array}{lc}
\Delta_{\omega} f(x)=0, & x \in S \\
\frac{\partial f}{\partial_{\omega} n}(z)=\psi(z), & z \in \partial S
\end{array}\right.
$$

where $S$ is the vertex set of a path with $n$ vertices denoted by natural numbers $\{1,2,3, \cdots, n\}$ and where $i$ is adjacent to $i+1$ for $1 \leq i \leq n-1$ and $\partial S$ contains two vertices $\{0, n+1\}$ and 0 is adjacent to $1, n+1$ is adjacent to $n$. As [20] remarked, the solution to the NBVP is uniquely determined by the Neumann data $\psi$ on $\partial S$ up to an additive constant. Thus we add the condition $\int_{\partial S} f=c$ (a given constant). However, the solvability of the solution to the NBVP on a general network is not known yet. In this section we are going to show the following theorem.

Theorem 6.2.1 The weight distributions on a path can be uniquely solved by the boundary values of both $f$ and $\frac{\partial f}{\partial_{\omega} n}$ 
Proof. For the weight function $\omega(x, y)$, we use $\omega_{i}$ to denote $\omega(i, i+1)$. We can rewrite (*) as a system of equations $A \vec{x}=\vec{v}$, where $A$ is a tridiagonal matrix as follows

$$
\left(\begin{array}{ccccccccc}
1 & -1 & 0 & 0 & 0 & \cdots & 0 & 0 & 0 \\
\frac{\omega_{0}}{\omega_{0}+\omega_{1}} & -1 & \frac{\omega_{1}}{\omega_{0}+\omega_{1}} & 0 & 0 & \cdots & 0 & 0 & 0 \\
0 & \frac{\omega_{1}}{\omega_{1}+\omega_{2}} & -1 & \frac{\omega_{2}}{\omega_{1}+\omega_{2}} & 0 & \cdots & 0 & 0 & 0 \\
0 & 0 & 0 & 0 & 0 & \cdots & \frac{\omega_{n-1}}{\omega_{n-1}+\omega_{n}} & -1 & \frac{\omega_{n}}{\omega_{n-1}+\omega_{n}} \\
0 & 0 & 0 & 0 & 0 & \cdots & 0 & -1 & 1
\end{array}\right)
$$

$\vec{x}$ is a column vector as follows

$$
\left(\begin{array}{llllll}
f(0) & f(1) & f(2) & \ldots & f(n) & f(n+1)
\end{array}\right)^{T}
$$

and $\vec{v}$ is a constant column vector as follows

$$
\left(\begin{array}{llllll}
\psi(0) & 0 & 0 & \ldots & \psi(n) & \psi(n+1)
\end{array}\right)^{T} .
$$

Notice that the boundary conditions are given, so the following are known: $f(0), f(n+1)$, $\omega_{0}, \omega_{n}$. By the Green's formula

$$
\int_{S} \Delta_{\omega} f=\int_{\partial S} \frac{\partial f}{\partial_{\omega} n},
$$

it is necessary that $\int_{\partial S} \psi=0$, i.e., $\psi(0) \omega_{0}+\psi(n+1) \omega_{n}=0$. So $\psi(0) \omega_{0}=-\psi(n+1) \omega_{n}$. Let $B=\left(\begin{array}{ll}A & \vec{v}\end{array}\right)$. By taking elementary row operations on the matrix $B$, we obtain an upper triangular matrix $B^{\prime}$ as follows

$$
\left(\begin{array}{cccccccccc}
1 & -1 & 0 & 0 & 0 & \cdots & 0 & 0 & 0 & \psi(0) \\
0 & -1 & 1 & 0 & 0 & \cdots & 0 & 0 & 0 & -\frac{\omega_{0} \psi(0)}{\omega_{1}} \\
0 & 0 & -1 & 1 & 0 & \cdots & 0 & 0 & 0 & -\frac{\omega_{0} \psi(0)}{\omega_{2}} \\
& & & \cdots & \cdots & \cdots & & & & \\
0 & 0 & 0 & 0 & 0 & \cdots & 0 & -1 & 1 & -\frac{\omega_{0} \psi(0)}{\omega_{n}} \\
0 & 0 & 0 & 0 & 0 & \cdots & 0 & 0 & 0 & 0
\end{array}\right)
$$

It is obvious to see the rank of matrix $A$ is $n+1$. Since $\int_{\partial S} f=c$, we get one more equation $\left(\omega_{0}+\omega_{1}\right) f(1)+\left(\omega_{1}+\omega_{2}\right) f(2)+\cdots+\left(\omega_{n-2}+\omega_{n-1}\right) f(n-1)+\left(\omega_{n-1}+\omega_{n}\right) f(n)=c$ and the corresponding vector is

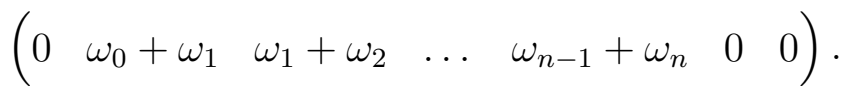


Now we replace the last row (0 vector) of matrix $B^{\prime}$ by the above vector, then the new matrix has full rank $n+2$ and correspondingly the system of linear equations has a unique solution. Therefore the proof is done.

\subsection{Inverse problems on electrical networks}

For the electrical network $S$, the function $\gamma$ defined on the edges of $S$ is called its conductivity. The network is considered $\gamma$-harmonic if for each node in $S$, the sum of currents out of that node is 0 . We use $f(x)$ to denote the potential on $x$ and define the Laplace operator as $\Delta_{\gamma} f(x)=\sum_{y \in \bar{S}}[f(x)-f(y)] \gamma(x, y), \forall x \in S$. The Kirchhoff Matrix $\mathbf{K}$ takes potentials on the network to currents on the network, and is defined by:

$$
K(i, j)= \begin{cases}-\gamma(i, j), & 1 \leq i<j \leq n \\ \sum_{i \neq j} \gamma(i, j), & 1 \leq i=j \leq n\end{cases}
$$

The Kirchhoff matrix can be block partitioned in the following manner:

$$
K=\left(\begin{array}{cc}
A & B \\
B^{T} & C
\end{array}\right)
$$

where $A$ corresponds to all boundary nodes and $C$ corresponds to all internal nodes. From the block structure of the Kirchhoff matrix we can derive the Dirichlet-to-Neumann response matrix $\Lambda=A-B C^{-1} B^{T}$, which takes potentials at the boundary of $\Lambda$ to currents through the boundary nodes of $S$.

\subsubsection{Network on a complete graph}

A graph $G$ is said to be complete if there is an edge between every pair of nodes, representing a network that every node is connected to every other node within the network. The quality of the connection between two nodes will be represented by a conductivity value on the edge between the two nodes. Let $\{1,2, \cdots, n\}$ denote the $n$ nodes $(n \geq 4)$, and $\left\{\gamma_{i j}\right\}_{1 \leq i<j \leq n}$ denote the conductivity distribution on the edges, and we assume that 
these are normalized conductivities and there is no false connection on the network:

$$
\sum_{1 \leq i<j \leq n} \gamma_{i j}=1 \quad \text { and } \quad \gamma_{j i}=\gamma_{i j}>0
$$

These $n(n-1) / 2$ conductivities are designated as descriptors of the network structure. If we let $\left\{u_{i}\right\}_{i=1}^{n}$ denote the potentials on the nodes, then on each "interior" node there holds the discrete Laplace equation, which simply states that the net current out of the node is zero:

$$
\sum_{j \neq i} \gamma_{i j}\left(u_{i}-u_{j}\right)=0
$$

Consider the following detection scenario: we designate a subset of nodes, say $\{1,2, \cdots, b\}$, as "boundary" nodes, representing the use of these nodes as probes. The remaining nodes are kept as interior nodes. If a node is chosen to be a boundary node, then it connects to only interior nodes in the network and the edges from this node to other boundary nodes are removed, which can be viewed as setting the conductivity to be zero on these edges. In this case there are $e \equiv b(n-b)+(n-b)(n-b-1) / 2=(n-b)(n+b-1) / 2$ many edges and thus these many conductivities $\left\{\gamma_{i j}\right\}$. At each boundary node $k(1 \leq k \leq b)$, we assume that we can apply a potential $u_{k}$ and measure the induced net current $I_{k}$ out of the node:

$$
I_{k}=\sum_{j=b+1}^{n} \gamma_{k j}\left(u_{k}-u_{j}\right) .
$$

Thus, at each node, there is an equation for the current out of the node, either by (6.3) for boundary node or by (6.2) for interior node, and these $n$ equations can be written in the matrix form as $\mathbf{K} \vec{u}=\vec{I}$ from node potentials $\vec{u}=\left[u_{j}\right]^{T}$ to node currents $\vec{I}=\left[I_{j}\right]^{T}$. The matrix $\mathbf{K}=\left[K_{i j}\right]_{n \times n}$ is known as the Kirchhoff matrix that represents the linear relation from potentials to currents in a general network, where we have taken the liberty to assume that $\gamma_{i j}=0$ when $1 \leq i<j \leq b$ for our situation.

Proposition 6.3.1 The $n \times n$ Kirchhoff matrix $\mathbf{K}$ is positive semi-definite with rank $n-1$.

Proof. It is known that the Kirchhoff matrix has a factorization $\mathbf{K}=Q Q^{T}$ (see e.g. [23]) where $Q$ is an $n \times e$ matrix, whose $k$-th column corresponding to the $k$-th edge that 
connects nodes $i$ and $j(i<j)$ is given by $q_{i k}=\sqrt{\gamma_{i j}}, q_{j k}=-\sqrt{\gamma_{i j}}$, and $q_{l k}=0$ for all $l \neq i, j$. Clearly $\vec{u}^{T} \mathbf{K} \vec{u}=\left\|Q^{T} \vec{u}\right\|^{2} \geq 0$ for any vector $\vec{u}$, hence $\mathbf{K}$ is positive semidefinite. If $\mathbf{K} \vec{u}=\overrightarrow{0}$, then $Q^{T} \vec{u}=\overrightarrow{0}$, hence for each pair of adjacent nodes $i$ and $j$, we have $u_{i}=u_{j}$. Since each node is connected to at least another node, all $u_{j}$ 's are equal. Therefore we see that the null-space of $\mathbf{K}$ is the one-dimensional subspace spanned by the vector $[1, \cdots, 1]^{T}$. Hence the rank of $\mathbf{K}$ is $n-1$.

If we partition the potentials and currents according to boundary and interior nodes in our setting:

$$
\vec{u}=\left[\begin{array}{c}
\vec{u}_{B} \\
\vec{u}_{I}
\end{array}\right]=\left[\begin{array}{c}
u_{1} \\
\vdots \\
u_{b} \\
\hline u_{b+1} \\
\vdots \\
u_{n}
\end{array}\right] \text { and } \vec{I}=\left[\begin{array}{c}
\vec{I}_{B} \\
\vec{O}
\end{array}\right]=\left[\begin{array}{c}
I_{1} \\
\vdots \\
I_{b} \\
\hline 0 \\
\vdots \\
0
\end{array}\right]
$$

then the Kirchhoff matrix $\mathbf{K}$ is naturally partitioned into blocks as

$$
\mathbf{K}=\left[\begin{array}{c|c}
D & B \\
\hline B^{T} & C
\end{array}\right],
$$

where $D$ particularly is the diagonal matrix corresponding to the disconnectedness between boundary nodes, with $k$-th diagonal entry as $\sum_{j=b+1}^{n} \gamma_{k j}(1 \leq k \leq b)$. Note that $C$ is diagonally dominant hence it is nonsingular.

In our study we are interested in the map from the boundary potentials $\vec{u}_{B}$ to the induced boundary currents $\vec{I}_{B}$, which is often regarded as the Dirichlet-to-Neumann map in the literature. From the block structure (6.4) of the Kirchhoff matrix, by eliminating $\vec{u}_{I}$, we can easily obtain this Dirichlet-to-Neumann response matrix as

$$
\mathbf{K}_{b}=D-B C^{-1} B^{T},
$$

and the relation between boundary potentials and currents is simply $\mathbf{K}_{b} \vec{u}_{B}=\vec{I}_{B}$. The matrix $\mathbf{K}_{b}$ shares similar properties of the Kirchhoff matrix, as stated below. 
Proposition 6.3.2 The $b \times b$ Dirichlet-to-Neumann matrix $\mathbf{K}_{b}$ is positive semi-definite with rank $b-1$.

Proof. For any $b$-vector $\vec{x}$, let $\vec{y}=-C^{-1} B^{T} \vec{x}$ and $\vec{u}=\left[\begin{array}{l}\vec{x} \\ \vec{y}\end{array}\right]$. Then it is straightforward to verify that

$$
\vec{x}^{T} \mathbf{K}_{b} \vec{x}=\vec{u}^{T} \mathbf{K} \vec{u} \quad \text { and } \quad \mathbf{K}_{b} \vec{x}=\overrightarrow{0} \quad \Longleftrightarrow \quad \mathbf{K} \vec{u}=\overrightarrow{0} .
$$

Therefore, the positive semi-definiteness of $\mathbf{K}_{b}$ comes from that of $\mathbf{K}$, and null-space of $\mathbf{K}_{b}$ and that of $\mathbf{K}$ must have the same dimension, which for $\mathbf{K}$ is 1 . Hence the rank of $\mathbf{K}_{b}$ is $b-1$.

Our goal now is to determine for what $b$ values it is possible to find the conductivities $\left\{\gamma_{i j}\right\}$ from boundary current measurements $\left\{I_{i}\right\}$ induced by properly chosen boundary potentials $\left\{u_{i}\right\}_{i=1}^{b}$. So far we can only deal with one extreme case: $b=n-1$ (only one interior node).

Lemma 6.3.3 When $b=n-1$ (only node $n$ is interior), the $n-1$ conductivities $\left\{\gamma_{i n}\right\}_{i=1}^{n-1}$ can be found from only one set of current measurements.

Proof. For simplicity in the following, we denote $\gamma_{k}=\gamma_{k n}$ for $k=1, \cdots, n-1$ (hence $\left.\sum_{k=1}^{n-1} \gamma_{k}=1\right)$. From (6.5), we have

$$
\mathbf{K}_{n-1}=\left[\begin{array}{cccc}
\gamma_{1} & & & \\
& \gamma_{2} & & \\
& & \ddots & \\
& & & \gamma_{n-1}
\end{array}\right]-\left[\begin{array}{c}
\gamma_{1} \\
\gamma_{2} \\
\vdots \\
\gamma_{n-1}
\end{array}\right]\left[\begin{array}{llll}
\gamma_{1} & \gamma_{2} & \cdots & \gamma_{n-1}
\end{array}\right] .
$$

To find the conductivities, we choose the applied boundary potentials as $\left[u_{1}, u_{2}, \cdots, u_{n-1}\right]=$ $[1,0, \cdots, 0]$, and let its induced currents be $\left[I_{1}, I_{2}, \cdots, I_{n-1}\right]$. The potential at the only interior node $n$ is $u_{n}=\gamma_{1}$ (since $\left.\sum \gamma_{k}=1\right)$. Therefore we have $\gamma_{1}\left(1-\gamma_{1}\right)=I_{1}>0$ and $-\gamma_{j} \gamma_{1}=I_{j}<0$ for $j=2, \cdots, n-1$. Hence $I_{1} \leq \frac{1}{4}$ and $\gamma_{1}=\frac{1}{2}-\sqrt{\frac{1}{4}-I_{1}}$ and $\gamma_{j}=\frac{-I_{j}}{I_{1}}\left(\frac{1}{2}+\sqrt{\frac{1}{4}-I_{1}}\right)$ for $j=2, \cdots, n-1$. That is, one set of current measurements is sufficient to determine the network conductivity distribution. 
Above all, when the network structure is a star $K_{1, n-1}$ (the case for $b=n-1$ ), the conductivity distribution can be uniquely determined.

\subsubsection{Network on a tree}

A tree is a connected graph without cycles, and so a star is also a tree. The only interior node of a star is called the center of the star. Any boundary node adjacent to the center is called a leaf node. To generalize the result in section 6.3.1, we firstly consider the tree network $G$ by connecting the centers of two stars $K_{1, n-1}$ and $K_{1, m-1}$ by one edge.

Claim 6 The conductivity distribution on $G$ can be uniquely determined.

Proof. We contract one star $K_{1, n-1}$ of $G$ to a node and this node is still adjacent to the center of the other star $K_{1, m-1}$. Hence, they will form a new star $K_{1, m}$. By Lemma 6.3.3, we can determine the conductivity distribution of $K_{1, m}$. Symmetrically, we also can contract $K_{1, m-1}$ and determine the conductivity distribution on the new star $K_{1, n}$. Then the only undetermined parameter left is the conductivity between the two centers in $G$. Since the potential and current on any leaf node can be measured and the conductivity between the leaf node and its corresponding center is also determined, it is easy to calculate the potential on any center. We pick up one center $u$. Since $u$ is an interior node, the currents flowing-in and flowing-out on $u$ are the same. Noticing that the currents on all leaf nodes of $u$ are measured, it is easy to determine the current between $u$ and the other center node $v$. Since the potentials on both $u$ and $v$ can be calculated, it follows that we can determine the conductivity between them. Above all, the conductivity distribution on $G$ can be uniquely determined.

Now we study the more general case. Let $u_{1}, u_{2}, \cdots, u_{n}$ be the centers of $n$ stars $K_{1, m_{1}-1}, K_{1, m_{2}-1}, \cdots, K_{1, m_{n}-1}$. Suppose $G$ is a connected graph and formed by connecting only these centers of stars with edges such that $G$ has no cycles, i.e., $G$ is a tree.

Theorem 6.3.4 The conductivity distribution on the network $G$ can be uniquely determined. 
Proof. Let $T$ be the tree spanned by $u_{1}, u_{2}, \cdots, u_{n}$. By Claim 6 , we can determine the conductivities on each star in graph $G$. So far, the only problem left is to determine the conductivity on the tree $T$. Since each $u_{i}$ is a center of a star, it is easy to calculate its potential by the information on any one of its leaf neighbor. In the tree $T$, we pick up each leaf node $u_{i}$ and its neighbor node $u_{j}$. Since the current flowing out from $u_{i}$ to $u_{j}$ can be calculated by the information on $u_{i}$ 's leaf nodes in $G$ and the potentials on both $u_{i}$ and $u_{j}$ are already known, we can uniquely determine the conductivity between $u_{i}$ and $u_{j}$. If we keep calculating the conductivity between two nodes according to the direction from leaf nodes to the root node, then we will uniquely determine the conductivities on all edges of the tree $T$.

Theorem 6.3.5 The conductivity distribution on the network $G$ is not unique if $G$ is not a tree.

Proof. To consider the easiest case, we assume that $G$ is a cycle with only three nodes $u_{1}, u_{2}$ and $u_{3}$. As we know, it is easy to calculate the potentials on these three nodes and the current flowing out from each one to its neighbors. We use $v_{1}, v_{2}$ and $v_{3}$ to denote potentials on them respectively, and use $I_{1}, I_{2}$ and $I_{3}$ to denote currents flowing out of them respectively. As before, we still use $\gamma_{i, j}$ to denote the conductivity between $u_{i}$ and $u_{j}$. Then we have the following system of equations:

$$
\left\{\begin{array}{l}
\left(v_{1}-v_{2}\right) \gamma_{1,2}+\left(v_{1}-v_{3}\right) \gamma_{1,3}=I_{1} \\
\left(v_{2}-v_{1}\right) \gamma_{1,2}+\left(v_{2}-v_{3}\right) \gamma_{2,3}=I_{2} \\
\left(v_{3}-v_{1}\right) \gamma_{1,3}+\left(v_{3}-v_{2}\right) \gamma_{2,3}=I_{3}
\end{array}\right.
$$

The three equations are linearly dependent since $I_{1}+I_{2}+I_{3}=0$. So there are infinite number of solutions for $\gamma_{i, j}$, i.e., the conductivity on a cycle can not be uniquely determined. 


\section{Bibliography}

[1] A. Ainouche, A common generalization of Chvátal-Erdös and Fraisse's sufficient conditions for hamiltonian graphs, Discrete Math. 142 (1995) 1-19.

[2] J. C. Benhocine and J. L. Fouquet, The Chvátal-Erdös condition and pancyclic line graphs, Discrete Math. 66 (1987) 21-26.

[3] J. A. Bondy and U. S. R. Murty, "Graph Theory with Applications". American Elsevier (1976).

[4] J. Brousek, Z. Ryjáček and O. Favaron, Forbidden subgraphs, hamiltonianicity and closure in claw-free graph, Discrete Math. 196 (1999) 29-50.

[5] P. A. Catlin, Edge-connectivity and edge-disjoint spaning trees, Ars Combinatoria, accepted.

[6] P. A. Catlin, Supereulerian graph, collapsible graphs and 4-cycles, Congressus Numerantium 56 (1987) 223-246.

[7] P. A. Catlin, A reduction method to find spanning eulerian subgraphs, J. Graph Theory 12 (1988) 29-44.

[8] P. A. Catlin, Contractions of graphs with no spanning Eulerian subgraphs. Combinatorica 8 (1988) 313-321.

[9] P. A. Catlin, The reduction of graph families closed under contraction, Discrete Math. 160 (1996), 67-80. 
[10] P. A. Catlin, Z. Y. Han and H.-J. Lai, Graphs without spanning closed trails, Discrete Mathematics 160 (1996) 81-91.

[11] P. A. Catlin, A. M. Hobbs and H.-J. Lai, Operations and graph families, Discrete Math, 230 (2001) 71-98.

[12] P. A. Catlin and H.-J. Lai, Spanning trails joining two given edges, "Graph theory, combinatorics, and applications", eds. by Y. Alavi, G. Chartrand, O. R. Ollermann, A. J. Schwenk, John Wiley and Sons, Inc. (1991) 207-222.

[13] P. A. Catlin, T. N. Janakiraman and N. Srinivasan, Hamilton cycles and closed trails in iterated line graphs, J. Graph Theory, 3 (1990) 347-364.

[14] G. Chartrand, On Hamiltonian line-graphs, Trans. Amer. Math. Soc. 134 (1968) 559-566.

[15] G. Chartrand and C. E. Wall, On the hamiltonian index of a graph, Studia Sci. Math. Hungar. 8 (1973) 43-48.

[16] Z.-H. Chen, Reduction of Graphs and Spanning Eulerian Subgraphs, Ph. D dissertation, Wayne State University, (1991).

[17] Z.-H Chen, Supereulerian graphs and the Petersen graph, J. of Comb. Math. and Comb. Computing 9 (1991) 70-89.

[18] Z.-H Chen, Supereulerian graphs, independent set, and degree-sum conditions, Discrete Math. 179 (1998) 73-87.

[19] G. Chen, J. R. Faudree, R. J. Gould, A. Saito, 2-Factors in claw-free graphs. Discuss. Math. Graph Theory 20 (2000) 165-172.

[20] S.-Y. Chung and C. A. Berenstein, $\omega$-harmonic functions and inverse conductivity problems on networks, SIAM J. APPL. MATH, 65(4), 2005, pp. 1200-1226.

[21] V. Chvátal and P. Erdös, A note on Hamiltonian circuits, Discrete Math. 2 (1972) 111-113. 
[22] L. H. Clark and N. C. Wormald, Hamiltonian-like indices of graphs, Ars Combinatoria, 15 (1983) 131-148.

[23] E. B. Curtis, D. Ingerman, and J. A. Morrow, Circlular planar graphs and resistor networks, Linear Alg. Appl. 283 (1998) 115-150.

[24] H. Enomoto, A. Kaneko, A. Saito and B. Wei, Long cycles in triangle-free graphs with prescribed independence number and connectivity, Journal of Combinatorial Theory, Series B 91 (2004) 43-55.

[25] J. R. Faudree, R. J. Gould, M. S. Jacobson, L. Lesniak, A. Saito, Toughness, degrees and 2-factors. Discrete Math. 286 (2004) 245-249.

[26] J. Fujisawa, L. Xiong, K. Yoshimoto, S. Zhang, The upper bound of the number of cycles in a 2-factor of a line graph. J. Graph Theory 55 (2007) 72-82

[27] R. Gould and E. Hynds, A note on cycles in 2-factor of line graphs, Bull. of ICA. 26 (1999) 46-48.

[28] R. Häggkvist and C. Thomassen, Circuits through specified edges, Discrete Math. 41 (1982) 29-34

[29] F. Harary and C. St. J. A. Nash-Williams, On eulerian and hamiltonian graphs and line graphs, Can. Math. Bull. 8 (1965) 701-710.

[30] J. van den Heuvel, Extensions and consequences of Chvátal-Erdös' theorem, Graphs and Combinatorics 12 (1996) 231-237.

[31] B. Jackson and O. Ordaz, Chvátal-Erdös conditions for paths and cycles in graphs and digraphs. A survey, Discrete Math. 84 (1990) 241-254.

[32] F. Jaeger, A note on subeulerian graphs, J. Graph Theory. 3 (1979) 91-93.

[33] A. Kanneko and Y. Yoshimoto, A 2-factor with two componencts of a graph satisfying the Chvátal-Erdös condition, J. Graph Theory. 43 (2003) 269-279.

[34] H.-J. Lai, On the hamiltonian index, Discrete Math. 69 (1988) 43-53.

[35] H.-J. Lai, Reduced graphs of diameter two, J. Graph Theory. 14 (1990) 77-87. 
[36] H.-J. Lai, Graphs whose edges are in small cycles, Discrete Math. 94 (1991) 11-22.

[37] H.-J. Lai, Eulerian subgraphs containing given vertices and hamiltonian line graphs. Discrete Math. 178 (1998) 93-107.

[38] T. Euczak and F. Pfender, Claw-free 3-connected $P_{11}$-free graphs are hamiltonian, J. Graph Theory. 47 (2004) 111-121.

[39] M. M. Matthews and D. P. Sumner, Hamiltonian results in $K_{1,3}$-free graphs, J. Graph Theory. 8 (1984) 139-146.

[40] Z. Niu, H.-J. Lai and L. Xiong, Spanning subgraph with eulerian components Preprint.

[41] Z. Niu, L. Xiong, On the bounded number of components in even factor. (preprint)

[42] Z. Ryjáček, On a closure concept in claw-free graphs, J. Combin. Theory Ser. B 70 (1997) 217-224.

[43] M. L. Saražin, A simple upper bound for the hamiltonian index of a graph, Discrete Math. 134 (1994) 85-91.

[44] Y. Shao, Claw-free graphs and line graphs, Dissertation for Ph.D, Department of Mathematics, West Virginia University, 2005.

[45] C. Thomassen, Reflections on graph theory, J. Graph Theory. 10 (1986) 309-324.

[46] B. Wei and Y.J. Zhu, The Chvátal-Erdös conditions for panconnectivity of trianglefree graphs, Discrete Math. 252 (2002) 203-214.

[47] L. Xiong and Z. Liu, Hamiltonian iterated line graphs, Discrete Math. 256 (2002) 407-422.

[48] L. Xiong, H.J. Broersma, X. Li and M. Li, The hamiltonian index of a graph and its branch-bonds, Discrete Math. 285 (2004) 279-288.

[49] L. Xiong and Q. Wu, The hamiltonian index of a 2-connected graph, Discrete Math.(2008), doi:10.1016/j.disc.2007.12.008. 